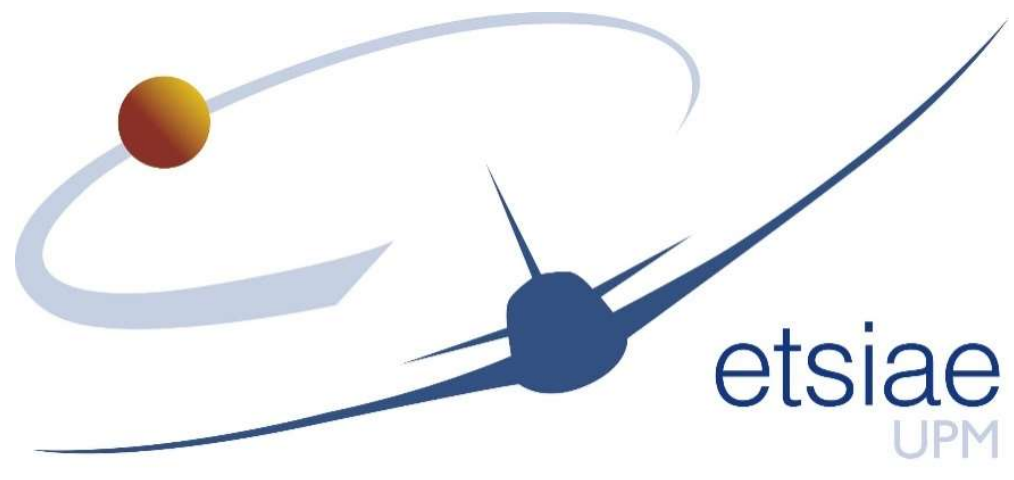

UNIVERSIDAD POLITÉCNICA DE MADRID

ESCUELA TÉCNICA SUPERIOR DE INGENIERIA AERONÁUTICA Y DEL ESPACIO

\title{
THEORETICAL MODEL FOR DROPLET DEFORMATION AND TRAJECTORY IN CONTINOUSLY ACCELERATING FLOWS
}

TESIS DOCTORAL

SUTHYVANN SOR MENDI

Ingeniero Aeronáutico 
Tribunal nombrado por el Sr. Rector Magfco. de la Universidad Politécnica de Madrid, el día... de. de $20 \ldots$

Presidente:

Vocal:

Vocal:

Vocal:

Secretario:

Suplente:

Suplente:

Realizado el acto de defensa y lectura de la Tesis el día .de. de $20 \ldots$ en la E.T.S.I. /Facultad.

Calificación

EL PRESIDENTE

LOS VOCALES

EL SECRETARIO 


\section{ACKNOWLEDGMENTS}

Quiero agradecer a mis compañeros de trabajo del Laboratorio de Técnicas Avanzadas de Ensayos del Área de Ensayos Aerodinámicos de INTA: Adelaida Garcia-Magariño, Mar Urdiales, Juan Carlos Martin, Juan Canales y Teodoro Gómez por la ayuda que me han prestado para la tesis así como por hacerme disfrutar de mi trabajo en mi carrera profesional en INTA. Al Dr. Mario Vargas y a Chris Lynch de NASA por su colaboración en los ensayos. A mi mujer Teresa Allegue por su apoyo y su ánimo. A mi madre Ángeles Mendi por todo su apoyo para terminar mis estudios. A mi director de tesis Ángel Velázquez por su trabajo, esfuerzo y saber sacar mayor provecho de los ensayos. A Jesús Morote jefe del Área de Ensayos Aerodinámicos por incentivarnos y facilitarnos la labor investigadora, y apoyarme para la realización de la tesis doctoral. A Alejandro Feo anterior jefe del Área de Ensayos Aerodinámicos por haberme facilitado su legado en el campo de la formación de hielo. Y por último agradecer especialmente a Adelaida GarcíaMagariño por su inestimable ayuda en la tesis y animarme a retomar la idea de hacer la tesis doctoral. 


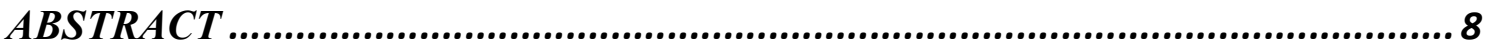

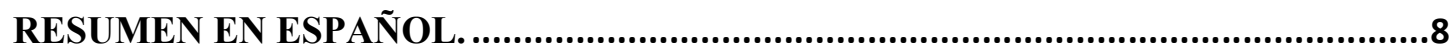

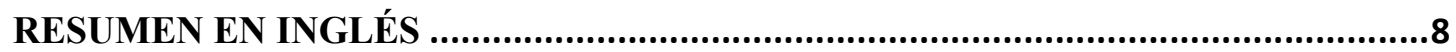

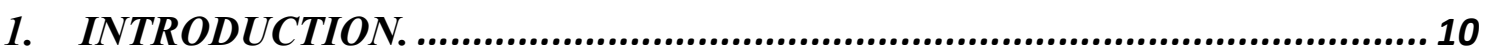

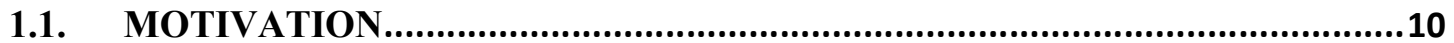

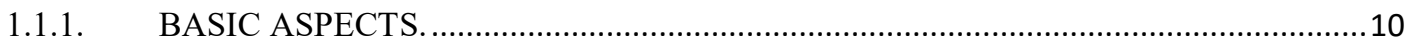

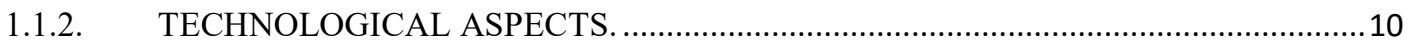

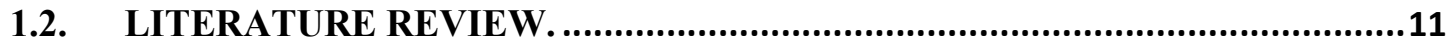

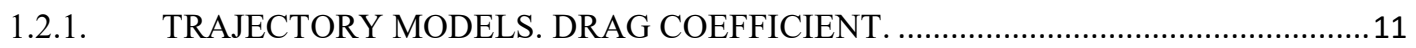

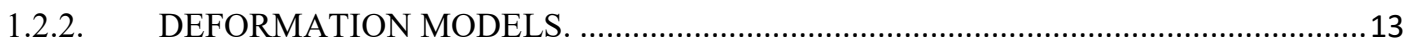

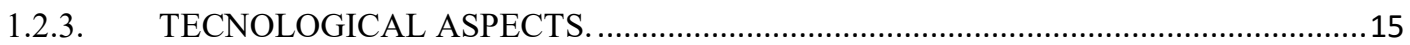

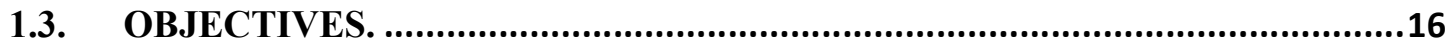

2. DESCRIPTION OF THE EXPERIMENTS......................................... 18

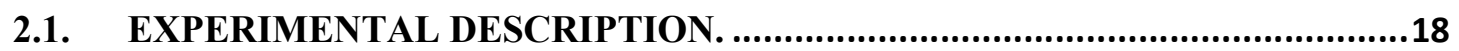

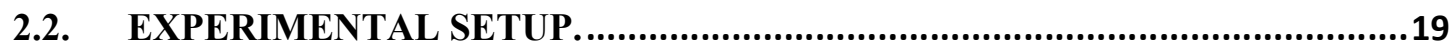

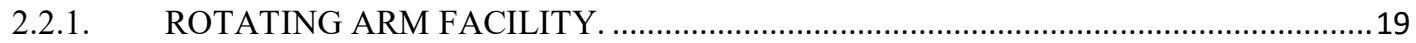

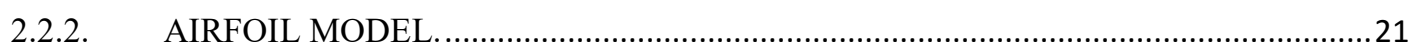

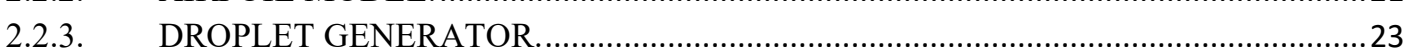

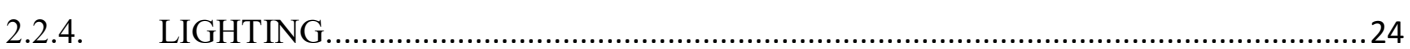

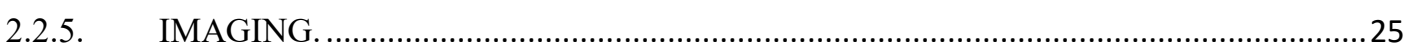

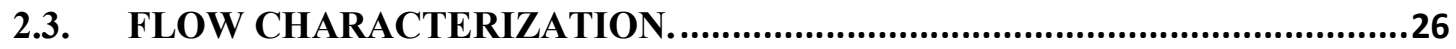

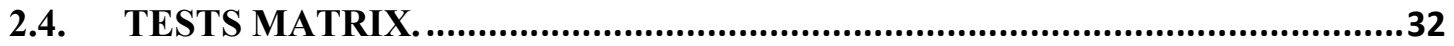

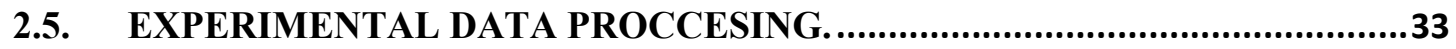

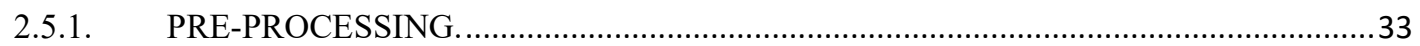

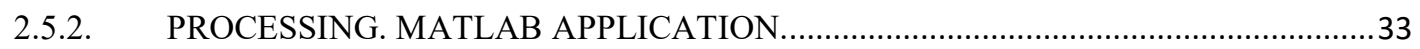

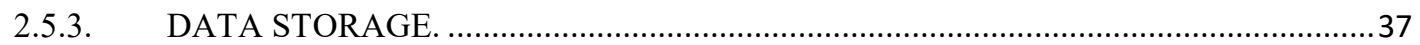

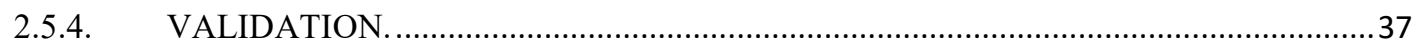

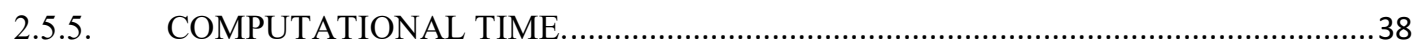

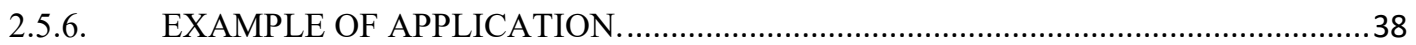

3. DROPLET TRAJECTORY AND DEFORMATION MODEL IN THE

STAGNATION LINE OF AN AIRFOIL................................................... 42

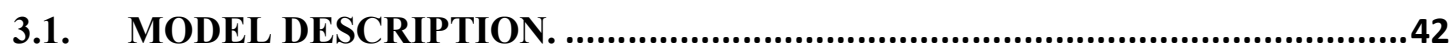

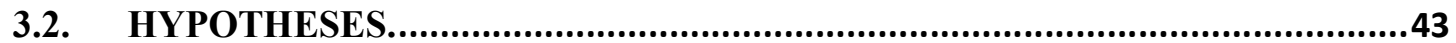

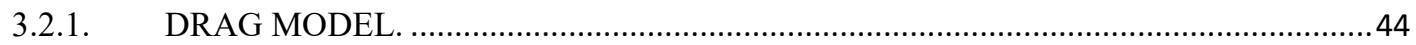

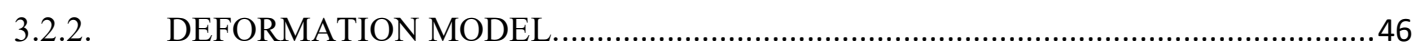

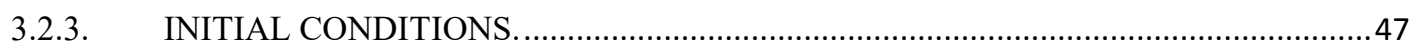

3.3. DIMENSIONLESS MODEL EQUATIONS. ......................................................

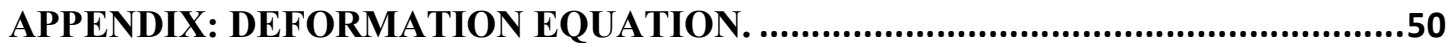

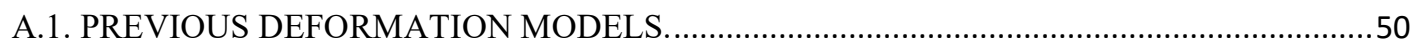

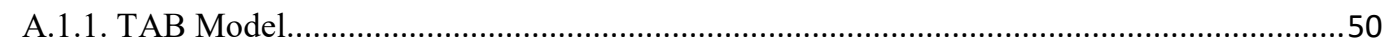

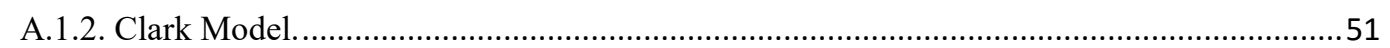

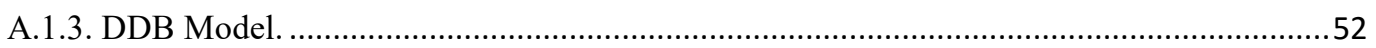




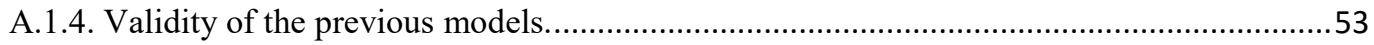

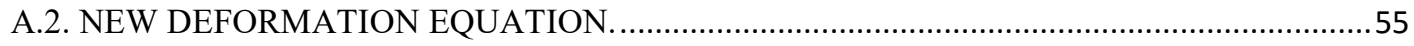

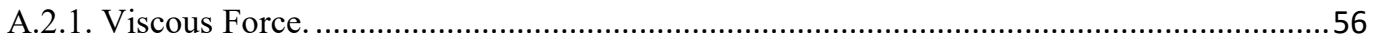

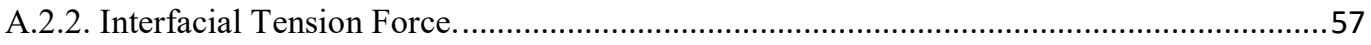

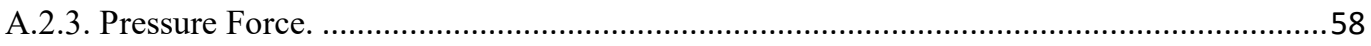

A.2.4. Equation for Droplet Ratio Deformation (DRD).....................................................59

A.2.5. Pressure Proportionality Coefficient Estimate............................................................ 60

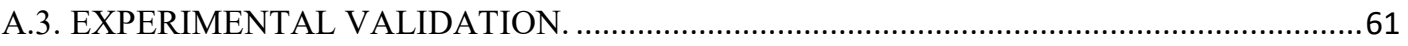

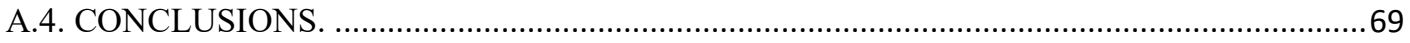

4. EXPERIMENTAL VALIDATION OF THE MODEL IN THE STAGNATION LINE IN THE VICINITY OF AN AIRFOIL................................................ 71

4.1. EXPERIMENTAL MATRIX................................................................

4.2. RESULTS AND DISCUSSIONS...............................................................

4.3. CONCLUSIONS...........................................................................................79

5. APLICABILITY OF THE MODEL TO PREDICT THE TRAJECTORY OF DROPLETS OUTSIDE THE STAGNATION LINE OF AN AIRFOIL................... 82

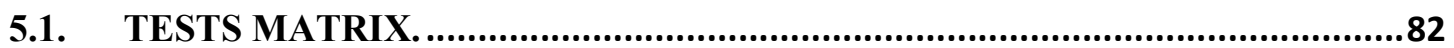

5.2. PARTICULARITIES OF THE STUDY REGION.............................................

5.3. EXPERIMENTAL RESULTS AND DISCUSSION.............................................

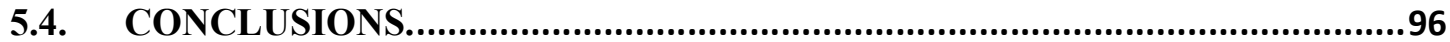

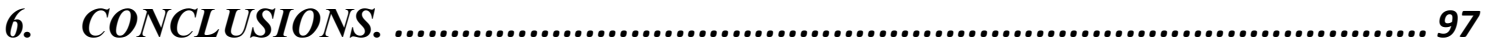

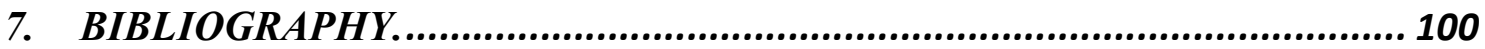




\section{ABSTRACT}

\section{RESUMEN EN ESPAÑOL.}

El principal objetivo de la tesis es proponer un modelo de trayectoria y deformación de gotas inmersas en un campo fluido continuamente acelerado. Asumiendo que la trayectoria de la gota viene definida por dos ecuaciones dinámicas que representan el equilibrio de fuerzas en dirección horizontal y en dirección vertical, y una tercera ecuación que modela la deformación de la gota, se ha considerado especialmente tres aspectos: el modelo del coeficiente de resistencia en un flujo no estacionario, el modelo de deformación de la gota inmersa en un campo fluido continuamente acelerado y el modelo de acoplamiento entre el coeficiente de resistencia y la deformación de la gota.

Para la validación experimental de este modelo se han realizado ensayos en la instalación del brazo rotatorio de INTA (Instituto Nacional de Técnica Aeroespacial Esteban Terradas). Los ensayos consistían en tomar imágenes de video de alta velocidad, mediante la técnica de imágenes de sombras, de gotas de agua en las inmediaciones del borde de ataque de un perfil en movimiento. Se han realizado ensayos con diámetros de gotas de agua comprendidas entre 300 micras y 1300 micras, cinco velocidades de modelo $(50,60$, 70,80 y $90 \mathrm{~m} / \mathrm{s}$ ) y tres tamaños de modelo. También se ha caracterizado los campos fluidos alrededor del borde de ataque de los tres modelos perfil mediante PIV (Particle Image Velocimetry). Debido a la gran cantidad de imágenes obtenidas en los ensayos, para el procesado de estas, se ha desarrollado un software en Matlab que permite obtener la trayectoria, la deformación de la gota y la evolución instantánea de distintos parámetros.

La validación del modelo de trayectoria se ha realizado en las inmediaciones de la línea de remanso del perfil en el que el flujo es continuamente acelerado, observándose una gran concordancia entre el modelo propuesto y los datos experimentales.

Por último se ha realizado un estudio sobre la posible aplicación de este modelo en el lomo del perfil, donde la velocidad del campo fluido varia no solo en modulo sino también en dirección, observándose mayores discrepancias debido principalmente a la interferencia entre gotas.

\section{RESUMEN EN INGLÉS.}

The main objective of the thesis is to propose a model of trajectory and deformation of droplets immersed in a fluid field continuously accelerated. Assuming that the trajectory of the drop is defined by two dynamic equations that represent the balance of forces in the horizontal direction and in the vertical direction, and a third equation that models the deformation of the drop, three aspects have been considered: the drag coefficient model in a unsteady flow, the drop deformation model immersed in a continuously accelerated 
fluid field and the coupling model between the drag coefficient and the deformation of the drop.

For the experimental validation of this model, tests have been carried out on the installation of the rotating arm of INTA (National Institute of Aerospace Engineering Esteban Terradas). The tests consisted of taking high-speed video images, using the shadow imaging technique, of water droplets in the vicinity of the leading edge of a moving airfoil. Tests with water droplet diameters ranging from 300 microns to 1300 microns, five model speeds $(50,60,70,80$ and $90 \mathrm{~m} / \mathrm{s})$ and three model sizes have been performed. Fluid fields around the leading edge of the three airfoil models have also been characterized by PIV (Particle Image Velocimetry). Due to the large number of images obtained in the tests, a Matlab software has been developed for the processing of these, allowing the trajectory, the deformation of the droplet and the instantaneous evolution of different parameters to be obtained.

The validation of the trajectory model was carried out in the vicinity of the stagnation line of the airfoil in which the flow is continuously accelerated, observing a great agreement between the proposed model and the experimental data.

Finally, a study has been carried out on the possible application of this model in the shoulder of the profile, where the velocity of the fluid field varies not only in modulus but also in direction, being observed greater discrepancies mainly due to the interference between drops. 


\section{INTRODUCTION.}

\subsection{MOTIVATION. \\ 1.1.1. BASIC ASPECTS.}

The aim of this thesis is to propose a trajectory and deformation model for droplets that are immersed in a flow field where the velocity and the acceleration that the droplet senses increase continuously. Three aspects need special consideration: the modeling of the droplet drag coefficient in an unsteady flow, the droplet deformation model and the coupling between the drag coefficient model and the deformation of the droplet.

The drag coefficient model for unsteady flow in previous studies only covered rigid spheres (or bodies) or small droplets where their deformation is neglected. In this thesis, water droplets distort becoming an oblate spheroid and their deformation is included to model the drag coefficient.

On the other hand, droplet deformation models that exist have been developed and validated for both falling droplets and droplets that are suddenly exposed to a constant air stream. These models do not reproduce accurately the deformation in continuously accelerating flows and therefore a new model of the deformation is presented in this thesis.

A complete trajectory and deformation model is proposed in this thesis. The novelty consists on considering the unsteady effects to model the drag coefficient, which in turn depends on the droplet deformation and a deformation model for droplets immersed in an accelerated flow field.

\subsubsection{TECHNOLOGICAL ASPECTS.}

The hazards of aircraft flying in heavy rain conditions or icing conditions are still under study. In particular in icing conditions, super-cooled large droplets, also known as SLD, are investigated in order to add a new appendix in the JAR/FAR regulations. These larger droplets experienced phenomena such as deformation prior to impact that need to be considered.

An important issue to be solved is to model the trajectory of the droplets in order to know the impact position and the distribution of the water droplet reaching the leading edge of the airfoil. In these conditions, droplets in the cloud are exposed to an accelerating flow field that continuously increases as the wing approach them. 
Therefore, the flow field studied in this thesis is a continuously accelerated flow field. To this end, an experimental study is conducted in a rotating arm, where droplets are intercepted by an incoming airfoil.

\subsection{LITERATURE REVIEW. \\ 1.2.1. TRAJECTORY MODELS. DRAG COEFFICIENT.}

The deformation of the droplets leads to a change in their drag coefficient (Jenkins and Booker 1965, Crowe et al 1963, Ortiz et al 2004, Simpkins and Bales 1972, Luxford et al 2005), which in turn modifies its resistance and therefore the final trajectory of the drop. There are several expressions of the drag coefficient for droplets deformed by airflows in the literature. Jenkins and Booker (1965) consider that a droplet of initial radius $R_{0}$ that is accelerated and disintegrated by an air stream $U$ has the same acceleration as a drop of constant radius $R_{0}$ subjected to a constant relative velocity $\mathrm{U}$ with an equivalent constant drag coefficient of 2.26. Ortiz et al. (2004) provide a correlation for droplets subjected to a high velocity stream between the value of the drag coefficient $C_{D}$ and the values of Ohnesorge number $O h$ and the Weber number $W e$,

$$
C_{D}=1.6+0.4 \cdot O h^{0.08} \cdot W e^{0.01}
$$

The Ohnesorge number $O h$ and the Weber number $W e$ are defined as usual

$$
\begin{gathered}
O h=\frac{\mu_{l}}{\sqrt{\rho_{l} \sigma d}} \\
W e=\frac{\rho_{a} U^{2} d}{2 \sigma}
\end{gathered}
$$

where $\mathrm{d}$ is the initial droplet diameter, $\sigma$ is the surface tension, $\mu_{l}$ is the viscosity of the liquid droplet and $\rho_{a}$ is the air density.

Simpkins and Bales (1972), after obtaining shock tube experimental data and comparing it with similar previous data, provide a mean drag coefficient of 2.5 for Reynolds between $10^{3}$ and $10^{5}$. Liu et al. (1993) give a value for the drag coefficient of a deformed drop as a function of the drag coefficient of a sphere and the deformation of the drop

$$
C_{D_{\text {droplet }}}=C_{D_{\text {sph }}}(1+2.632 \cdot y)
$$

where $y$ is the displacement of the equator of the droplet. Luxford et al. (2005) model the drag coefficient of a deformed drop by assuming an interpolation between the drag 
coefficient of a sphere and that of a flat disk. When the droplets, instead of being subjected to a uniform velocity stream, are subjected to an exponentially increasing velocity flow over time, as in the case of droplets near an incoming profile, their drag coefficient varies due to the acceleration of the flow field (Vargas et al 2013). Some experimental studies and discussions on drag coefficient variation due to the non-stationarity of the flow field can be found in the works of Odar and Hamilton (1964), and Karanfilian and Kotas (1978).

Regarding, now, more basic fluid mechanics aspects of the modeling of droplet trajectories, it is worth mentioning, first, the review article published back by Aggarwal and Peng (1995). In section 1 of that article, the authors review a number of droplet dynamic models and conclude that flow non-uniformity and acceleration certainly affect forces on droplets. However, they report a large uncertainty regarding their actual quantitative contribution to total drag and lift forces. Even though it is not a review article by itself, Schmehl (2002) also contains a quite interesting discussion on droplet drag and dynamics models, including those models that deal with flow non-uniformity and acceleration. A method to reduce to a minimum the number of similarity parameters needed to close a droplet trajectory model (within the realm of certain assumptions) has been published by Bragg (1982). In the case of unsteady Stokes flow, Maxey and Riley (1983) have proposed a generalized equation of motion for a sphere in a non-uniform flow. In the case of an inviscid unsteady non-uniform flow, Auton et al (1988) have derived a general expression for the fluid force on the body. The case of droplet deformation under steady conditions for a variety of fluids other than water has been extensively studied experimentally by Hsiang and Faeth (1995). Empirical correlations for the drag coefficient of gas bubbles inside a liquid (the opposite case to the one presented here) have been reported by Zhang et al. (2008). A statistical approach to droplet trajectory prediction during aerodynamics fragmentation has been developed by Flock et al (2012). Because of its interest from the standpoint of novel experimental techniques, it is also worth mentioning the work of Zarrabeitia et al (2015). Zarrabeitia et al developed a new method for extracting 3D flight trajectories of droplets using highspeed stereo capture. They proposed four droplet flight models to reconstruct the trajectory of the droplet from the initial information. The drag is only taken into account in two of them being one linearly proportional to speed and the other one quadratic to speed. Droplet deformation and unsteady effects are not considered due to the small velocities.

Because of their relevance for the present work, it is important to discuss in some detail the studies presented by Temkin and Metha (1982), Igra and Takayama (1993), and Jourdan et al (2007). The article by Temkin and Metha (1982) presents an experimental study on the motion of water droplets inside accelerating and decelerating flows. The interesting modeling aspect of this study is that the authors assume a functional relation between the drag coefficient and the so called acceleration parameter that is defined as the time derivative of the slip velocity divided by the square of the slip velocity itself. 
The experiments were carried out in a shock tube. Droplet diameters were in the range from $100 \mu \mathrm{m}$ to $200 \mu \mathrm{m}$, which led to Weber numbers such that the authors assumed a negligible droplet deformation. Their conclusion (which is in contradiction to some other studies published in the literature) is that the unsteady drag of a sphere in decelerating flow is always larger than the steady drag at the same Reynolds number, while is it always smaller if the surrounding flow accelerates. Igra and Takayama (1993) also used a shock tube facility but, in their case, with non-deformable spheres made up of Polystyrene, Nylon, and Polyamide-imide. Their diameter ranged from $0.5 \mathrm{~mm}$ to $4.8 \mathrm{~mm}$. The Reynolds number covered in their experiments was in the range down from 6,000 up to 100,000. Incident Mach numbers in the shock tube were 1.27, 1.50, and 1.80. In their conclusions, the authors reported unsteady drag values about $50 \%$ larger than the corresponding steady values in these shock tube conditions. More recently, Jourdan et al (2007) have presented another quite comprehensive experimental study based, also, on a shock tube type test rig. They also used non deformable spheres (made of either polystyrene or nylon) with diameters ranging from $500 \mu \mathrm{m}$ to $6.6 \mathrm{~mm}$. In their tests, the authors found that the unsteady drag is always larger than the steady drag at the same Reynolds number and explicitly stated at the end of their "Results and Discussion" section (section 4) that the acceleration parameter proposed by Temkin and Metha (1982) may not be the relevant characteristic parameter for the flows that they considered. Notwithstanding the fact that both experimental studies presented some differences that might affect the conclusions (droplet were deformable in Temkin and Metha (1982) and non-deformable in Igra and Takayama (1993), and velocities, and associated compressibility effects, were also somewhat different) this shows that the field is alive and that no definite conclusions are available so far.

\subsubsection{DEFORMATION MODELS.}

Droplet deformation is a subject of interest both from the basic Fluid Mechanics and from the engineering points of view. Regarding fundamental Fluid Mechanics, one of the main challenges of droplet deformation and breakup research is to generalize the different modes of deformation. At the early stages of this type of research, the deformation modes were characterized based on qualitative features. More recently, different attempts have been tried to indentify a limited set of fundamental mechanisms that manifest themselves in the fashion of a wide variety of different deformation modes. For a comprehensive review of this type of approach, the interested reader is directed to the work of Theofanous (2011) and Theofanous and Li (2008). Another basic Fluid Mechanics aspect that has attracted the attention of researchers has been the influence that the flow velocity profile has on the droplet deformation and breakup processes. Although many theoretical and experimental studies (based, mostly, on shock tube type facilities) have been carried out in conditions of constant, or nearly constant, flow velocity, certain applications of engineering interest are characterized by either accelerating or decelerating flow profiles. In this specific area, relevant references are the works of Temkin and Metha (1982), Igra and Takayama (1993) and Jourdan et al. (2007). 
There are authors such as O'Rourke and Amsden (1987), Clark (1988), Ibrahim et al. (1993) that propose models of semiempirical deformation from establishing an analogy between a drop and a mass-spring system. This analogy was first proposed by Taylor (1963), consisted of considering two equivalent masses located in the mass centers of each half of the drop, joined by a spring and a damper. The stiffness of the spring represents the surface tension of the drop, the damper represents the viscosity and the aerodynamic forces are those that act on the masses. The main models of deformation of droplets that depart from this analogy are:

1) Taylor Analogy Breakup Model (TAB): This model, whose present dimensionless form was proposed by O'Rourke and Amsden (1987), is widely accepted in the scientific community (Liu et al 1993). Using experimental data and theoretical considerations, the four terms that make up the equation of the mass-spring system are obtained.

2) Clark's Model (Clark (1988)): The Clark model is a correction of the TAB model, where two masses are considered concentrated in each halves of the drop. The forces acting on the drop are obtained by a linear two-dimensional approximation assuming the cross section of the drop constant. By means of mainly theoretical considerations, each of the forces are postulated and linearized, finally arriving at a second-order linear equation analogous to that of a mass-spring system.

3) Droplet Deformation and Breakup Model (DDB): This model (Ibrahim et al 1993) is a three-dimensional approach that assumes that the drop is distorted due to a pure extensional flow field from an initially spherical shape to an oblate spheroid. The dynamics of the droplet are formulated in terms of the half-droplet mass center equation. The problem is addressed by the energy equation, neglecting the heat exchange of the droplets with their surroundings. The internal energy comes from the kinetic energy and the potential energy is due to the surface tension of the drop. The work is performed by the pressure forces and dissipation is due to viscosity. Many authors refer to this model and made use of it (Hwang et al 1996, Liu and Reitz 1997, Park et al 2002, Pham and Heister 2002, Park and Lee 2004, Lee et al 2004).

These three models are normally used in droplets subjected to uniform stream of air generated in shock tubes or wind tunnels. Ibrahim compares all three models with Krzeczkowski's experimental data (Krzeczkowski 1980) and seems to get improvements with his DDB model. However, there were some errors in his results, as corrected by Lee et al. (2012). These models had been applied to droplets in uniform air stream, until Vargas (2012) tried to apply the DDB model to experimental data of deformed droplets 
in a flow field accelerated. To this end, he assumed that at each instant the response time of the drop was smaller as compared to the change in the flow velocity, thus remaining with the stationary terms. It obtained as a result that for large droplets (diameters greater than $1 \mathrm{~mm}$ ) there were notable discrepancies between the predicted results and the experimental values. It is obvious, then, to develop new models that account for the truly unsteady nature of the problem, as it is the case of droplets in the vicinity of a leading edge of an incoming airfoil. Another more recent model was proposed by Marek (2013). However, this model, as explicitly argued by the author only covered the case of impact interaction when the drop is suddenly exposed to the flow motion, as in shock tube experiments.

\subsubsection{TECNOLOGICAL ASPECTS.}

The problem of predicting liquid droplet trajectories, inside a gas flow is of interests in many fields of science and engineering. They include, among many others, forensic applications (Kabaliuk et al 2014), solvent extraction (Petera et al 2007), electrostatic enhancement of liquid-liquid contacting processes (Petera et al 1997), ink-jet printer design (Mohebil and Evans 2005), spray modeling (Sinha et al 2015, Taskiran and Ergeneman 2014), and design of nuclear fusion subsystems (Yang et al 2013).

In aeronautics, computation of water droplet trajectories is of interests, among others, for the purpose of simulating icing conditions. As compared to situations in other technical fields, droplet trajectories in these aeronautics-type conditions are characterized by the fact that, in the vicinity of an incoming airfoil, the flow, as seen from the reference frame of the droplet, accelerates with a non-constant acceleration. This is in contrast to other cases in which the flow is steady or accelerates with constant acceleration. In this context of dealing with non-uniform flows from a numerical point of view, the interested reader is directed to the work of Rendall and Allen (2014) that developed a finite volume code in which droplet motion is tracked using mesh connectivity. This work is of interest because, instead of using a pure Lagrangian approximation, the authors couple the droplet motion to the finite volume computation of the surrounding flow in a computationally efficient way. Also of interest is the work by Saeed et al (2005) in which the air part of the code is of the panel method type, thereby allowing for a much faster computation of droplet trajectories, albeit at the expense of a smaller accuracy. On the other hand, a comprehensive work that involves both theoretical modeling and experimental testing has been reported by Papadakis et al. (2007) while yet more experimental can be accessed in the study published by Reehorst and Ibrahim (1995).

With regard to aeronautics applications, the issue of droplet deformation and breakup has to do with two different aspects related to flight safety. The first one is the effect of heavy rain on flight controllability, while the second one is its influence on icing conditions. Statistics show, Cao et al (2014), that about $50 \%$ of the accidents in civil aviation are 
connected to adverse atmospheric conditions. A large percentage of these (not easily quantified) involved heavy rain, Krezel et al (2008). The reason is that heavy rain impact on the forward part of the fuselage and on the wings affects the torque around the aircraft center of gravity, Cao et al (2014). Also, droplet impact and accumulation on wings and control surfaces generates an "artificial roughness" effect that degrades controllability. For example, Hansman and Craig (1987) have shown that rain with a water content of 30 $\mathrm{gr} / \mathrm{m}^{3}$ degrades the aerodynamic behavior of NACA 64-210 and NACA 0012 airfoils by a factor of $5 \%$ and $15 \%$ respectively. Heavy rain may also clog the gap in a lifting surface with deflected slats, thereby causing premature boundary layer separation, Zhang and Cao (2010). Also, Reehorts et al (2000), have estimated that icing caused 542 flight accidents between 1976 and 1988. This is because ice accretion deteriorates aerodynamic performance by a factor of the order of 5\% to 25\%, Lampton and Valasek (2012). Additionally, icing may contribute to the anticipation of dynamic stall, Thomas et al (2003).

For all these reasons it is important to develop engineering tools that help aircraft manufactures to quantify the influence of these effects on the systems and products that they design and manufacture. In this context, no matter whether the tools are based on semi empirical correlations or on computational fluid dynamics approaches, it is important to have reliable quantitative data (either for correlation or for validation purposes) on droplet deformation and breakup in the vicinity of lifting and/or control surfaces. However, for aircraft related applications, data obtained in shock tubes with constant incoming flow velocity does not faithfully represent the actual situation in the vicinity of an approaching airfoil. The reason is that in the droplet frame of reference, the incoming flow field continuously accelerates, even if the airfoil moves at constant velocity. This is the reason why, when planning for aeronautics applications, dedicated facilities, such as rotating arms, need to be used.

\subsection{OBJECTIVES.}

The general objectives of the thesis are:

1. To develop a theoretical model to predict the trajectory and deformation of droplets subjected to a flow field with an increasing acceleration.

2. To validate the model with experimental data of water droplets in the stagnation line of a moving airfoil.

3. To study the applicability of the model in the shoulder region of a moving airfoil.

The specific objectives are: 
1. To provide experimental data on droplet trajectory and deformation of water droplets of diameters from 400 to $1500 \mu \mathrm{m}$ in the stagnation line of an airfoil. Three different airfoil models and five model velocities were used.

2. To develop a complete trajectory equation model that includes the modeling of the drag coefficient model and the deformation for water droplets in the stagnation line of an incoming airfoil.

3. To validate the model for water droplets in the stagnation line of an incoming airfoil.

4. To provide experimental observation visualization on droplet trajectory and deformation of water droplets of diameters of $1000 \mu \mathrm{m}$ in the shoulder region of the leading edge of an airfoil. One airfoil model and three model velocities were used.

5. To apply the model in the shoulder region of an incoming airfoil and study its validity. 


\section{DESCRIPTION OF THE EXPERIMENTS.}

\subsection{EXPERIMENTAL DESCRIPTION.}

In order to assess the droplet trajectory and deformation model in a continuously accelerated flow field, water droplets near the leading edge of an incoming airfoil are used. To this end, a stream of droplet generated in a controller manner by a monodisperse droplet generator is allowed to fall in the path of an incoming airfoil mounted at the end of a rotating arm. High-speed video shadowgraph image technique used for data acquisition and post-processing by an in-house software allow obtaining quantitative data.

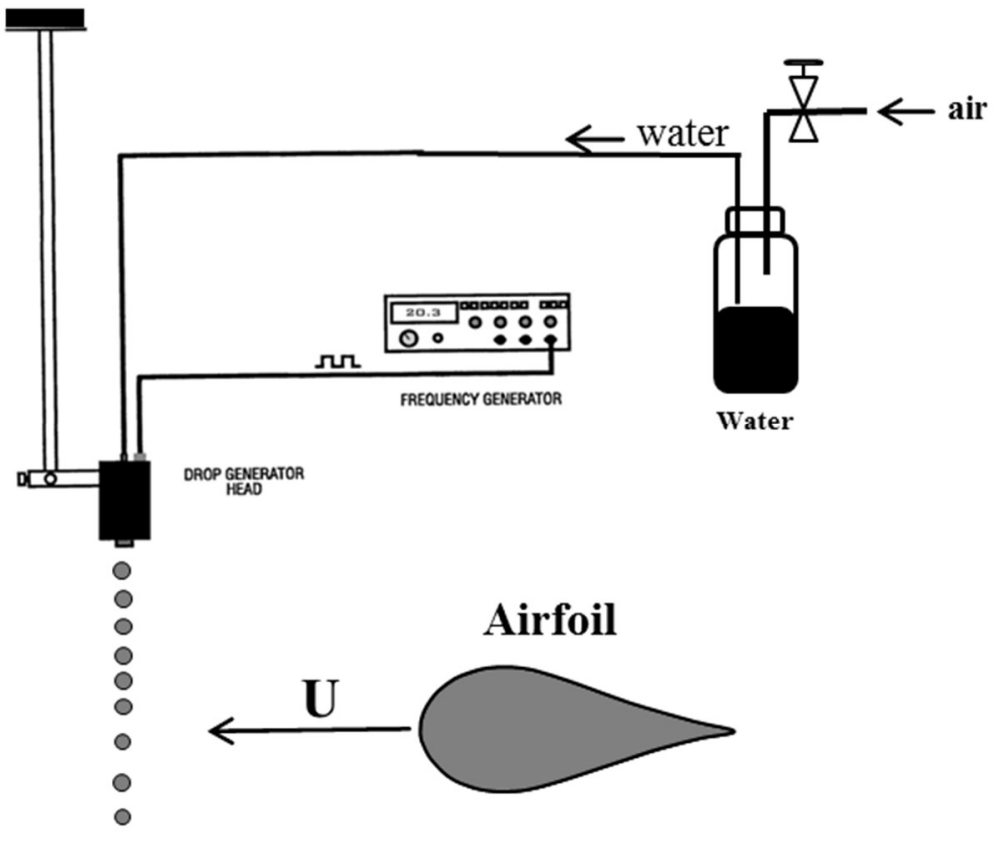

Figure 2.1.1 Conceptual approach of the experiments 


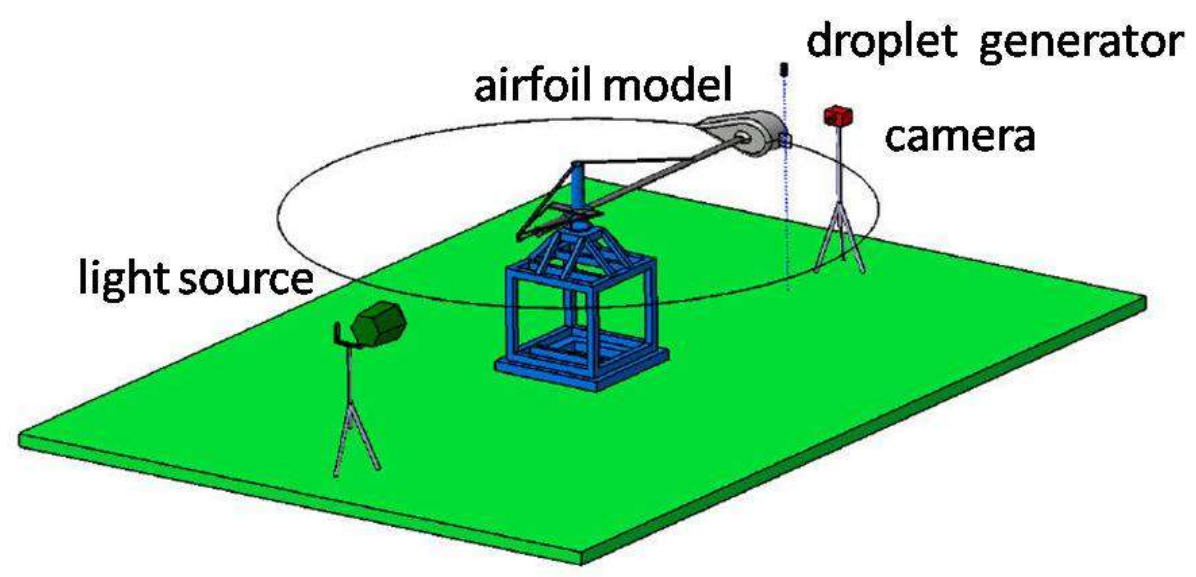

Figure 2.1.2. Sketch of the facility.

\subsection{EXPERIMENTAL SETUP. 2.2.1. ROTATING ARM FACILITY.}

The experimental campaigns to test the droplet trajectory model have been carried out at the INTA rotating arm facility in Spain.

The facility, see figures 2.2.1.1 and 2.2.1.2, consists of an electric motor, a support structure and a rotating arm that has a length of $2.2 \mathrm{~m}$. The engine axis is vertical which means that airfoils placed at the arm's end describe a circular trajectory with diameter of $4.4 \mathrm{~m}$ in a horizontal plane. The whole setup is placed inside a room whose dimensions (length, width and height) are $6.8 \mathrm{~m}, 6.6 \mathrm{~m}$, and $2.7 \mathrm{~m}$, respectively. At maximum power the electrical motor can rotate at $400 \mathrm{rpm}$ which means the maximum attainable airfoil velocity is $90 \mathrm{~m} / \mathrm{s}$ (Mach 0.26$)$. 


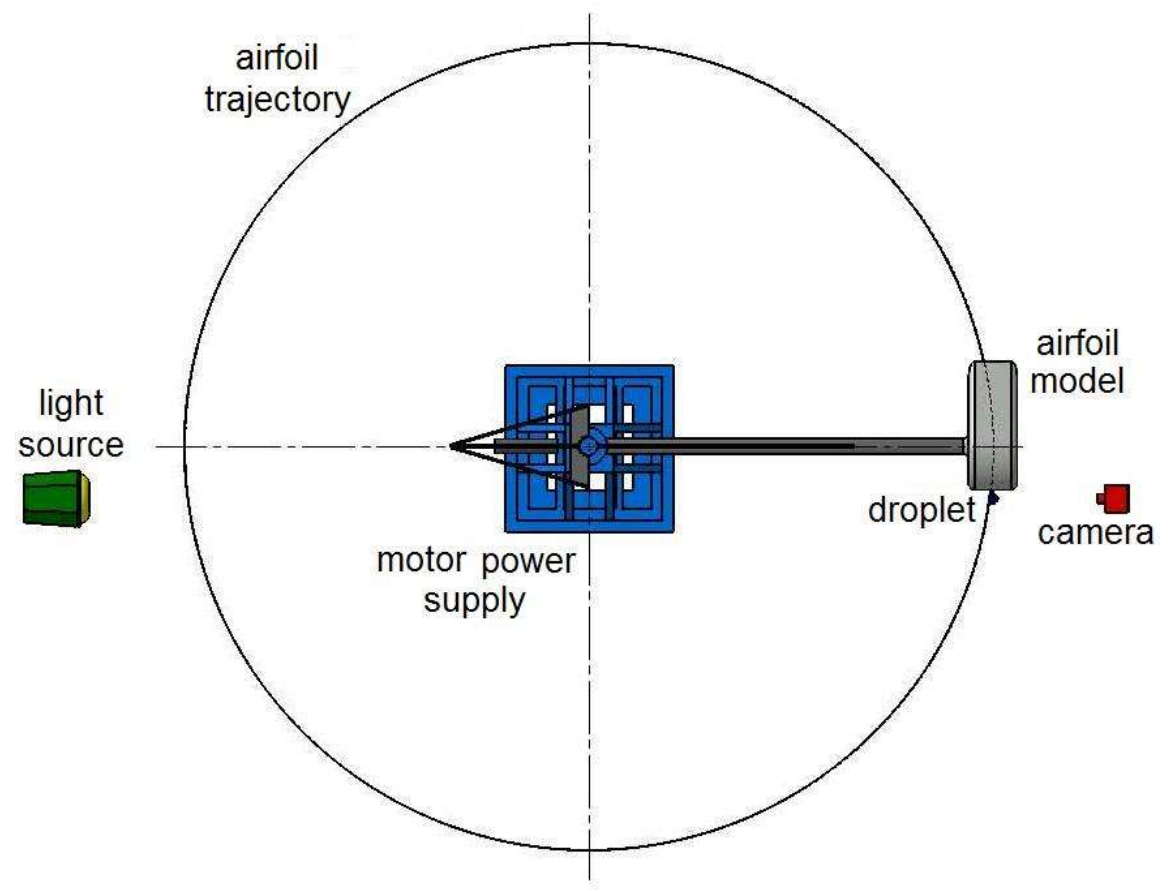

Figure 2.2.1.1. Top view (sketch) of the rotating arm facility.

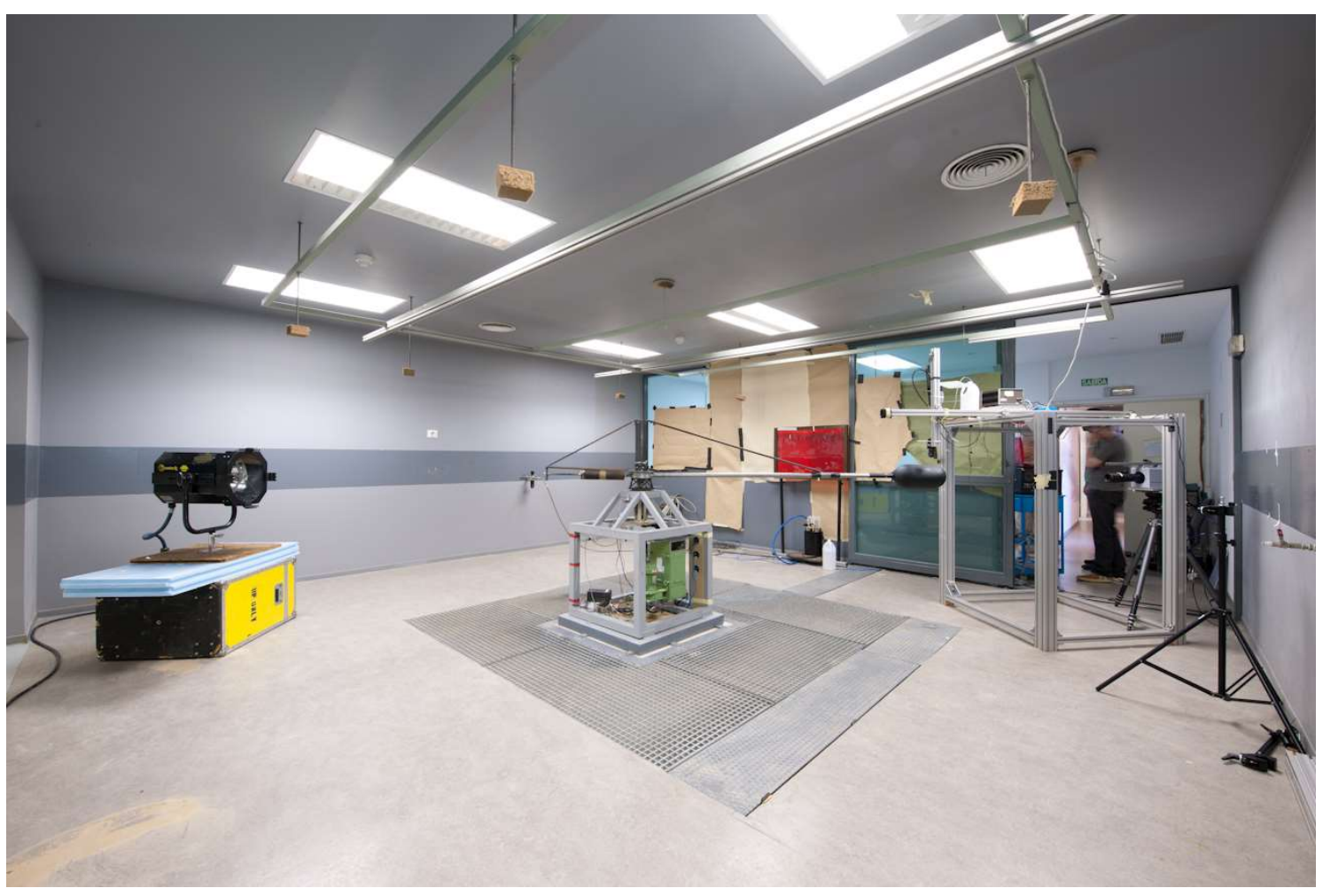

Figure 2.2.1.2. Actual picture of the rotating arm facility 


\subsubsection{AIRFOIL MODEL.}

The model is one of the most important components of the rotating rig facility. Mechanical and aerodynamic elements enter in its design. Due to the large centrifugal forces present, the mass needs to be carefully adjusted to the possible minimum, selecting the appropriate low density material supplemented with light metallic materials of enough structural strength in the model interior to maintain its integrity and sustain the union to the arm. Another important aspect in relation with the intended simulation in the experiments is the aerodynamic design. The air flow forces need to be maintained low so that the required velocity can be attained, considering the power constraints imposed by the electric motor and keeping the associated aerodynamic wake effects to the minimum.

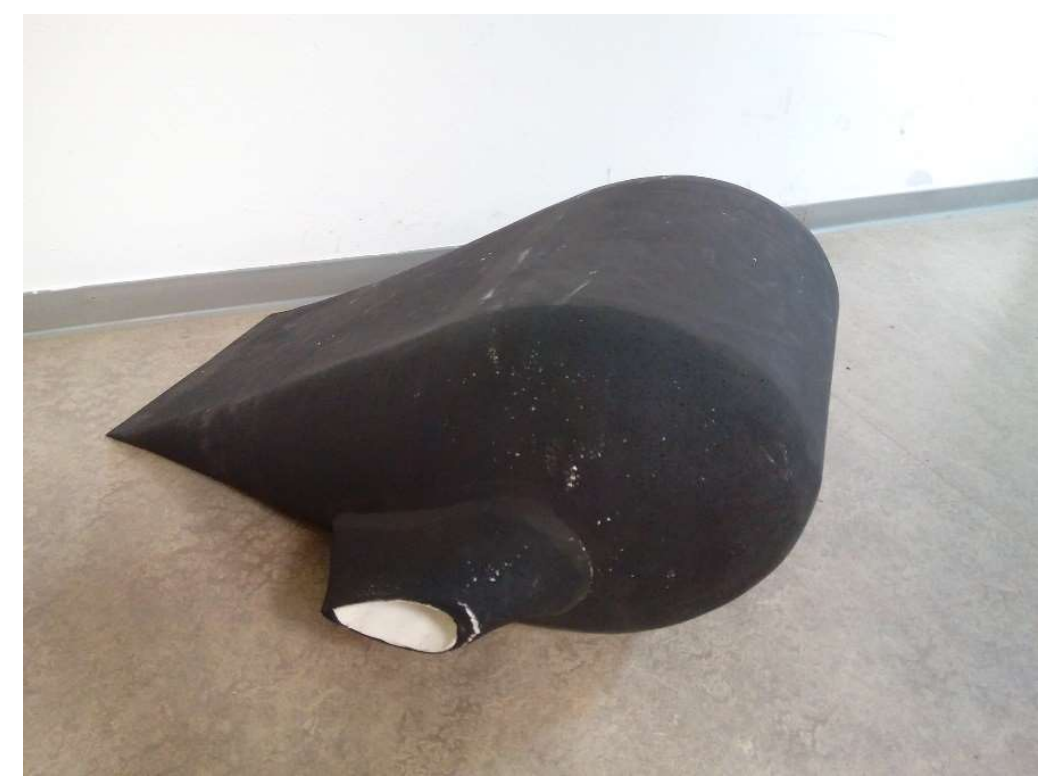

Figure 2.2.2.1. Airfoil Model.

The airfoils were rather blunt to simulate the ones used for commercial aircraft design (see figure 2.2.2.1). The dimensionless coordinates of the profile are given in table 2.2.2.1, while its actual shape is presented in figure 2.2.2.2. 


\begin{tabular}{cccc}
\hline $\mathbf{x} / \mathbf{c}$ & $\mathbf{z} / \mathbf{c}$ & $\mathbf{x} / \mathbf{c}$ & $\mathbf{z} / \mathbf{c}$ \\
\hline 1.000 & 0.000 & 0.241 & 0.200 \\
0.937 & 0.014 & 0.211 & 0.196 \\
0.877 & 0.028 & 0.183 & 0.191 \\
0.818 & 0.044 & 0.157 & 0.183 \\
0.762 & 0.059 & 0.133 & 0.173 \\
0.707 & 0.076 & 0.110 & 0.162 \\
0.655 & 0.093 & 0.090 & 0.150 \\
0.604 & 0.110 & 0.072 & 0.136 \\
0.556 & 0.127 & 0.056 & 0.121 \\
0.510 & 0.143 & 0.042 & 0.105 \\
0.465 & 0.158 & 0.030 & 0.089 \\
0.423 & 0.171 & 0.020 & 0.072 \\
0.383 & 0.183 & 0.012 & 0.055 \\
0.344 & 0.192 & 0.006 & 0.041 \\
0.308 & 0.198 & 0.002 & 0.032 \\
0.274 & 0.200 & 0.000 & 0.000 \\
\hline
\end{tabular}

Table 2.2.2.1. Dimensionless profile shape coordinates. "c" stands for the chord length. The leading edge coordinates are: $\mathrm{x} / \mathrm{c}=0, \mathrm{z} / \mathrm{c}=0$.

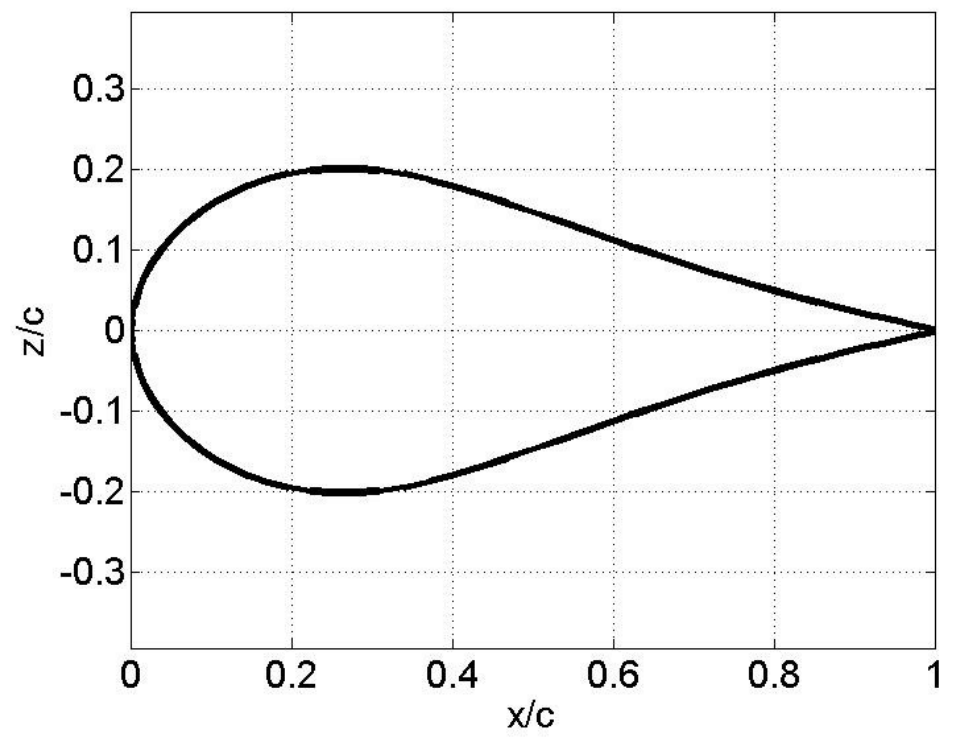

Figure 2.2.2.2. Airfoil profile shape

Three Styrofoam airfoils were used in the experiments. They were labeled M1, M2 and M3 and their geometry parameters are specified in table 2.2.2.2. 


\begin{tabular}{cccc}
\hline Model & Chord & $\begin{array}{c}\text { Leading Edge } \\
\text { Radius }\end{array}$ & Thickness \\
\hline M1 & $0.690 \mathrm{~m}$ & $0.103 \mathrm{~m}$ & $0.276 \mathrm{~m}$ \\
M2 & $0.468 \mathrm{~m}$ & $0.070 \mathrm{~m}$ & $0.187 \mathrm{~m}$ \\
M3 & $0.199 \mathrm{~m}$ & $0.030 \mathrm{~m}$ & $0.080 \mathrm{~m}$ \\
\hline
\end{tabular}

Table 2.2.2.2. Geometry parameters of the three airfoils: M1, M2, and M3

\subsubsection{DROPLET GENERATOR.}

Mono-disperse droplet generators operate through vibration of a water jet coming out of a circular orifice (see figure 2.2.3.1). If a laminar liquid jet with a flow rate $Q$ is vibrated at frequency $f$, it produces at short distances from the orifice an equally spaced stream of droplets that follow the expression (TSI 2012):

$$
d(\mu m)=317\left(\frac{Q\left(\frac{c c}{\min }\right)}{f(k H z)}\right)^{1 / 3}
$$

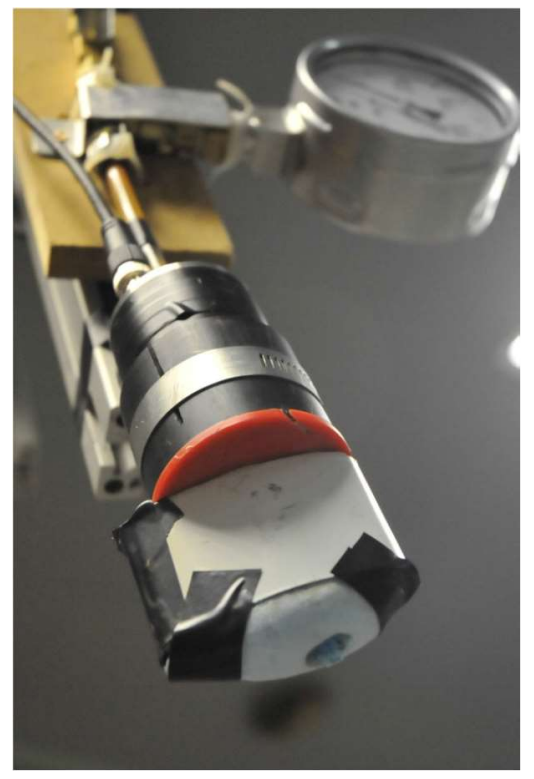

Figure 2.2.3.1. Droplet generator with aerodynamic protection.

The range of droplet sizes for a fixed orifice diameter is limited by the liquid jet velocity that stays in the laminar regime, indicating the limit of operation for that orifice. To increase the range of droplet diameters, larger orifice diameters need to be used. There is a group of frequencies (Raleigh frequencies) that follow the expression (TSI 2012): 


$$
f_{R}=\frac{U_{j}}{4.508 \cdot D_{j}}
$$

where $U_{j}$ is the water jet exit velocity and $D_{j}$ is the jet diameter (assumed to be equal to the orifice diameter), that produce a longer stable stream of droplets with diameter approximately twice the jet diameter. The basic system consists of a stainless steel water pressure container, connected through plastic tubing to a precision control valve with a manometer, to supply constant water pressure to the vibrating head directed to the exit orifice. The frequency generator prepared for this application was a $5 \mathrm{MHz} \mathrm{B} \& \mathrm{~K}$ Precision model 4011A. A sketch of the system is shown in figure 2.2.3.2. While in operation with the arm and model in rotation, an aerodynamic protector can be attached to the vibration head orifice exit to minimize external air disturbances that may promote premature breakup of the water jet into undesirable droplet sizes before it gets into the flow field induced by the model (see figure 2.2.3.1).

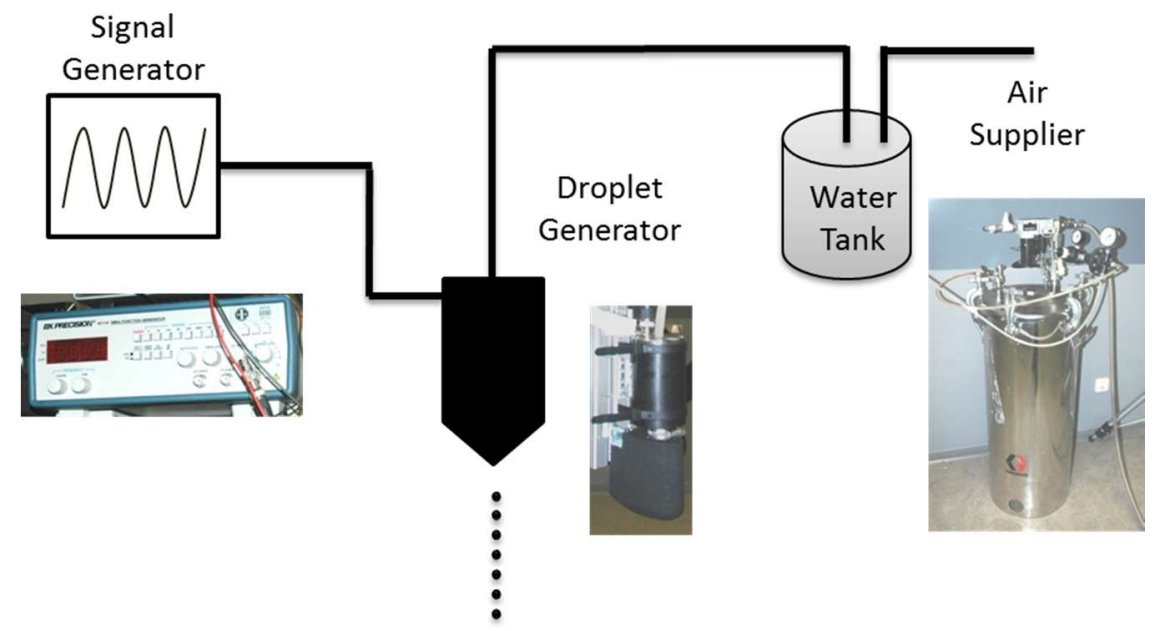

Figure 2.2.3.2. Droplet generator.

The droplet generator was a monosized TSI MDG-100. The discharge could be varied between $2.2 \mathrm{~cm}^{2} / \mathrm{min}$ and $51.79 \mathrm{~cm}^{2} / \mathrm{min}$. For the present experimental campaigns, three different ranges of droplets diameter were selected. They were $575 \mu \mathrm{m} \pm 25 \mu \mathrm{m}, 775 \mu \mathrm{m}$ $\pm 25 \mu \mathrm{m}$ and $1025 \mu \mathrm{m} \pm 25 \mu \mathrm{m}$. These specific ranges were obtained by means of acting on the jet mass flow rate of the droplet generator and the frequency of the piezoelectric.

\subsubsection{LIGHTING.}

Lighting equipment is another of the essential elements of the facility. The lighting instrumentation requirements are imposed by higher velocities and smaller droplets. Depending on the test objectives, high-intensity-continuous or short duration lights may 
be used. For droplet breakup and impact research experiments, the minimum time required is in the order of microseconds and the equipment used should be prepared to supply high intensity light during that order of magnitude or less. If the tests require highspeed video equipment, as it is the case, frame rates from 50,000 to 150,000 per second would be needed with high-intensity lighting to capture images in sufficient resolution. Simple direct backlight was used to satisfy the requirement. A xenon $2000 \mathrm{~W}$ lamp was used.

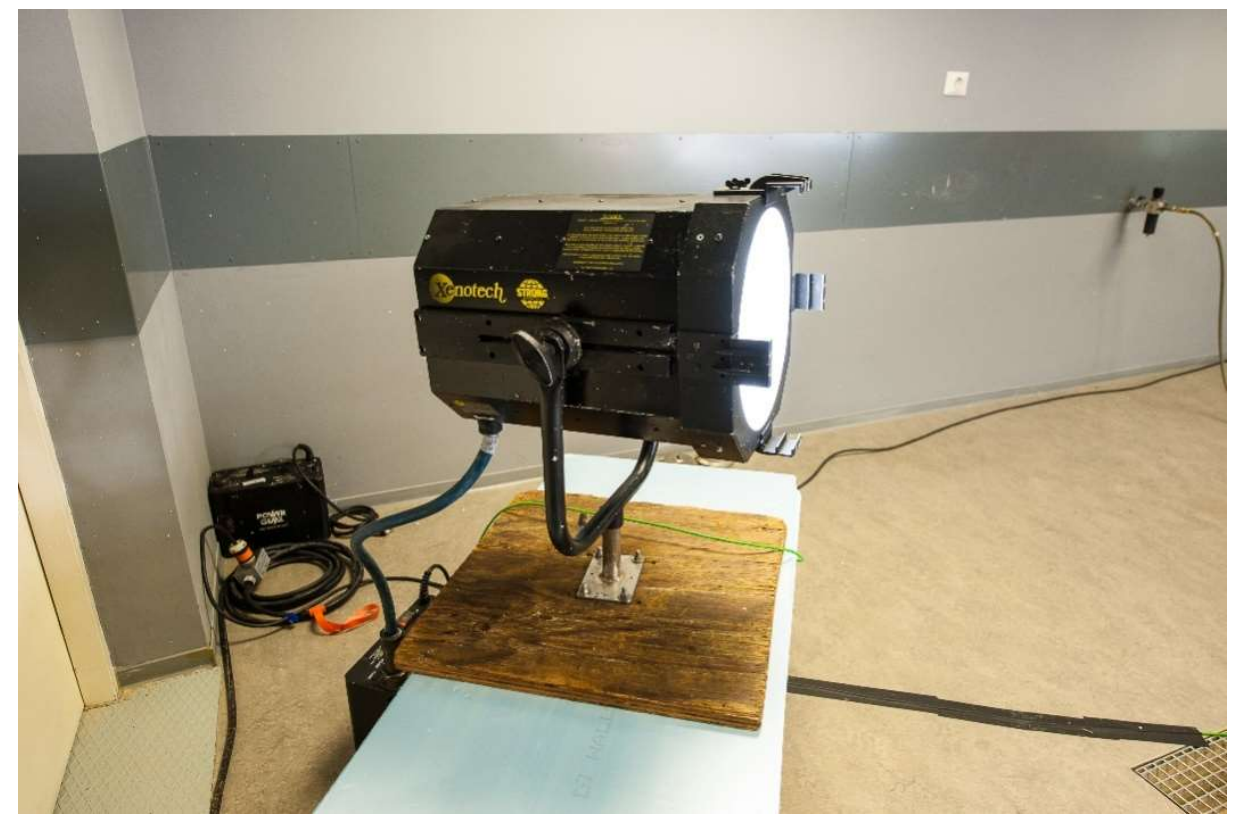

Figure 2.2.4.1. Xenon Lamp.

\subsubsection{IMAGING.}

Visualization is the basic technique in the facility, from which measurements have to be obtained and interpreted to analyze the phenomena under consideration and characterize it quantitatively. Due to the small droplet sizes involved, care must be exercised in choosing the appropriate high quality lens, extension rings and magnification to achieve maximum resolution compatible with reasonable field of view, to permit measurement and analysis of the pertinent characteristics: frame rate selection, total number of frames and synchronization of the starting frame with model location. 


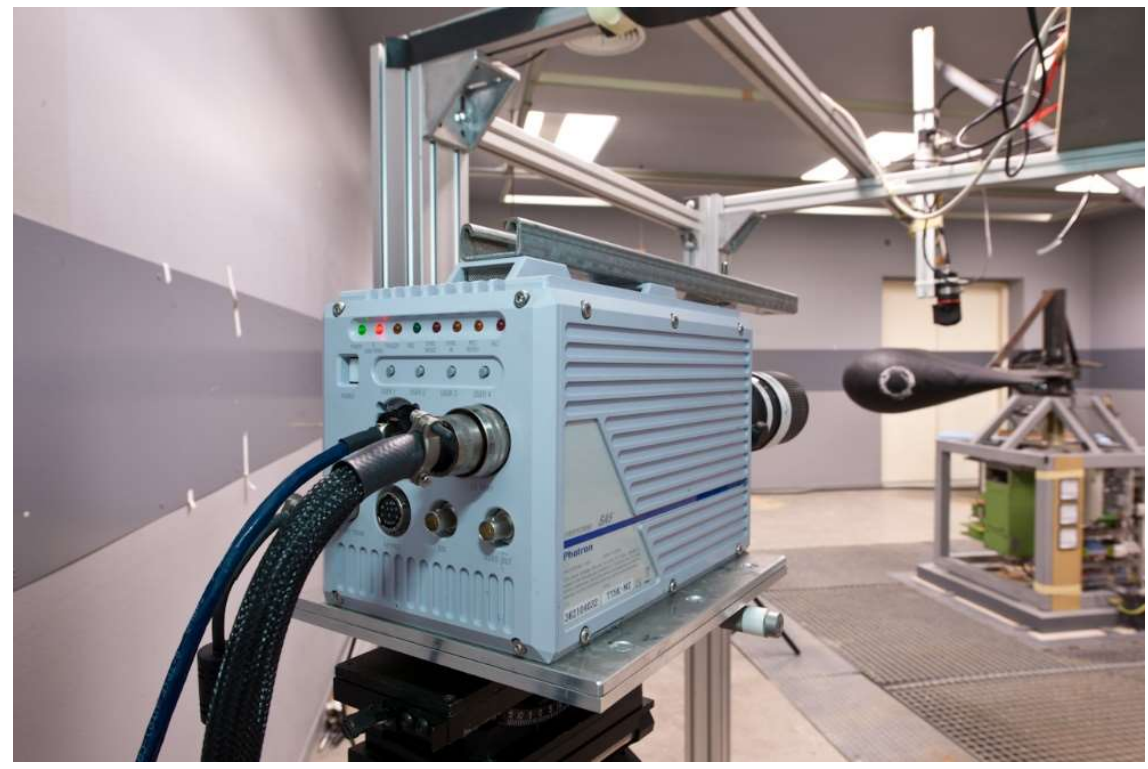

Figure 2.2.5.1. High speed camera.

The optical camera was a Photron SA-5 with a capturing range from $10^{3}$ frames per second (fps) to $10^{6} \mathrm{fps}$. In the present campaign, the capturing rate was $75000 \mathrm{fps}$ with a resolution of 192 x 312 pixels. Also, extra lenses were used for additional magnification of the images: $200 \mathrm{~mm}$ micro Nikkor lens with a 2x doubler located in between the camera and the $200 \mathrm{~mm}$ lens and extension rings.

\subsection{FLOW CHARACTERIZATION.}

Characterisation of the flow field was performed by using a Particle Image Velocimetry system (PIV). Specifically, it was a TSI System with illumination provided by two pulsed Nd-Yag $190 \mathrm{~mJ}$ lasers. A Power View Plus 4MP camera was used to record the images with a resolution of 2048 x 2048 pixels. Camera lenses were AF-S VR Micro Nikkor 105 $\mathrm{mm} \mathrm{f/2.8} \mathrm{G} \mathrm{IF-ED} \mathrm{Nano} \mathrm{Crystal} \mathrm{Coat,} \mathrm{AF} \mathrm{Nikkor} \mathrm{80-200} \mathrm{mm} \mathrm{f/2.8} \mathrm{D} \mathrm{IF-ED,} \mathrm{and} \mathrm{Nikkor}$ $50 \mathrm{~mm} \mathrm{f} / 1.4$. Particles used to sow the flow were olive oil droplets having a diameter of 1 $\mu \mathrm{m}$. The TSI Insight $3 \mathrm{G}$ software was used for synchronized of image capturing, flow illumination and the subsequent analysis. Sampling of the flow field was carried out at frequencies in the range from $4.75 \mathrm{~Hz}$ to $6.5 \mathrm{~Hz}$. Time between two consecutive laser pulses varied between $1.1 \mu \mathrm{s}$ and $200 \mu \mathrm{s}$. At $34 \mu$ (the time used for the fastest moving airfoil) particle moved about $3 \mathrm{~mm}$ that is a distance much smaller than the characteristic length of the problem that is the airfoil leading edge radius $(103 \mathrm{~mm}, 70 \mathrm{~mm}$ and $30 \mathrm{~mm}$ respectively). PIV interrogation areas were divided into smaller sub-interrogation areas for analysis purposes. The spatial flow field resolution in the test windows near the airfoil leading edges was of the order of $1 \mathrm{~mm}$. Additional details on the specifics of the PIV analysis can be found in Sor and García-Magariño 2011 and García-Magariño et al 2015. 


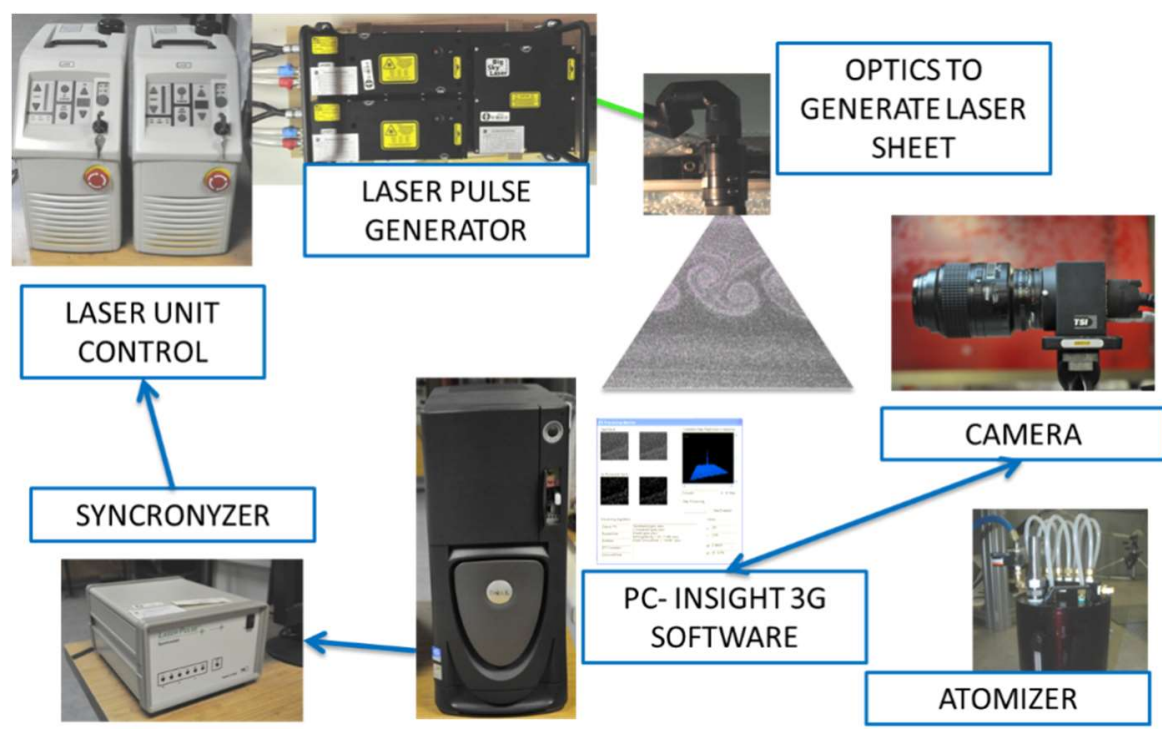

Figure 2.3.1. PIV System.

The flow field velocity and acceleration time histories were characterized in the absence of falling water droplets. This means that, implicitly, a one way interaction approach was considered (Iuliano et al 2011). The methodology used to obtain the different velocity fields in the airfoil reference frame out of a series of consecutive PIV standing frames is described in Sor and García-Magariño al 2011. 15 different flow velocities and acceleration profiles were used in the experimental campaign. This corresponds to three airfoil models (M1, M2 and M3) and five translational velocities for each of them (see table 2.2.2.2). In practice, from the droplet trajectory model point of view, this means that 15 different flow velocity and acceleration profiles could be used for testing purposes. To give an impression of the range that was actually covered, the stagnation stream line histories of flow velocity at the extremes of the velocity range $(50 \mathrm{~m} / \mathrm{s}$ to $90 \mathrm{~m} / \mathrm{s})$ for the three airfoil models are presented in figure 2.3.2. The acceleration histories for these cases are presented in figure 2.2.3. 


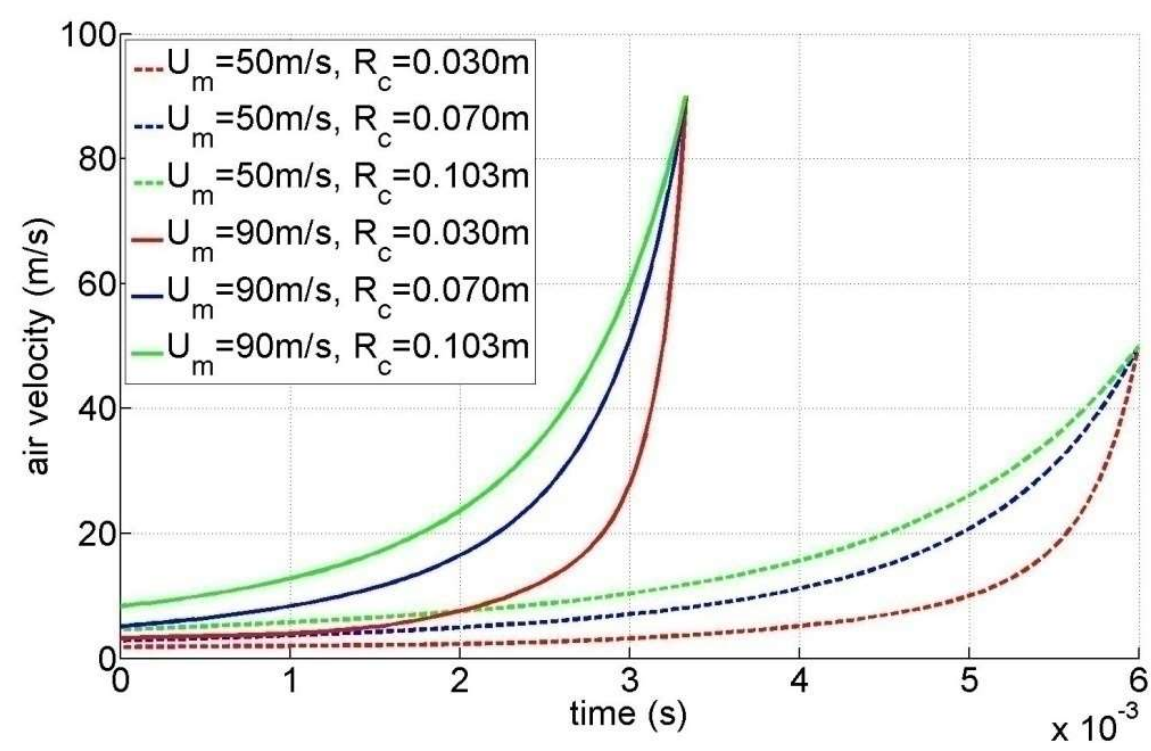

Figure 2.3.2. Velocity time histories at the stagnation streamline for thee airfoil models (M1, M2 and M3) at translational velocities of $50 \mathrm{~m} / \mathrm{s}$ and $90 \mathrm{~m} / \mathrm{s}$.

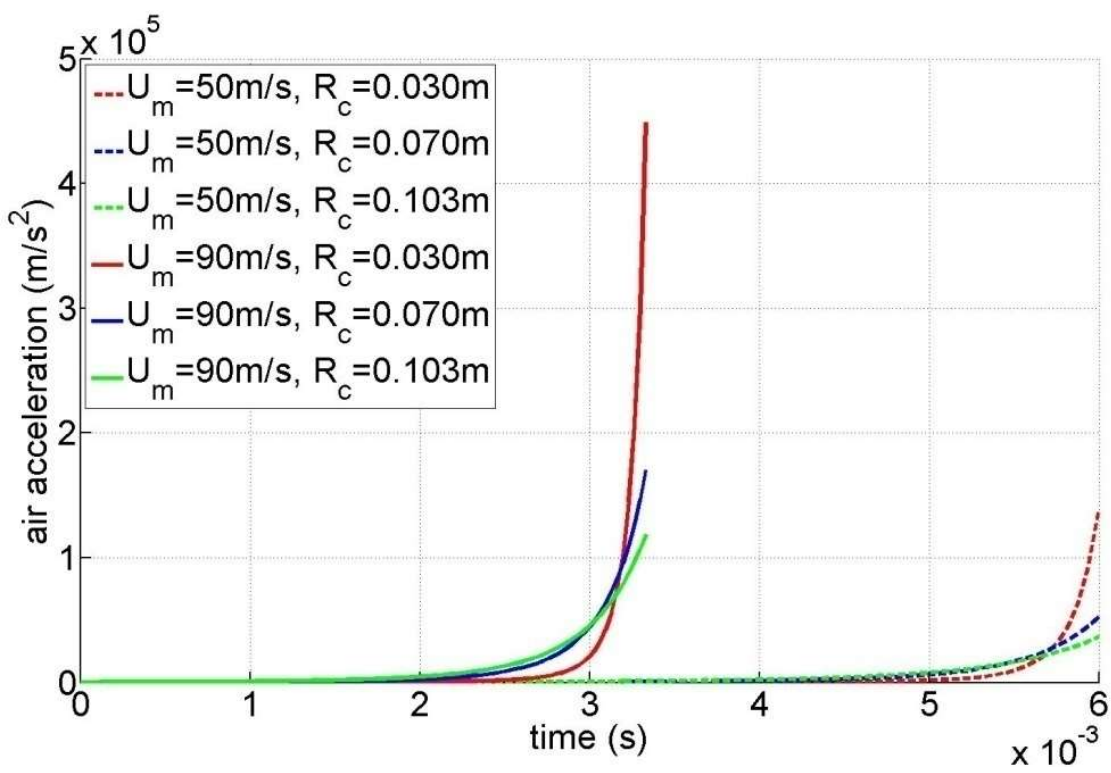

Figure 2.3.3. Acceleration time histories at the stagnation streamline for thee airfoil models (M1, M2 and M3) at translational velocities of $50 \mathrm{~m} / \mathrm{s}$ and $90 \mathrm{~m} / \mathrm{s}$.

Figure 2.3.4 presents the three uncertainty bands associated to the 3 limiting velocity profiles corresponding to $90 \mathrm{~m} / \mathrm{s}$ of maximum airfoil velocity as presented in figure 2.3.2. These bands were obtained using all recorded velocity profiles to calculate the standard deviation and plotting this standard deviation band. The typical spread was of the order of $\pm 0.8 \mathrm{~m} / \mathrm{s}$ that for an average velocity in the range between $20 \mathrm{~m} / \mathrm{s}$ and $40 \mathrm{~m} / \mathrm{s}$ (see figure 2.3.4) yields and uncertainty of the order of $\pm 2 \%$ to $\pm 4 \%$. Figure 2.3 .5 shows the kernel density estimate of the measurement at time $3 \mathrm{~ms}$ the case of intermediate airfoil (M2) at the maximum velocity $(90 \mathrm{~m} / \mathrm{s})$. 


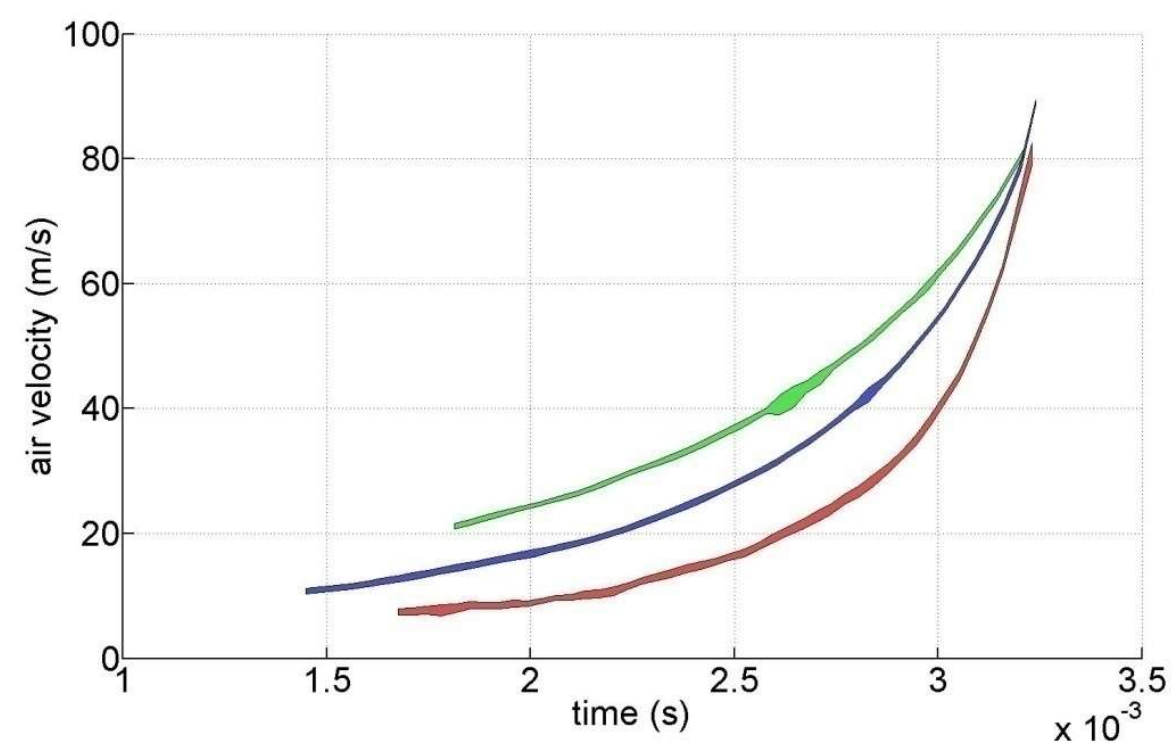

Figure 2.3.4. Uncertainty bands associated to the 3 limiting velocity profiles ( $\mathrm{Um}=90$ $\mathrm{m} / \mathrm{s}$ ) presented in figure 2.3.2.

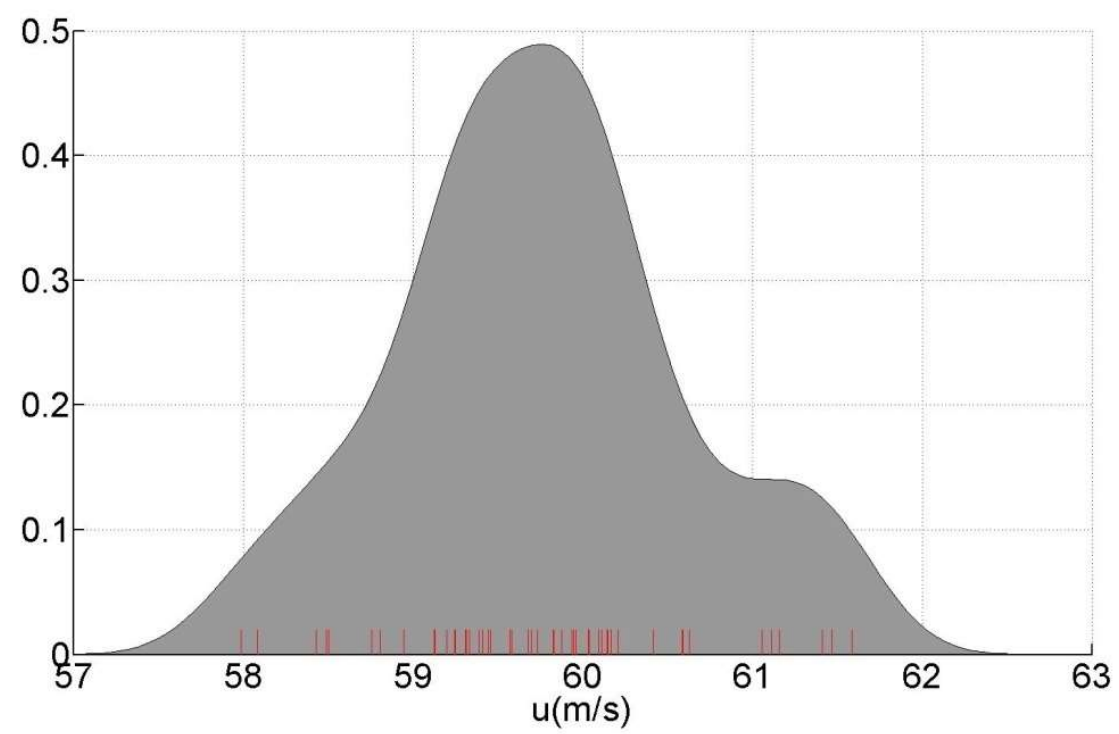

Figure 2.3.5. Kernel density estimate of the measurement at time $3 \mathrm{~ms}$ the case of intermediate airfoil (M2) at the maximum velocity $(90 \mathrm{~m} / \mathrm{s})$.

Figure 2.3.6 shows the $2 \mathrm{D}$ velocity map in the vertical plane, in the airfoil reference frame, obtained for the airfoil velocity of $90 \mathrm{~m} / \mathrm{s}$. Figure 2.3 .7 shows the equivalent 2D velocity map in the horizontal plane along the span wise, showing that in the center of the model the span wise velocity component is negligible. These results are the average of 50 different measurements. It could be argued that flow characterization could have been done far more easily using an analytical approach; for example, using the potential flow 
velocity profile past an airfoil. However it was decided to do it experimentally because of three different facts:

a) The flow field was turbulent (the Reynolds number was in the range from $2,000,000$ to $5,000,000$ ).

b) The airfoil trajectory was circular (instead of rectilinear) in a closed room where the flow was continuously being disturbed.

c) The airfoil was not two dimensional but, instead, it had a finite length in the span wise direction.

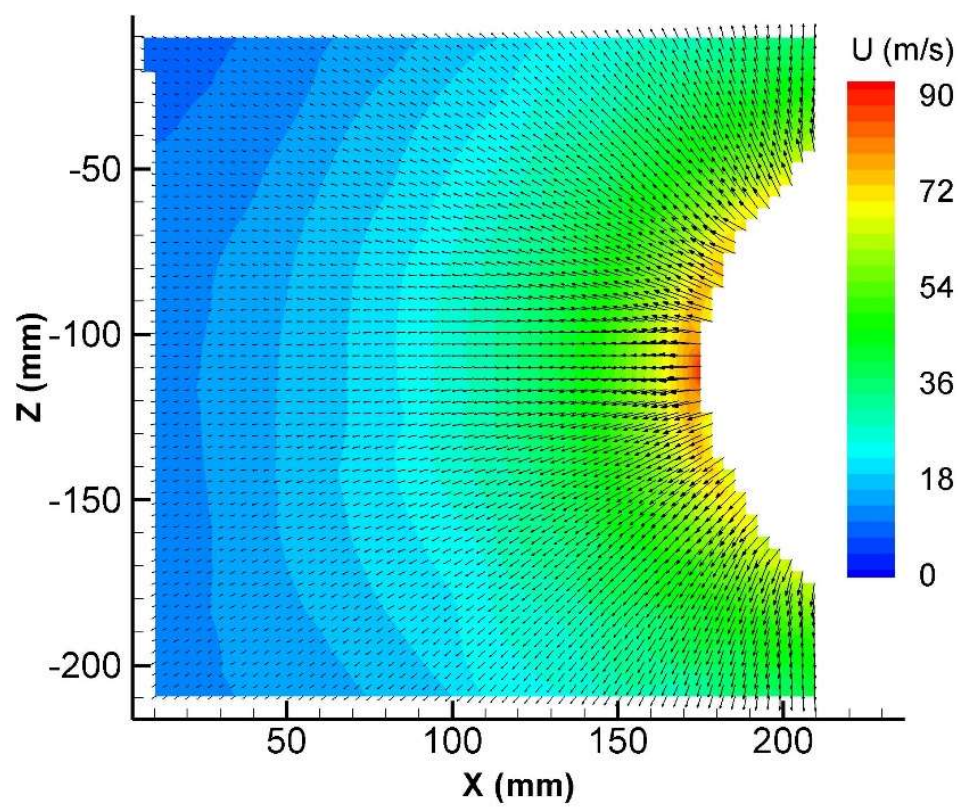

Figure 2.3.6. Average flow field velocity in the vertical plane for the 50 measurements. The airfoil velocity was $90 \mathrm{~m} / \mathrm{s}$.

With regard to repeatability, each case was repeated 50 times. It was found that dispersion of the results was larger the higher the airfoil velocity. Figure 2.3.8 shows the 2D map of the Standard Deviation of the 50 measurements at the highest airfoil velocity. In this case, the spread was of the order of $\pm 3 \mathrm{~m} / \mathrm{s}$ that is much smaller than the airfoil velocity ( 90 $\mathrm{m} / \mathrm{s})$. 


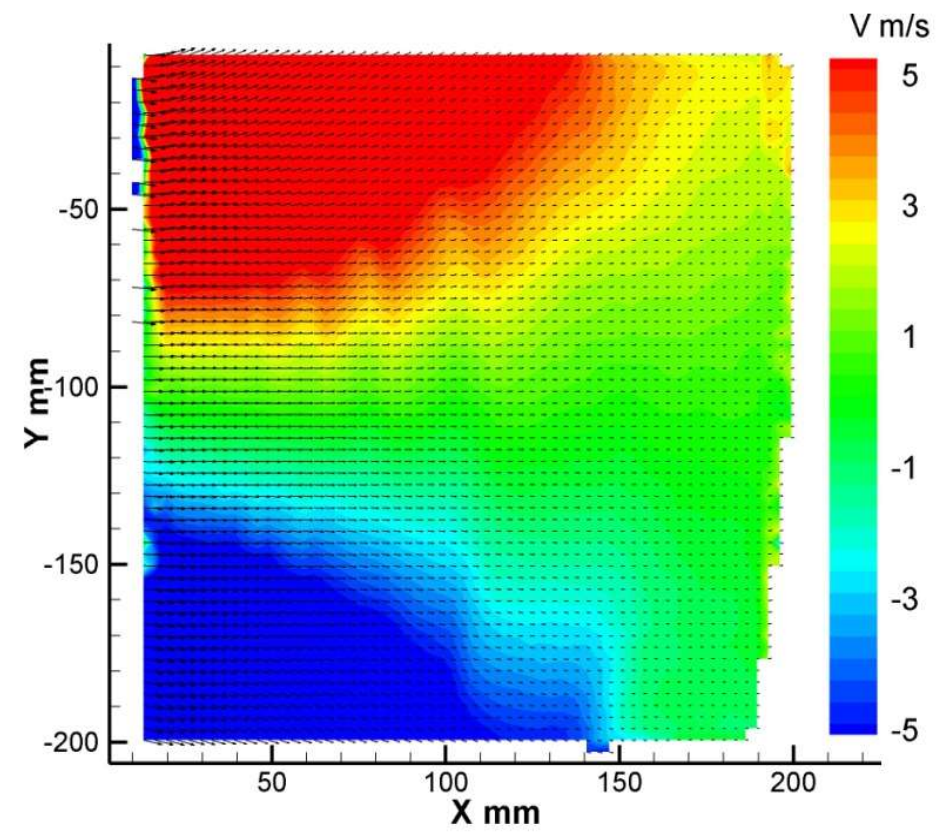

Figure 2.3.7. Average flow field velocity in the horizontal plane for the 50 measurements. The airfoil velocity was $90 \mathrm{~m} / \mathrm{s}$.

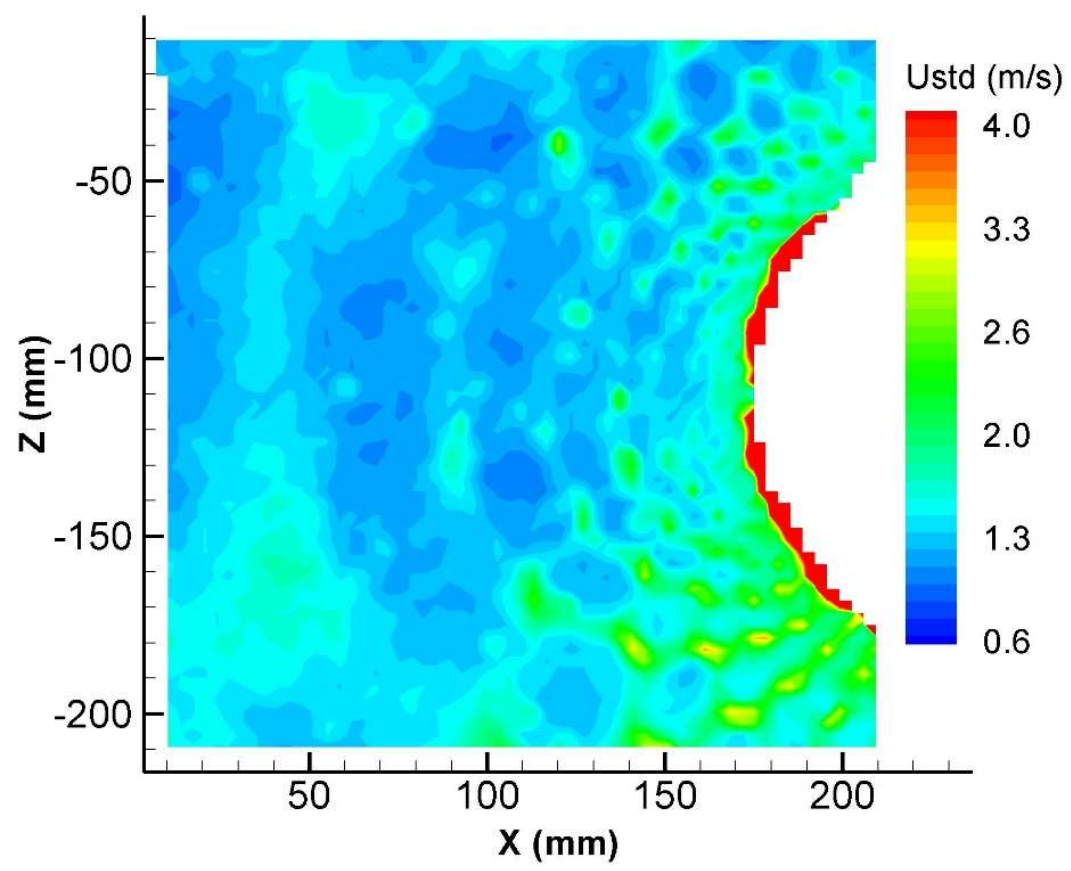

Figure 2.3.8. 2D map of the Standard Deviation of the 50 flow field measurements for airfoil velocity equal to $90 \mathrm{~m} / \mathrm{s}$. 


\subsection{TESTS MATRIX.}

Two experimental campaigns were conducted: the first one was in the stagnation line of the airfoil model while during the second campaign droplets were in the shoulder of the airfoil model leading edge.

The summary of the 45 tests cases addressed in the first experimental campaign in the stagnation line is provided below.

- Three droplet diameters $\left(R_{d}\right): 575 \mu \mathrm{m}, 775 \mu \mathrm{m}$, and $1025 \mu \mathrm{m}$.

- Three airfoils leading edge radius $\left(R_{c}\right): 0.103 \mathrm{~m}, 0.070 \mathrm{~m}, 0.030 \mathrm{~m}$.

- Five airfoil velocities $\left(U_{m}\right): 50 \mathrm{~m} / \mathrm{s}, 60 \mathrm{~m} / \mathrm{s}, 70 \mathrm{~m} / \mathrm{s}, 80 \mathrm{~m} / \mathrm{s}$, and $90 \mathrm{~m} / \mathrm{s}$.

Regarding the uncertainty associated to the measurement of droplet trajectories, figure 2.4.1 shows an example of three different measurements of the case with $R_{c}=0.070 \mathrm{~m}$, $R_{d}=388 \mu \mathrm{m}$, and $U_{m}=71 \mathrm{~m} / \mathrm{s}$.

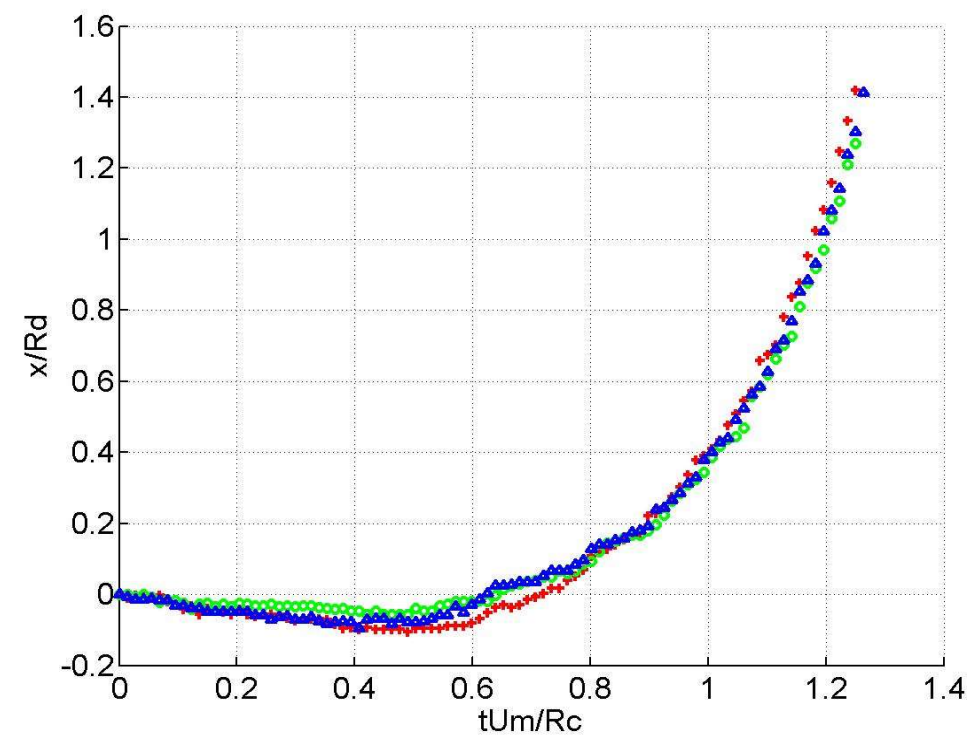

Figure 2.4.1. Results corresponding to three different measurements of the droplet trajectory of the same experimental case: $R_{c}=0.070 \mathrm{~m}, R_{d}=388 \mu \mathrm{m}$, and $U_{m}=$ $71 \mathrm{~m} / \mathrm{s}$.

Nine different cases were addressed in the second experimental campaign conducted in the shoulder region of the leading edge of the airfoil model M2 (see table 2.2.2.2). They corresponded to the variation of two parameters:

- Parameter \#1: the velocity of the approaching airfoil. Three cases were considered: $50 \mathrm{~m} / \mathrm{s}, 70 \mathrm{~m} / \mathrm{s}$ and $90 \mathrm{~m} / \mathrm{s}$. 
- Parameter \# 2: the position relative to the airfoil of the field of vision (FOV) where the droplet deformation was recorded. Three different heights of the mid-line of the FOV with respect to the airfoil stagnation line were considered: FOV \#1: 38 $\mathrm{mm}$, FOV \#2: $58 \mathrm{~mm}$, and FOV \#3: $66 \mathrm{~mm}$.

The initial droplet diameter (of the order of $1 \mathrm{~mm}$ ) and the airfoil model were unchanged through the experiments. To ensure repeatability, each of the nine experimental cases was repeated four times.

\subsection{EXPERIMENTAL DATA PROCCESING.}

An specific Matlab application called SITEA was developed for quick processing purposes due to the large amount of frames images (Sor et al 2012). The application track each droplet selected giving the trajectory, and the maximum, minimum and equivalent droplet diameters. In addition the software calculates velocities, accelerations and the dimensionless instantaneous parameters (Reynolds, Bond, and Weber numbers...) along the stagnation line. All the tests data obtained were classified to be input to the application. A storage format was defined to perform a later study.

\subsubsection{PRE-PROCESSING.}

For each configuration test, several clips of images were recorded. Pre-processing consists of two steps:

1. The conversion from the video format to a format compatible with Matlab, PNG in this case.

2. The generation of an Excel file that identifies and contains all the information related to each clip of images to be analyzed by the application (test configuration, initial and final frame identification, indentifying the frame in which model appears, location and generic name of the files...)

\subsubsection{PROCESSING. MATLAB APPLICATION.}

The processing starts with the first frame of each clip with the following steps: 
1.- Selection of the droplet to be tracked by marking on the frame a region of interest that includes only that droplet.

2.- A threshold is computed using Otsu's Method (Otsu 1979) that converts the image to a binary image.

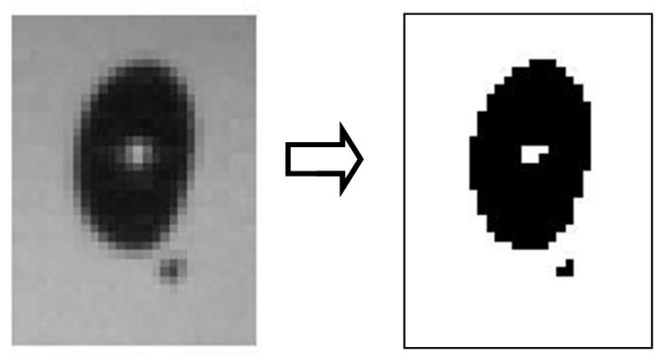

Figure 2.5.2.1. Region of interest converted to binary image.

The contrast of the image is an important issue since it could modify the droplet contour in the binary image. Figure 2.5.2.2 shows an example, of two images of the same glass sphere taken at two illumination and consequently two contrast levels, yielding different binary images (Sor and García-Magariño 2012).
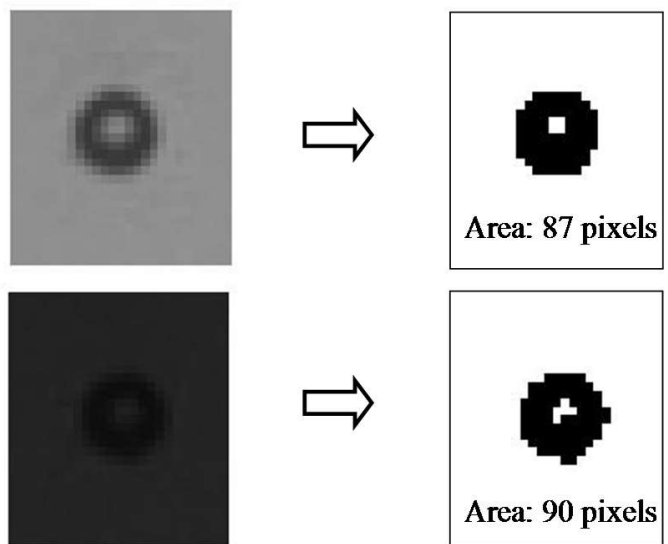

Figure 2.5.2.2. Shadow images of a glass sphere with different illumination. Influence of the image contrast on the binary image conversion.

3.- A filtering process is conducted in three steps:

a) Image conversion to negative. 


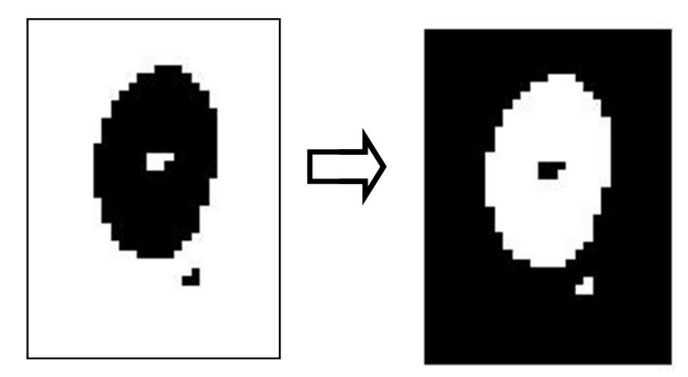

Figure 2.5.2.3. Binary image converted to negative.

b) Dust cleaning. White objects or dots owning areas smaller than $10 \%$ of the expected droplet area are eliminated using the command "bwareaopen".
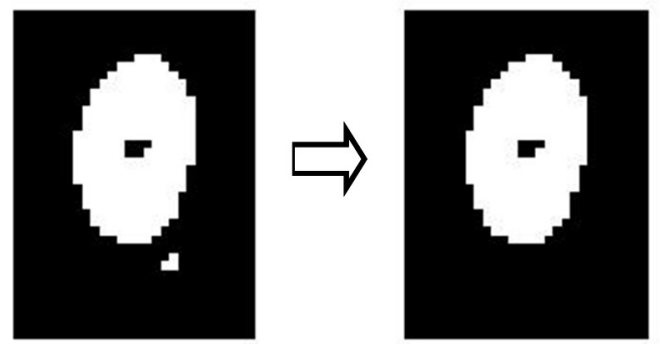

Figure 2.5.2.4. Cleaning dust.

c) Glint elimination. Due to light glints, white holes may appear in the centre of the droplet. This hole is removed using the command "imfill".
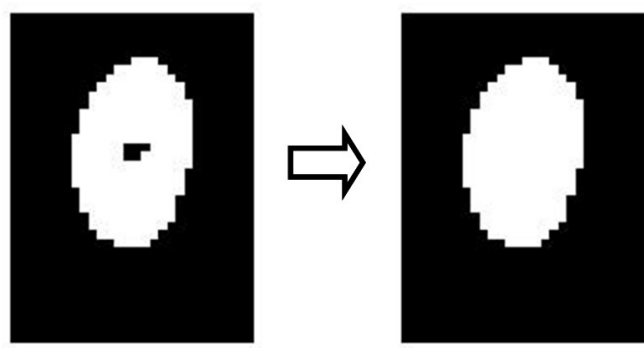

Figure 2.5.2.5. Glints elimination.

4.- The centroid position in the region of interest and the principal properties (droplet area, the minimum and the maximum diameter) are obtained using the command "regionprops". The actual diameter is calculated on the assumption that droplets become two semi-spheroids joined together during the deformation (see figure 2.5.2.6). As observed in figure 2.5.2.7, this assumption seems to be very accurately. In this figure the maximum, the minimum, the equivalent diameter as obtained by Matlab (which is the 2D equivalent diameter), and the real droplet diameter on the assumption of an oblate spheroid are plotted. It can be observed 
that the droplet diameter remains constant up to a point where the minimum diameter experiences a minimum, instant at which the droplet breaks up.

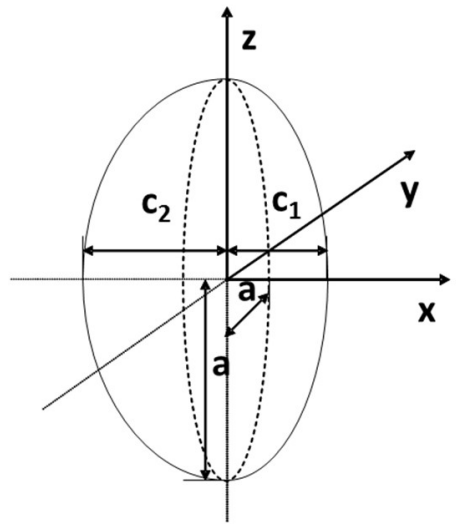

$$
\begin{gathered}
D_{\text {min }}=c_{1}+c_{2} \\
D_{\text {max }}=2 \cdot a \\
D=\sqrt[3]{D_{\text {max }}^{2} \cdot D_{\text {min }}}
\end{gathered}
$$

Figure 2.5.2.6. Droplet shape assumption during deformation.

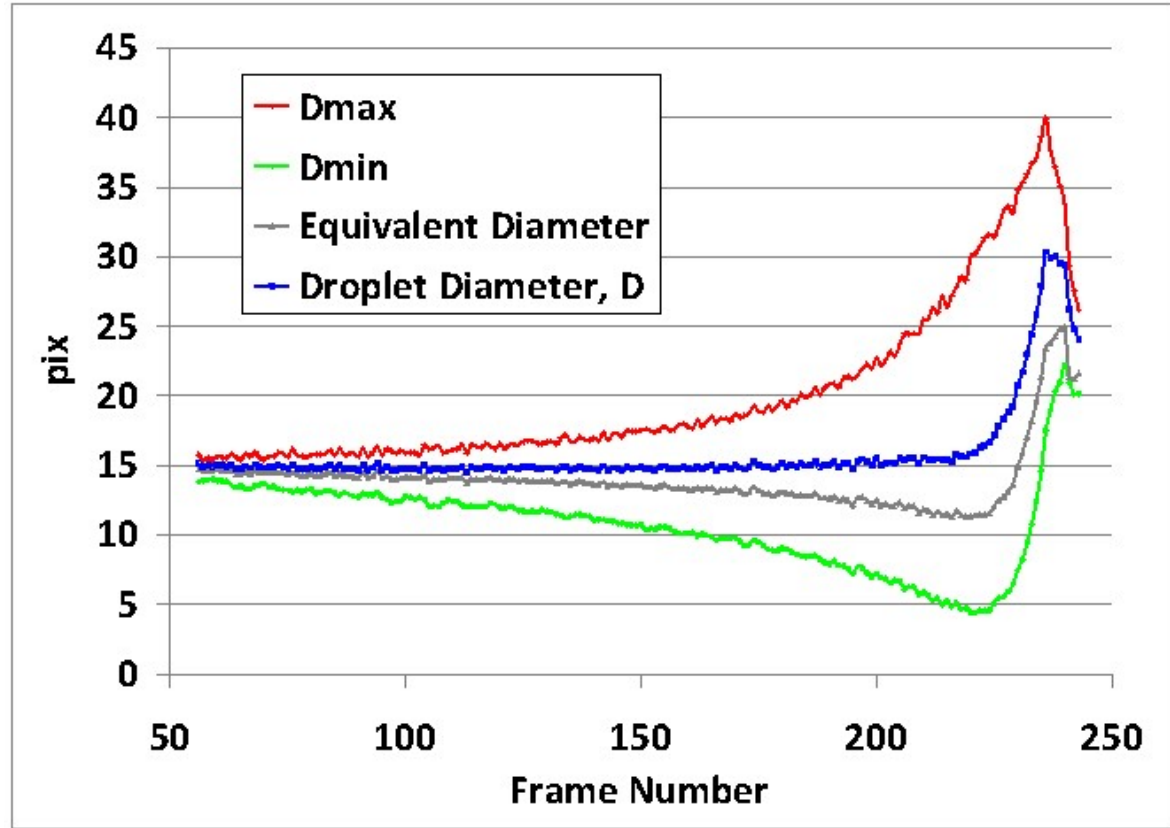

Figure 2.5.2.7. Evolution of the maximum, minimum, 2D equivalent and droplet diameter.

The magnification was previously calibration and it is used to convert pixel in the international system measurements.

To continue the droplet tracking, the same region of interest is chosen in the next frame, but centered in the centroid of the previous frame. Each frame is processed in a similar way as the first frame. The velocity of the model and the rate of frame acquisition are constant, so the droplet positions in the model reference system is defined by equation. (2.1), where $f_{m}$ is the frame number where the leading edge position is measured, $f_{d i}$ is the number of the frame where the droplet position is measured, $U_{m}$ is the model velocity, 
$F R$ is the rate of frame adquisition, $x_{m f}$ is the leading edge position in $\mathrm{fm}, x_{d i}$ is the droplet position in $f_{d i}, \mathrm{Mg}$ is the camera magnification.

$$
X_{d i}=\frac{\left(f_{m}-f_{d i}\right)}{F R} U_{m}+\frac{\left(x_{m f}-x_{d i}\right)}{M g}
$$

Droplet evolution is additionally characterized by the acceleration along the trajectory and the instantaneous dimensionless parameters: Reynolds, Weber, Bond, and the Drag Coefficient, appearing all defined in equations (2.2)-(2.5). $U_{r d}$ is the slip velocity, D is the droplet diameter, $\rho_{a}$ is the air density, $\rho_{d}$ is the droplet velocity, and $\sigma_{d}$ is the water surface tension for the droplet (Vargas et al 2010).

$$
\begin{gathered}
\operatorname{Re}=\frac{\rho_{a} U_{r d} D}{\mu_{a}} \\
W e=\frac{\rho_{a} U_{r d}^{2} D}{\sigma_{w}} \\
B o=\frac{\rho_{w} D^{2}}{\sigma_{w}}\left(\frac{d U_{r d}}{d t}\right) \\
C_{d}=\frac{4}{3} \frac{\rho_{w}}{\rho_{a}} \frac{D}{U_{r d}} \frac{d U_{r d}}{d t}
\end{gathered}
$$

The slip velocity can be calculated from droplet and air velocities. The flow field in front of the model is measured in advance with PIV technique (Sor and García-Magariño 2011). Droplet velocity and acceleration are calculated from a fit curve of droplet positions.

SITEA additionally gives the droplet breakup instant. This instant is obtained by minimizing the fitted function of the droplet minimum diameter evolution following the breakup criteria used in Vargas et al (2010).

\subsubsection{DATA STORAGE.}

The correct storage of a large amount of data is of vital importance for a later postprocessing of results. Two options were considered to save the data: an excel file or a Matlab variable structure. The excel file has the advantage of simplicity of use and access whereas Matlab permits greater capacity for post-processing. The post-processing requirements of this application made the Matlab structure the selected option. However both of them were used leading to a redundancy useful for checking purposes.

\subsubsection{VALIDATION.}


In order to validate SITEA, several cases were selected. The evolution of the droplets was tracked by SITEA and by Spotlight program. Spotlight program is a program developed by NASA addressed to other applications that also tracks the position of droplets. Using Spotlight and calculating manually the rest of parameters, excellent agreement between the two applications was obtained. The development of SITEA embodies additional features to the single droplet trajectory calculation given by Spotlight.

\subsubsection{COMPUTATIONAL TIME.}

The computational time was about a $10 \%$ of the pre-processing time. Nevertheless, the total analysis time was reduced in a $90 \%$ compared to the other analysis method. A basic computer (Pentium IV, 2800Mhz; 2Gb RAM) was used, needing 20 seconds to process 250 frames.

\subsubsection{EXAMPLE OF APPLICATION.}

This section presents an example of application of the method. Once the input panel is filled in with the data of the selected case, the application starts asking for a region of interest. The tracking is shown on the screen for checking purposes.
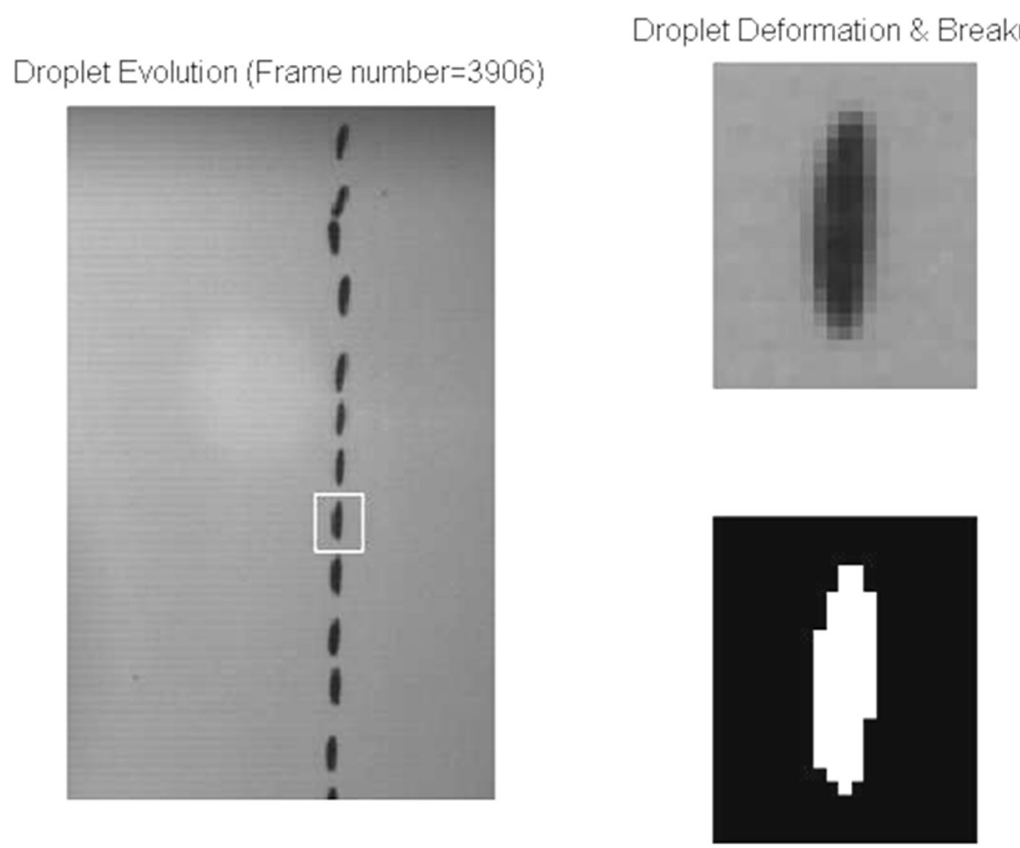

Figure 2.5.6.1. Window showing the tracking evolution of the droplet. On the left each frame with the region of interest marked is shown. On the right the region of interest is shown before and after the image processing.

Once the tracking has finished, an estimate of the horizontal position evolution is shown in a plot. 


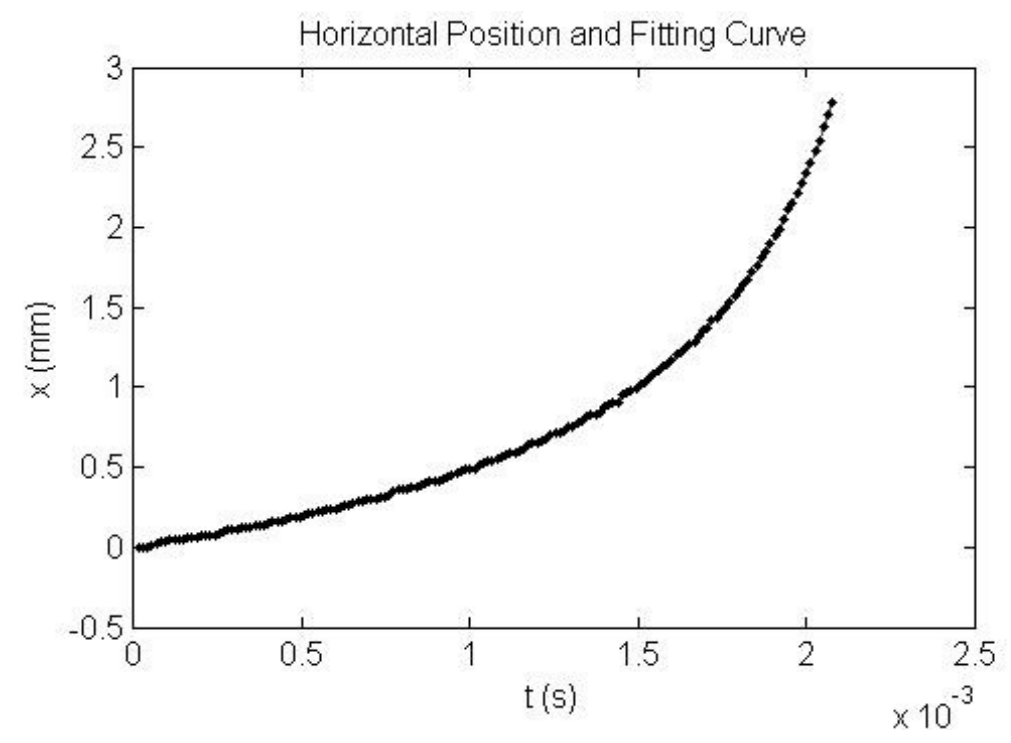

Figure 2.5.6.2. Window showing tracking evolution and fitting curve of the water droplet.

The criterion used for the experimental determination of breakup consists of the finding of the minimum value of the curve of minimum diameter. Accordingly the minimum diameter is approximated by a curve that allows the determination of the minimum. The evolution of the minimum diameter together with the approximation curve is shown in a new plot for checking purposes.

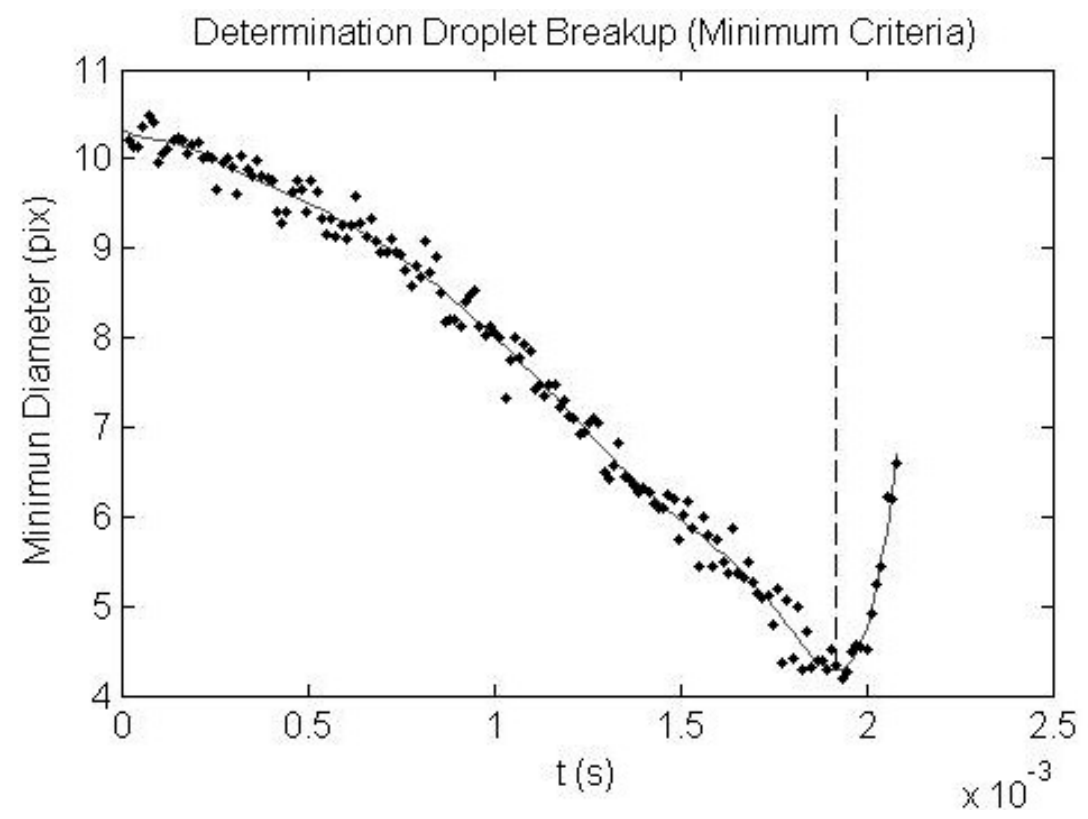

Figure 2.5.6.3. Window showing tracking evolution of the droplet minimum diameter and fitted curve.

The evolution of the droplet trajectory and different shaped parameters are finally displayed for each drop in another figure. 

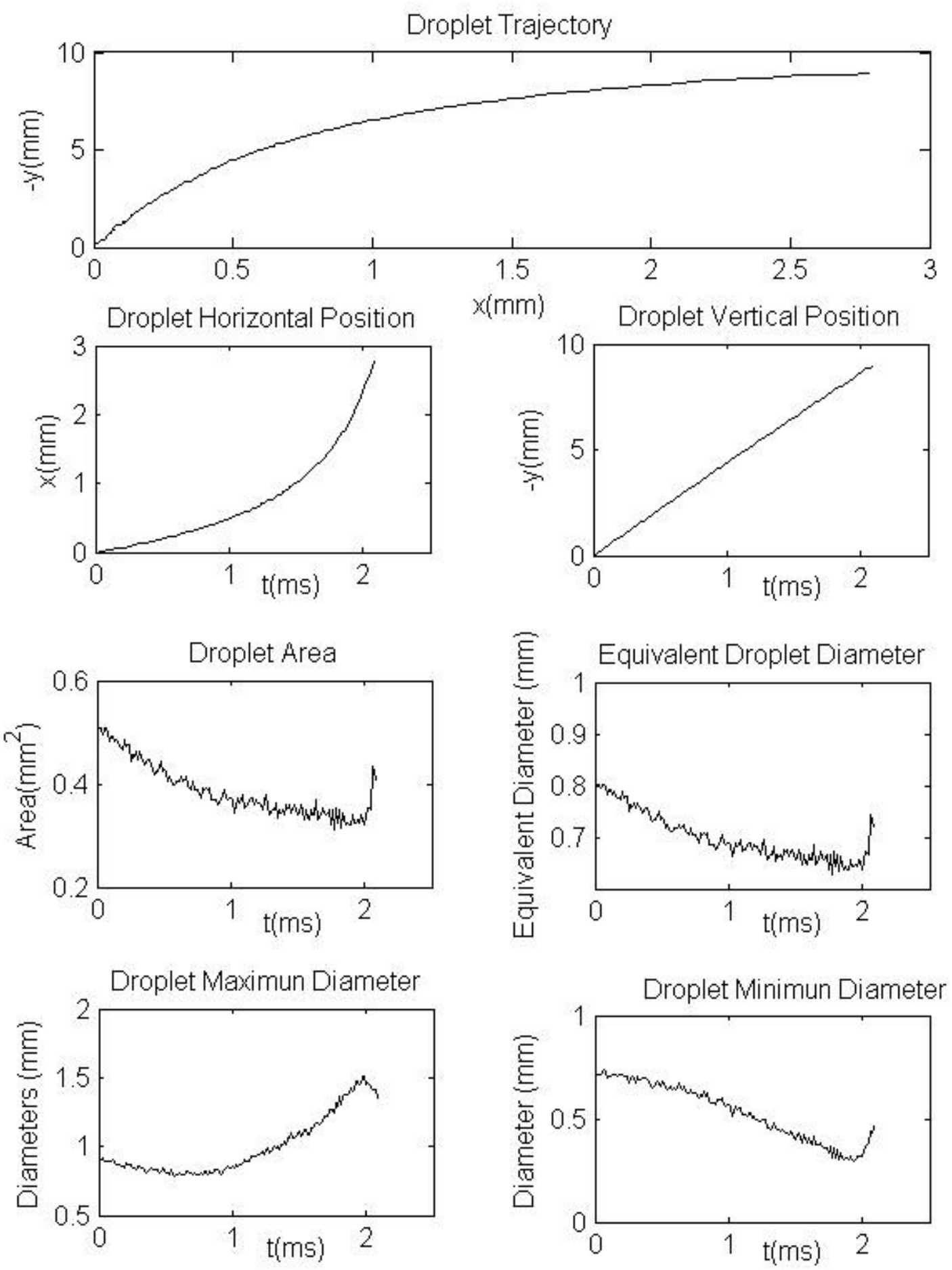

Figure 2.5.6.4. Window showing the summary of the droplet evolution.

Once the evolution of the tracking has been examined in the summary figure, the data are saved including all the evolution of the dimensionless parameters that are shown in a separate figure. 

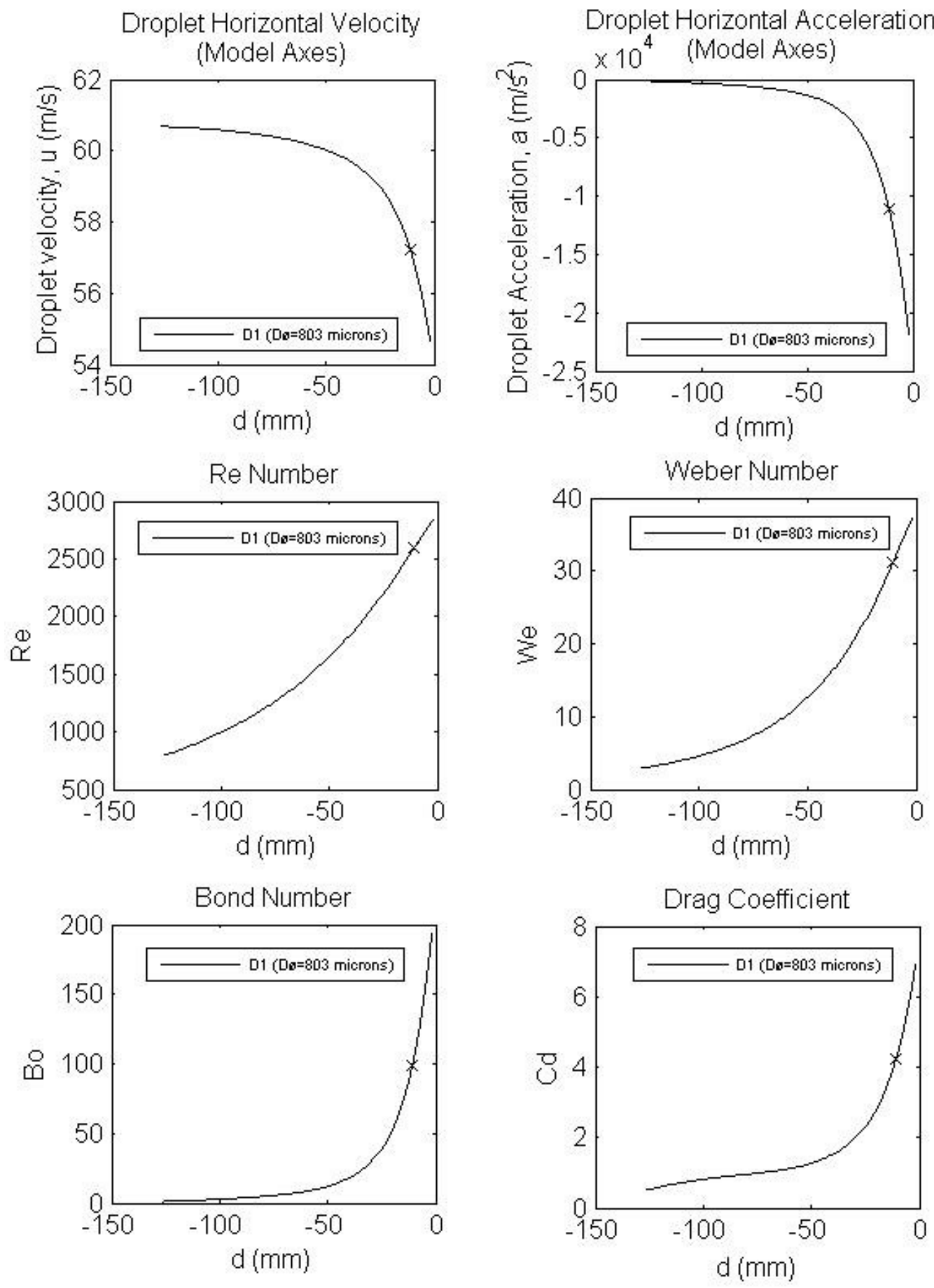

Figure 2.5.6.5. Window showing a summary of all the calculated data. 


\section{DROPLET TRAJECTORY AND DEFORMATION MODEL IN THE STAGNATION LINE OF AN AIRFOIL.}

A theoretical model is presented to predict water droplet trajectories in the flow past an airfoil. The model accounts for droplet deformation and includes a drag coefficient that considers the influence of flow acceleration. To validate the theoretical model, a series of experimental tests have been carried out in a rotating arm facility. Comparison between the results obtained after computation of the model differential equations and those recorded in the experiments showed a good agreement. This suggests that, within the range of parameters that has been tested, the theoretical model could be confidently used for trajectory prediction purposes.

The work presented in this chapter and the next chapter has been submitted, accepted and published in Aerospace Science and Technology (Sor et al 2016).

\subsection{MODEL DESCRIPTION.}

The three model equations are:

$$
\begin{gathered}
m_{d} \frac{d^{2} x}{d t^{2}}=F_{d r a g_{-} x} \\
m_{d} \frac{d^{2} y}{d t^{2}}=-F_{d r a g_{-} y}+m_{d} g \\
\frac{3 m_{d}}{16} \frac{d^{2} a}{d t^{2}}=+F_{v}+F_{s t}+F_{P}
\end{gathered}
$$

where:

- $x, y, a$, and $t$ are the coordinates of the droplet centre of mass, the halfmaximum dimension of the deformed droplet, and time respectively. 
- $m_{d}$ is the droplet mass equal to $4 \pi \rho_{d} R_{d}^{3} / 3\left(R_{d}\right.$ is the initial droplet radius and $\rho_{d}$ is the droplet density) and $g$ is the gravity acceleration.

- $F_{\text {drag } x}, F_{d r a g_{-} y}, F_{v}, F_{s t}$, and $F_{P}$ are the "x" and "y" components of the aerodynamics drag force, the viscous force and surface tension force that oppose droplet deformation, and the flow pressure force that acts on the droplet surface respectively.

\subsection{HYPOTHESES.}

It is assumed that the droplet motion is governed by three equations: two dynamics equations (3.1-3.2) that represent the equilibrium of forces in the horizontal (x) and vertical (y) directions, and one equation (3.3) that models the droplet deformation. This equation (3.3) influences the droplet drag force because, indirectly, it allows for the computation of the droplet cross-section area normal to the incoming flow. Figure 3.2.1 shows a sketch of the acting forces and the coordinates axis. The coordinates' axes (fixed in space) are located in the droplet centre of mass at the precise moment when it enters the measurement window.

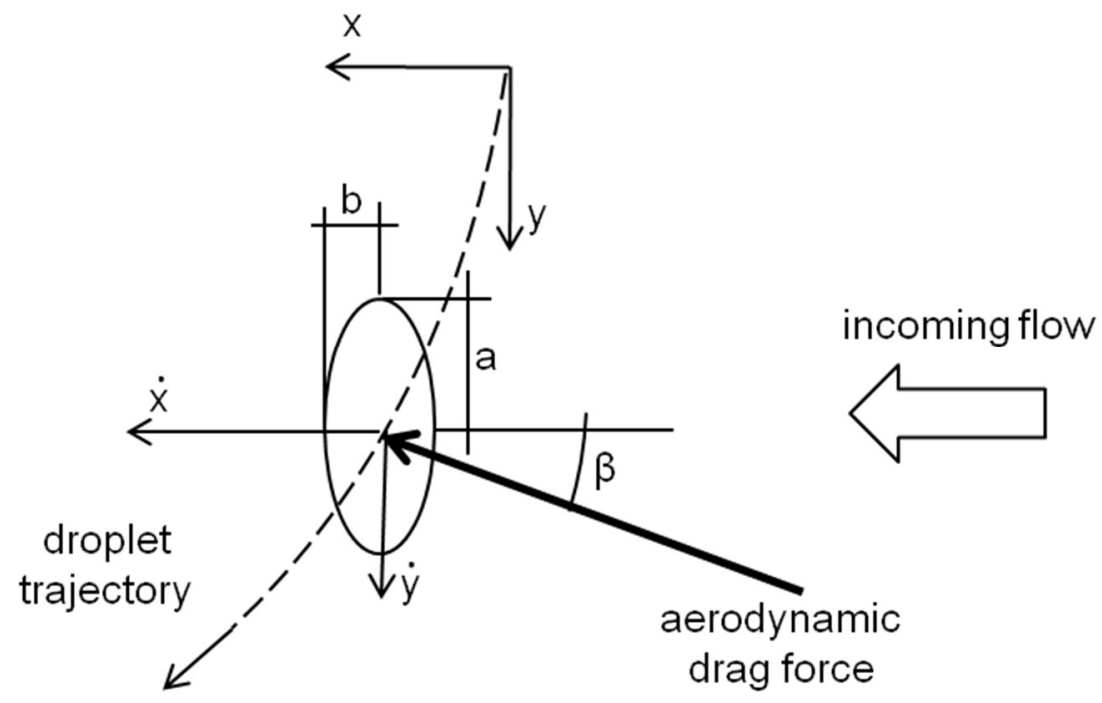

Figure 3.2.1. Sketch of the problem under consideration.

Apart from some other considerations, the model presented is based on three main hypothesis that have been verified analyzing the experimental data obtained during the completion of the experimental campaigns. These hypothesis are: 
- The "y" component of the incoming airflow $\left|V_{\text {air } \_} y\right|$ is very small $\left(V_{\text {air }} y \cong\right.$ 0 ). This assumption (that also implies that $\left|V_{\text {air_ }}\right| \gg\left|V_{\text {air }_{-} y}\right|$ ) means that the model is valid, formally, in the vicinity of the stagnation streamline (stagnation region) of the incoming airfoil only.

- It is assumed that the droplet deforms as an oblate spheroid.

- Because of the first hypothesis, the slip velocity in the horizontal direction is much larger than the slip velocity in the vertical direction (this is, of course, not true during the initial instants of the droplet trajectory but velocities are very low at these stages and droplet deformation is negligible). Then, it is further hypothesized that the droplet deforms, only, along the vertical direction that is perpendicular to the direction of the much larger horizontal slip velocity. The practical implication of this third hypothesis is that forcing terms in the equation that models droplet deformation (equation 3.3) depend, only, on the horizontal slip velocity; thereby decoupling, effectively, the " $\mathrm{x}$ " equation of motion (equation 3.1) and the equation for droplet deformation (equation 3.3) from the "y" equation of motion (equation 3.2).

\subsubsection{DRAG MODEL.}

The aerodynamic drag forces $F_{d r a g_{-} x}$ and $F_{d r a g_{-}} y$ depend on the slip velocities $V_{s x}$ and $V_{s y}$ defined as:

$$
V_{s x}=V_{a i r_{-} x}-\frac{d x}{d t}, \quad V_{s y}=\frac{d y}{d t}
$$

It has been assumed previously that $V_{s x} \gg V_{s y}$. Then, if $\beta$ is the angle between the total drag force and the "x" axis, the following approximations hold: $\sin \beta \cong V_{s y} / V_{s x}$ and $\cos \beta \cong 1$. Then, $F_{\text {drag_x }}$ in equation (3.1) can be modeled as:

$$
F_{\text {drag_ } x} \cong \frac{1}{2} \rho_{\text {air }} V_{s x}^{2} \pi a^{2} C_{D}
$$

where $\rho_{\text {air }}$ is the air density and $C_{D}$ is the drag coefficient. Now, the modeling of this drag coefficient is the most critical aspect of the formulation presented in this section. 
Specifically, it was decided that $C_{D}=C_{D 1}+C_{D 2}$, where $C_{D 1}$ and $C_{D 2}$ model steady and unsteady effects respectively as follows:

$$
\begin{gathered}
C_{D 1}=C_{D_{\text {sphere }}}^{b / a} \cdot C_{D_{\text {disk }}}^{1-b / a} \\
C_{D 2}=k \frac{b}{V_{s x}^{2}} \frac{d V_{s x}}{d t}
\end{gathered}
$$

where $C_{D s p h e r e}$ is the known drag coefficient of a perfect sphere based on a Reynolds number defined using the instantaneous (time dependent) slip velocity as the characteristic velocity, and the instantaneous (time dependent) droplet maximum elongation: "2a" (see figure 3.2.1), as the characteristic length. $C_{\text {Ddisk }}$ is the drag coefficient of a disk whose value, assumed to be constant in the range of Reynolds numbers considered, is taken to be 1.17. In practice, relation (3.6) assumes that the quasi steady part of the droplet drag coefficient is obtained by interpolating between the drag of a perfect sphere and the drag of a disk. The parameter that controls the interpolation is the droplet aspect ratio $b / a$, see figure 3.2.1. Since the droplet has constant known volume, $V_{d}$, the values of " $a$ " (obtained from equation 3.3) and " $b$ " are related by a simple geometry relation: $V_{d}=(4 \pi / 3) a^{2} b$ that means $R_{d}^{3}=a^{2} b$. Relation (3.7) assumes that the unsteady part of the droplet drag coefficient depends on the functional $\left(1 / V_{s x}^{2}\right) d V_{s x} / d t$ that is directly related to the acceleration parameter as defined by Temkin and Metha (1982). The main difference here with the formulation of Temkin and Metha (1982) is that, in the present model, $C_{D 2}$ depends, also, on the instantaneous (time dependent) droplet elongation " $b$ " in the "x" direction. $k$ is a calibration coefficient that in the range of experimental cases that have been tested (that will be described in the next chapter 4) has been found to be constant and equal to 9 .

Within the same set of hypothesis, $F_{d r a g_{-} y}$ in equation (3.2) can be approximated as:

$$
F_{\text {drag } \_} \cong \frac{1}{2} \rho_{a i r} V_{s x} V_{s y} \pi a^{2} C_{D}
$$




\subsubsection{DEFORMATION MODEL.}

The equation (3.3) correspond to a droplet deformation model previously derived and discussed (Sor and Garcia-Magariño 2015), which is shown in an appendix at the end of this chapter.

The three terms in the right hand side of equation (3.3) are modeled as follows:

$$
F_{v}=-\frac{64}{9} \mu_{d} \pi R_{d}^{3} \frac{1}{a^{2}} \frac{d a}{d t}
$$

where $\mu_{d}$ is the droplet viscosity. This term represents the force that viscosity opposes to droplet deformation. It has been included into equation (3.3) for the sake of completion. However, for the range of parameters considered in the experimental tests, its actual value was found to be much smaller than the other two terms in the right hand side of equation (3.3). If a different fluid is chosen for the tests, for instance: glycol that has much higher viscosity than water, this term becomes comparable to the other two terms. Then, since this model will deal with water droplets only, this viscous term will be dropped altogether from the formulation.

$$
F_{s t}=-\frac{4}{3} \sigma \frac{d A_{s}}{d a}
$$

This surface tension term $F_{s t}$ represents the force that surface tension opposes to droplet deformation. $\sigma$ is the water surface tension and $A_{s}$ is the surface area of the droplet. $d A_{s} / d a$ represents the rate of variation of droplet surface area with respect to the maximum elongation for a constant volume droplet. Since the deformed droplet is assumed to have the shape of an oblate spheroid, the derivative $d A_{S} / d a$ can be obtained by purely geometrical considerations. In particular:

$$
\begin{aligned}
& \frac{d A_{s}}{d a}=4 \pi a-\frac{4 \pi R_{d}}{\varepsilon\left(a / R_{d}\right)^{5}} \ln \left(\frac{1+\varepsilon}{1-\varepsilon}\right)+ \\
& +\frac{3 \pi R_{d}}{\varepsilon\left(a / R_{d}\right)^{11}}\left[\frac{2}{\varepsilon\left(1-\varepsilon^{2}\right)}-\frac{1}{\varepsilon} \ln \left(\frac{1+\varepsilon}{1-\varepsilon}\right)\right]
\end{aligned}
$$


where $\varepsilon=\left[1-(b / a)^{2}\right]^{1 / 2}$. Note that $d A_{s} / d a$ is a known functional that depends on the variable $a$ only. For the reminder of the thesis this functional will be referred to as $F(a)$.

The last term in equation (3.3) represents the pressure force that the incoming flow exerts on the droplet. The actual effect of this term is to promote deformation.

$$
F_{P}=\frac{1}{2} c_{p} \rho_{a i r} V_{s x}^{2} \pi R_{d}^{2}
$$

where $c_{p}$ is a proportional coefficient that has been calibrated and that has a constant value of 0.93 . The calibration is shown in the appendix at the end of this chapter. This pressure term should be the integral of a local pressure times a differential surface area and, therefore, it cannot be computed as such since the droplet surface local pressure distribution is unknown. This is the reason why relation (3.12) represents a global approximation to that integral. It could be argued that $F_{P}$ in relation (3.12) should scale with $a^{2}$ instead of $R_{d}^{2}\left(a>R_{d}\right)$. However, as discussed by Ibrahim et al. (1993), the fact that pressure decreases sharply up from the droplet stagnation streamline region down to the droplet edge region suggests that scaling with a parameter smaller than $a$ might be more appropriate. Note that within the hypothesis of this model, relation (3.12) involves $V_{s x}$ only and not $V_{s y}$ that means, as mentioned earlier, that equations (3.1) and (3.3) are effectively decoupled from equation (3.2).

\subsubsection{INITIAL CONDITIONS.}

The initial conditions for equations (3.1-3.3) are:

$$
\begin{aligned}
& x(0)=0, \quad\left(\frac{d x}{d t}\right)_{t=0}=0 \\
& y(0)=0, \quad\left(\frac{d y}{d t}\right)_{t=0}=V_{0} \\
& a(0)=R_{d}, \quad\left(\frac{d a}{d t}\right)_{t=0}=0
\end{aligned}
$$


where $V_{0}$ is the droplet falling velocity when it enters the test window. In an actual generic case, it should be taken equal to the terminal falling velocity of the droplet.

\subsection{DIMENSIONLESS MODEL EQUATIONS.}

Now, it is convenient to re-write equations (3.1-3.3) in dimensionless form. To this end, the following dimensionless variables and parameters are defined:

$$
\begin{gathered}
\eta=\frac{x}{R_{d}}, \quad \zeta=\frac{y}{R_{d}}, \quad \alpha=\frac{a}{R_{d}}, \quad \tau=\frac{t U_{m}}{R_{c}} \\
\mathbb{V}_{s x}=\frac{V_{s x}}{U_{m}}, \quad \mathbb{V}_{s y}=\frac{V_{s y}}{U_{m}}, \quad \mathbb{A}_{d}=\frac{A_{s}}{R_{d}^{2}}, \quad \mathbb{F}(\alpha)=\frac{F(a)}{R_{d}}
\end{gathered}
$$

where $U_{m}$ is the airfoil velocity and $R_{c}$ its radius of curvature. Within this new change of coordinates, the model equations and initial conditions are written as:

$$
\begin{gathered}
\frac{d^{2} \eta}{d \tau^{2}}=\Pi_{1} \mathbb{V}_{s x}^{2} \alpha^{2}\left[\left(C_{D_{\text {sphere }}}^{\left(R_{d} / a\right)^{3}} \cdot C_{D_{\text {disk }}}^{1-\left(R_{d} / a\right)^{3}}\right)+\left(\Pi_{2} \frac{1}{\alpha^{2} \mathbb{V}_{s x}^{2}} \frac{\mathrm{d} \mathbb{V}_{s x}}{\mathrm{~d} \tau}\right)\right] \\
\frac{d^{2} \zeta}{d \tau^{2}}=-\Pi_{1} \alpha^{2} \mathbb{V}_{s x} \mathbb{V}_{s y}\left(C_{D_{\text {sphere }}}^{\left(R_{d} / a\right)^{3}} \cdot C_{D_{\text {disk }}}^{1-\left(R_{d} / a\right)^{3}}\right)+\Pi_{3} \\
\frac{d^{2} \alpha}{d \tau^{2}}=-\Pi_{4} \mathbb{F}(\alpha)+\frac{16}{3} \Pi_{1} C_{P} \mathbb{V}_{s x}^{2}
\end{gathered}
$$

where:

$$
\Pi_{1}=\left[\frac{3}{8} \frac{\rho_{\text {air }}}{\rho_{d}}\left(\frac{R_{c}}{R_{d}}\right)^{2}\right]
$$




$$
\begin{gathered}
\Pi_{2}=\frac{k R_{d}}{R_{c}} \\
\Pi_{3}=\frac{g R_{c}}{U_{m}^{2}}\left(\frac{R_{c}}{R_{d}}\right) \\
\Pi_{4}=\frac{16}{3 \pi} \frac{\sigma}{\rho_{d}}\left(\frac{R_{c}}{U_{m} R_{d}}\right)^{2} \frac{1}{R_{d}}
\end{gathered}
$$

The dimensionless initial conditions are:

$$
\begin{gathered}
\eta(0)=0, \quad\left(\frac{d \eta}{d \tau}\right)_{\tau=0}=0 \\
\zeta(0)=0, \quad\left(\frac{d \zeta}{d \tau}\right)_{\tau=0}=\frac{R_{c}}{R_{d}} \frac{V_{0}}{U_{m}} \\
\alpha(0)=1, \quad\left(\frac{d \alpha}{d \tau}\right)_{\tau=0}=0
\end{gathered}
$$

Numerical integration of equations (3.18) and (3.20) with initial conditions (3.25) and (3.27) is straightforward. Then, after $x(t)$ and $a(t)$ have been obtained $b(t)$ is easily computed and equation (3.19) together with initial condition (3.26) can be integrated. In the experimental test cases described in the next section, $V_{0}$ in initial condition (3.26) was close to the terminal falling velocity of the droplets which means that the droplet vertical velocity in the test window was nearly constant. In fact, the tracking equipment was not able to detect significant changes in this vertical velocity. 


\section{APPENDIX: DEFORMATION EQUATION.}

This appendix shows the necessity of a new equation for the droplet deformation in a continuously accelerated flow. Traditional deformation model based of the analogy between a droplet and a spring mass system are first described and applied to this type of flows showing the necessity of a new deformation equation. Following the line of thinking of the previous deformation models a new droplet deformation equation is obtained (the equation (3.3) of the model proposed in this thesis). For simplicity, in this appendix, this equation will be referred as DRD (Droplet Ratio Deformation), in order to distinguish from the previous deformation models. The proportional coefficient $c_{p}$ of equation (3.12) is obtained from the experimental data. The final droplet deformation equation is validated using 159 experimental cases that cover five model velocities, three model sizes and droplet diameters between $0.3 \mathrm{~mm}$ and $1.4 \mathrm{~mm}$.

The work presented in this Appendix has been submitted, accepted and published in Journal of Aircraft (Sor et al 2015).

\section{A.1. PREVIOUS DEFORMATION MODELS.}

The three main droplet deformation and breakup models based on the analogy between a droplet and a spring mass system (see figure A.1.1) are TAB (O'Rourke and Amsden 1987), Clark's (Clark 1988) and DDB models (Ibrahim et al 1993). These three models have been widely compared with experimental data where droplets are suddenly exposed to an air stream. The following sections describe briefly these models.

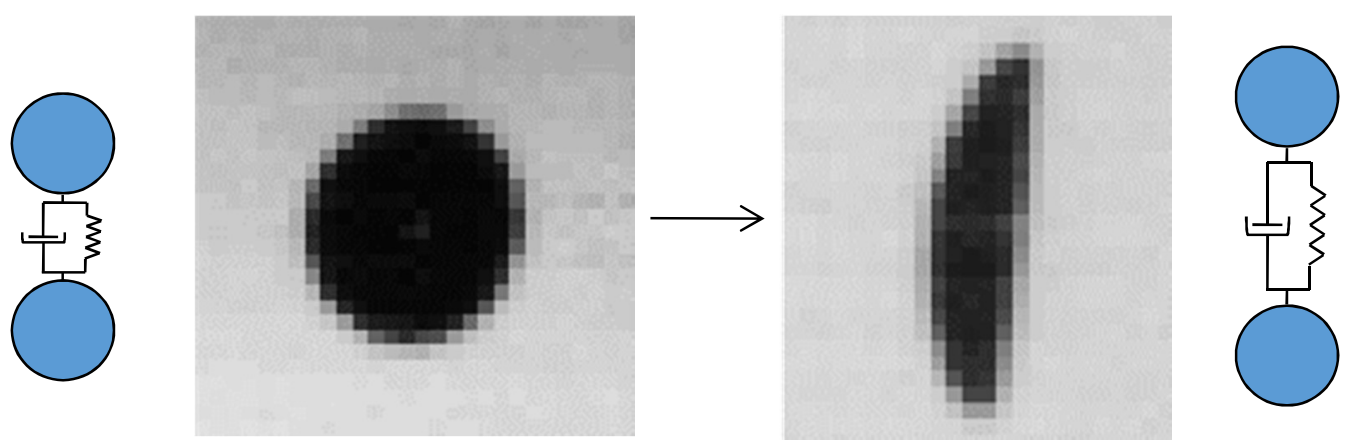

Figure A.1.1 Analogy between a droplet and a mass-spring system.

\section{A.1.1. TAB Model.}

Taylor suggested the analogy between an oscillation and distorting droplet, and a springmass system. The equation of a damped, forced harmonic oscillator is given by 


$$
s_{0} \frac{d^{2} x}{d t^{2}}+s_{1} \frac{d x}{d t}+s_{2} x=F
$$

The spring force is analogous to the surface tension forces, whereas the external force on the mass is analogous to the aerodynamic force. Liquid viscosity is taken into account in the damping forces. According to O'Rourke and Amsden (1987) the equation of the TAB model can be written as

$$
K \frac{d^{2} y^{\prime}}{d t^{\prime 2}}+\frac{5 N}{R e} \frac{d y^{\prime}}{d t^{\prime}}+\frac{8}{W e} y^{\prime}=\frac{2}{3}
$$

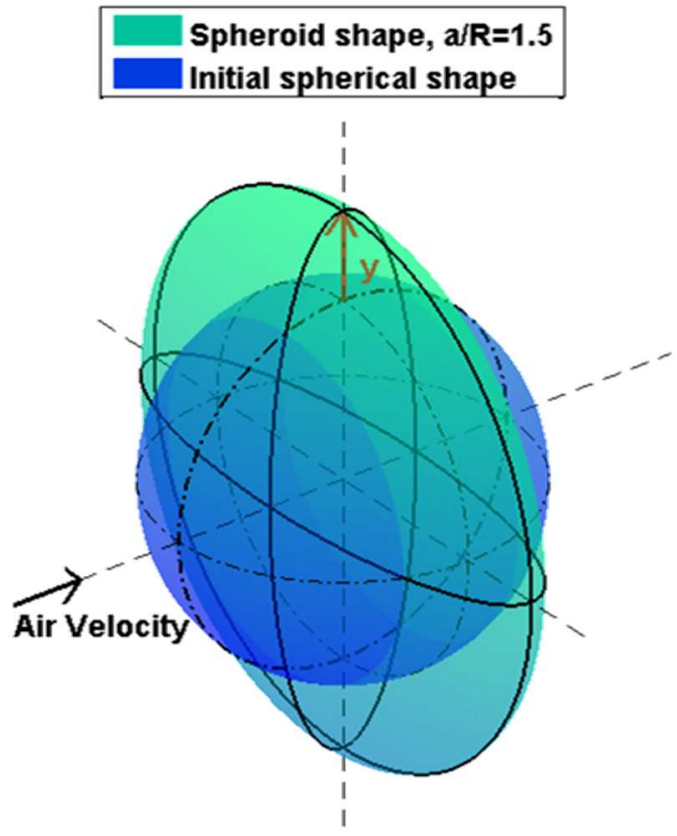

Figure A.1.1.1. TAB model

where $K$ is the densities liquid-gas ratio $K=\rho_{l} / \rho_{g}, N$ is the viscosities ratio $N=\mu_{l} / \mu_{g}$, $R e$ is the Reynolds Number $R e=\rho_{g} U_{\infty} R_{d} / \mu_{g}, W e$ is the Weber Number $W e=$ $\rho_{g} U_{\infty}^{2} R_{d} / \sigma, t^{\prime}$ is the dimensionless time $t^{\prime}=t U_{\infty} / R_{d}$ and $y^{\prime}$ is the dimensionless displacement of the equator of the droplet from its equilibrium position (see Fig A.1.1.1).

\section{A.1.2. Clark Model.}

Clark's model (Clark 1988) again proposes an analogy between the oscillating and distorting droplet, and a spring-mass system. Two masses in the mechanical model are considered concentrated at the centers of mass of the halves of the drop. Forces acting upon one half of the drop are evaluated by an approximated analytical approach. The final equation follows: 


$$
(K+1) \frac{d^{2} y^{\prime}}{d t^{\prime 2}}+\frac{9 \pi^{2}(N+1)}{4 R e} \frac{d y^{\prime}}{d t^{\prime}}+\frac{9 \pi^{2}}{4 W e}\left(y^{\prime}-\frac{4}{3 \pi}\right)=\frac{2}{\pi}
$$

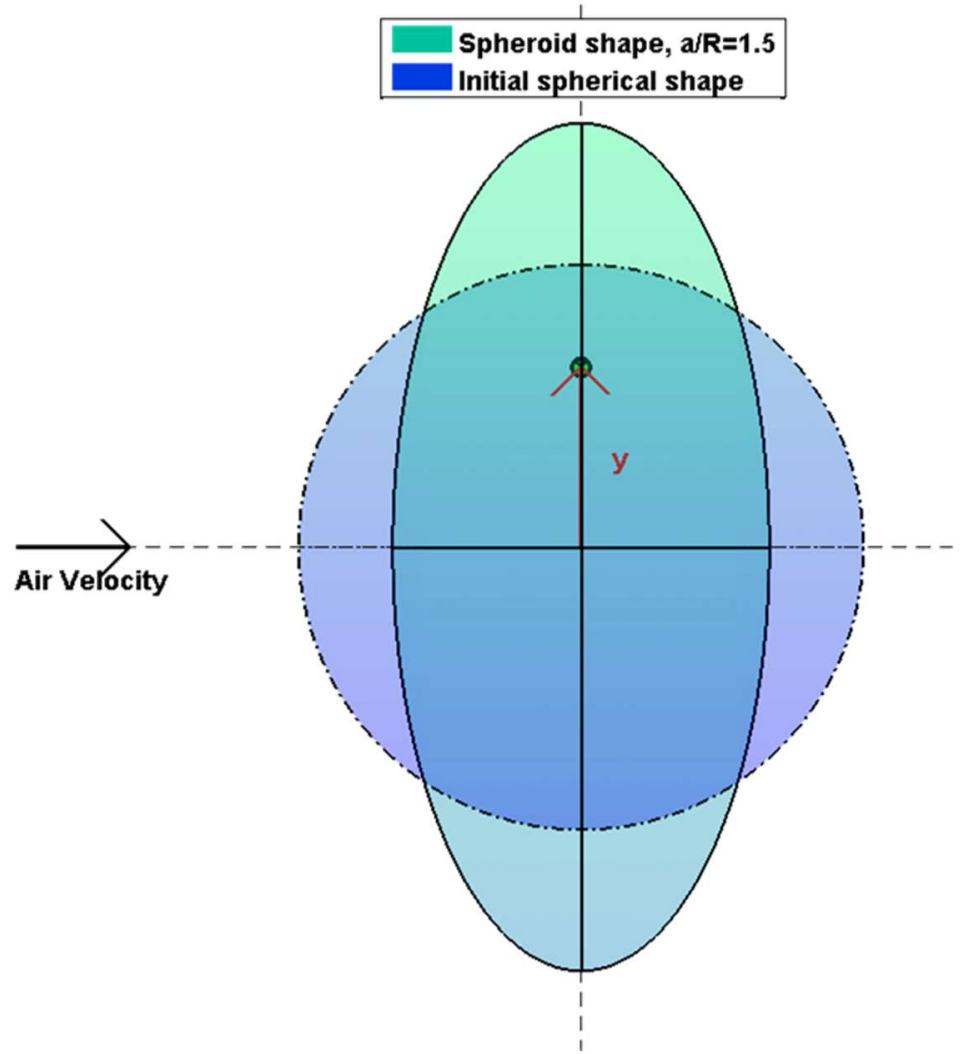

Figure A.1.2.1. Clark Model.

In equation (3.30) $y^{\prime}$ is the dimensionless distance between the center of mass of the half drop and the center of the drop (see figure A.1.2.1). Clark's model employs an approximate linearized analytical approach.

\section{A.1.3. DDB Model.}

The Droplet Deformation and Breakup model (Ibrahim et al 1993) assumes that the liquid drop is deformed due to a pure extensional flow from an initial spherical shape of radius $R$ into an oblate spheroid with an ellipsoidal cross section of half-major axis a and halfminor axis b. Drop dynamics are formulated in terms of the motion of the center of mass of the half drop (see figure A.1.3.1). The problem is addressed by the energy equation, with no heat exchange with its surroundings. The internal energy comes from the kinetic and the potential energy due to the surface tension of the droplet. The work is done by pressure and viscous forces. The resultant equation for this model is

$$
K \frac{d^{2} y^{\prime}}{d t^{\prime 2}}+\frac{4 N}{R e} \frac{1}{y^{\prime 2}} \frac{d y^{\prime}}{d t^{\prime}}+\frac{3 c^{2}}{W e}\left(1-2\left(c y^{\prime}\right)^{-6}\right)=\frac{3}{8}
$$


where $c=a / y=3 \pi / 4$.

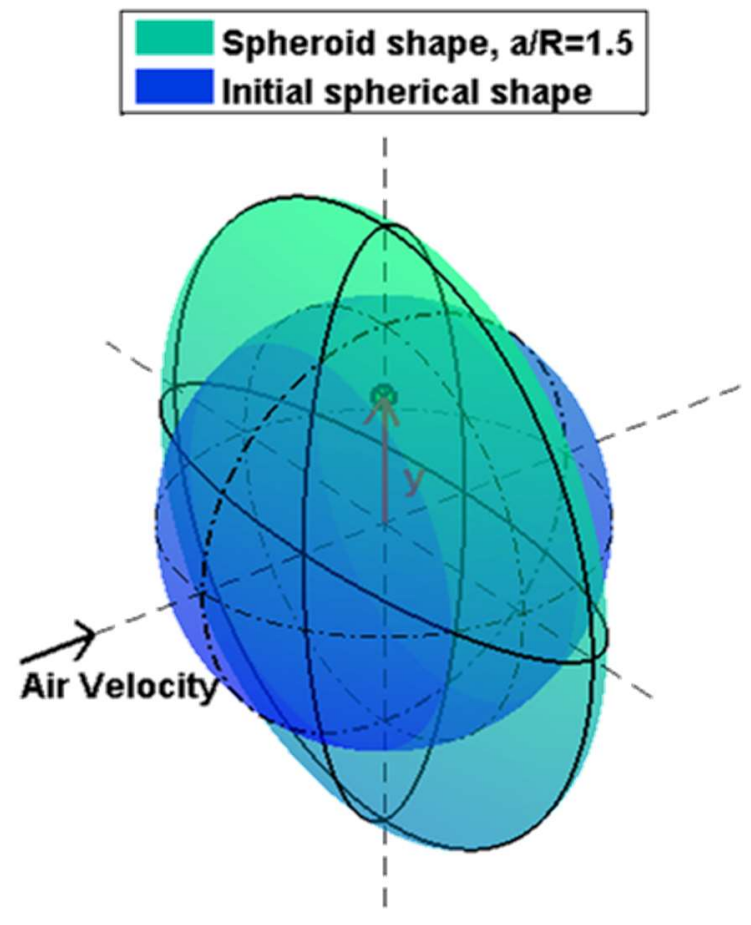

Figure A.1.3.1. DDB Model.

\section{A.1.4. Validity of the previous models.}

The previous models have been applied to droplets that are suddenly exposed to a constant air stream. Then, for droplet immersed in a continuously accelerated flow, these models should be applied with the actual instantaneous velocity between the droplet and the air (the slip velocity $V_{s}$ ), so the following change of independent variable is performed to obtain the dimensionless equation for each model.

$$
\begin{aligned}
t^{\prime} & =\frac{\left(t-t_{0}\right) V_{s}(t)}{R_{d}} \\
\frac{d t^{\prime}}{d t} & =\left(\frac{V_{s}}{R_{d}}\right)\left(1+B_{v} t^{\prime}\right) \\
\frac{d^{2} t^{\prime}}{d t^{2}} & =\left(\frac{V_{s}^{2}}{R_{d}^{2}}\right)\left(2 B_{v}+t^{\prime} B_{a}\right)
\end{aligned}
$$

where $B_{v}$ and $B_{a}$ are two new dimensionless numbers that appear naturally from the previous change of variable.

$$
B_{v}=\frac{R_{d}}{V_{s}^{2}} \frac{d V_{s}}{d t}
$$




$$
B_{a}=\frac{R_{d}{ }^{2}}{V_{s}^{3}} \frac{d^{2} V_{s}}{d t^{2}}
$$

$B_{v}$ could be expressed as a function of Bond number $B o=\left(\rho_{d} R_{d}{ }^{2} / \sigma\right) \cdot d V_{s} / d t$ and the others dimensionless parameters as follows

$$
B_{v}=\frac{B o}{K W e}
$$

Note that all the dimensionless parameters $\left(B o, W e, R e, B_{a}, B_{v}\right)$ are now time-dependent and depends on the slip velocity $V_{s}$ instead of $U_{\infty}$.

By introducing this new change of variable instead of the assumption of a constant slip velocity the Clark model yields the following equation

$$
\begin{aligned}
(K+1)\left[\left(1+B_{v} t^{\prime}\right)^{2}\right] \frac{d^{2} y^{\prime}}{d t^{\prime 2}}+\left[(K+1)\left(2 B_{v}+B_{a} t^{\prime}\right)\right. & \\
& \left.+\frac{9 \pi^{2}(N+1)\left(1+B_{v} t^{\prime}\right)}{4 R e}\right] \frac{d y^{\prime}}{d t^{\prime}}+\frac{9 \pi^{2}}{4 W e}\left(y^{\prime}-\frac{4}{3 \pi}\right)=\frac{2}{\pi}
\end{aligned}
$$

whereas the new DDB model equation is

$$
\begin{gathered}
K\left(1+B_{v} t^{\prime}\right)^{2} \frac{d^{2} y^{\prime}}{d t^{\prime 2}}+\left[K\left(2 B_{v}+B_{a} t^{\prime}\right)+\frac{4 N}{R e} \frac{\left(1+B_{v} t^{\prime}\right)}{y^{\prime 2}}\right] \frac{d y^{\prime}}{d t^{\prime}} \\
+\frac{3 c^{2}}{W e}\left(1-2\left(c y^{\prime}\right)^{-6}\right)=\frac{3}{8}
\end{gathered}
$$

and the new $\mathrm{TAB}$ equation can be expressed as

$$
K\left(1+B_{v} t^{\prime}\right)^{2} \frac{d^{2} y^{\prime}}{d t^{\prime 2}}+\left[K\left(2 B_{v}+B_{a} t^{\prime}\right)+\frac{5 N}{R e}\left(1+B_{v} t^{\prime}\right)\right] \frac{d y^{\prime}}{d t^{\prime}}+\frac{8}{W e}=\frac{2}{3}
$$

The solutions of the equations for TAB, Clark and DDB models are compared in figure A.1.4.1 with a experimental data obtained for a droplet of radius $0.524 \mathrm{~mm}$ immersed in the flow field generated by an airfoil model of chord $0.690 \mathrm{~mm}$ approaching at $90 \mathrm{~m} / \mathrm{s}$. 


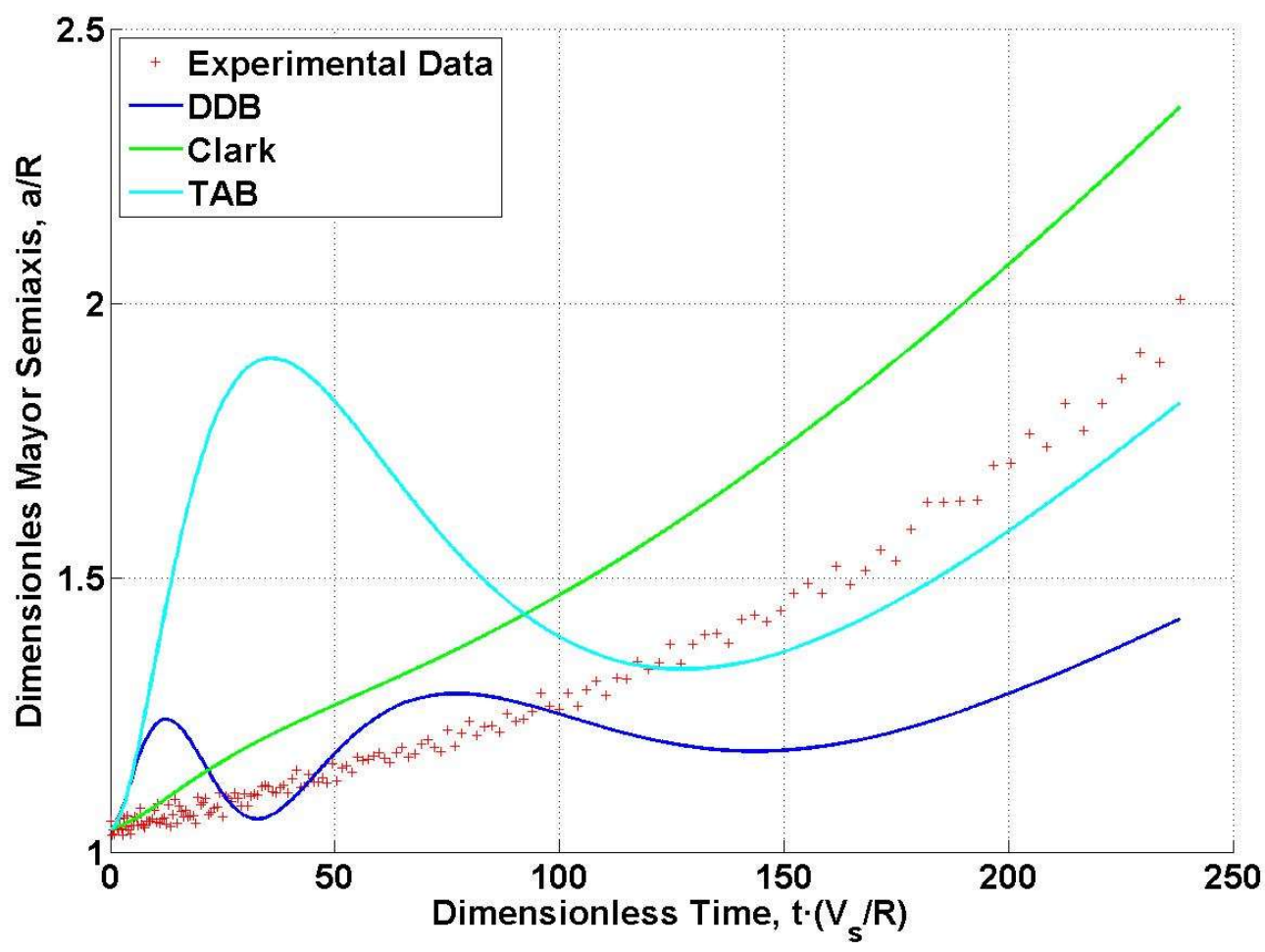

Figure A.1.4.1. Comparisons of previous models with experimental data. Case 110. Run Name 072011.14C.1.1. Airfoil Velocity: 90m/s. Chord Size: 690mm. Droplet Radius: $524 \mu \mathrm{m}$

Two years ago, Vargas (2012) applied the DDB model to the deformation of droplets assuming that at each location along the trajectory the droplets were in quasi-steady equilibrium. Experimental measured values of the slip velocity were used as part of the input to the model. The model was run at each location where experimental information was available up to breakup onset. He concluded that DDB model can be used in the analysis of the deformation of small and medium droplets, but for larger droplets (above $500 \mu \mathrm{m}$ of diameter) the model over-predicts the displacement of the center of mass by a large margin.

Due to the lack of accuracy of existing models when comparing with experimental data on approaching water droplets, it is apparent the necessity of improving these models. Therefore, a new deformation equation is now proposed.

\section{A.2. NEW DEFORMATION EQUATION.}

This new approach is again based on the analogy between an oscillation and distorting droplet, and a spring-mass system. It is assumed that the liquid droplet is deformed due to a pure extensional flow from an initial spherical shape of radius $R$ to an oblate spheroid. The problem is formulated in terms of the motion of the half-droplet center of mass. 
Forces are supposed to act through the center of mass, which is considered to be the center of mass of a half oblate spheroid of half-major axis $a$ and half-minor axis $b$. Being $y$ the center of mass and $c=8 / 3$, the relation between half-axes and the center of mass is known.

$$
a=c y=\frac{8}{3} y
$$

In the evolution from spherical droplet to oblate spheroid, the volume remains constant leading to the following relation between the spherical droplet radius $R$ and spheroid halfaxes.

$$
R_{d}{ }^{3}=a^{2} b
$$

The sum of all forces acting through the center of mass of the half-droplet is equal to the product of half-droplet mass $m$ and its acceleration $a_{c}$. Forces under consideration are viscosity forces, interfacial tension force and forces due to pressure flow field neglecting other forces.

$$
m_{d} \cdot a_{c}=F_{v}+F_{s t}+F_{P}
$$

\section{A.2.1. Viscous Force.}

The energy dissipation rate per unit volume in an incompressible flow is given by

$$
\phi=2 \mu_{d} e_{i j} e_{i j}
$$

where

$$
e_{i j}=\frac{1}{2}\left(\frac{\partial u_{i}}{\partial x_{j}}+\frac{\partial u_{j}}{\partial x_{i}}\right)
$$

The assumption of two-dimensional pure extensional flow,

$$
\frac{\partial u_{i}}{\partial x_{j}}=0 \forall i \neq j ; \frac{\partial u_{2}}{\partial x_{2}}=-\frac{\partial u_{1}}{\partial x_{1}}
$$

leads to an energy dissipation rate

$$
\Phi=4 \mu_{d}\left(\frac{\partial u_{1}}{\partial x_{1}}\right)^{2} \frac{2}{3} \pi R_{d}^{3}=\frac{8}{3} \mu_{d} \pi R_{d}{ }^{3} \frac{1}{y^{2}}\left(\frac{d y}{d t}\right)^{2}
$$


where

$$
u_{1}=\frac{d y}{d t}=\frac{\partial u_{1}}{\partial x_{1}} y \Rightarrow \frac{\partial u_{1}}{\partial x_{1}}=\frac{1}{y} \frac{d y}{d t}
$$

Finally, the viscous force is recovered by dividing the energy due to viscous dissipation into $d y$,

$$
F_{v}=-\frac{d E_{v i s \cos a}}{d y}=-\frac{\Phi d t}{d y}=-\frac{8}{3} \mu_{d} \pi R_{d}{ }^{3} \frac{1}{y^{2}}\left(\frac{d y}{d t}\right)
$$

\section{A.2.2. Interfacial Tension Force.}

The work performed to create an incremental increase of surface area is

$$
d W=\sigma d A_{s} .
$$

In the mechanical model, the force acting through the center of mass of the half drop is

$$
F_{s t}=-\frac{1}{2} \sigma \frac{d A_{S}}{d y}
$$

where $A_{S}$ is the droplet surface area. For an oblate spheroid, the surface area is given by

$$
\frac{A_{s}}{\pi R_{d}{ }^{2}}=2\left(\frac{a}{R_{d}}\right)^{2}+\frac{1}{\varepsilon}\left(\frac{b}{R_{d}}\right)^{2} \ln \left(\frac{1+\varepsilon}{1-\varepsilon}\right)
$$

where

$$
\varepsilon=\sqrt{1-\left(\frac{b}{a}\right)^{2}}
$$

Deriving now the droplet area surface

$$
\begin{aligned}
\frac{d\left(A_{s} / \pi R_{d}^{2}\right)}{d\left(a / R_{d}\right)} & =4\left(\frac{a}{R_{d}}\right)-\frac{4}{\left(a / R_{d}\right)^{5} \varepsilon} \ln \left(\frac{1+\varepsilon}{1-\varepsilon}\right) \\
& +\frac{3}{\left(a / R_{d}\right)^{11} \varepsilon}\left[\frac{2}{\varepsilon\left(1-\varepsilon^{2}\right)}-\frac{1}{\varepsilon} \ln \left(\frac{1+\varepsilon}{1-\varepsilon}\right)\right] .
\end{aligned}
$$

and using equation (3.50), we obtain 


$$
F_{s t}=-\frac{1}{2} \sigma \frac{d A_{s}}{d y}=-\frac{1}{2} \sigma c \frac{d A_{s}}{d a}
$$

In the following figure A.2.2.1 we can observe that the simplification $A_{s}=2 \pi\left(a^{2}+b^{2}\right)$ used in Ibrahim et al (1993) to obtain $A_{s}$ cannot be used in our case due to the deviation from zero for $d A_{s} / d a$ when the droplet is a sphere.

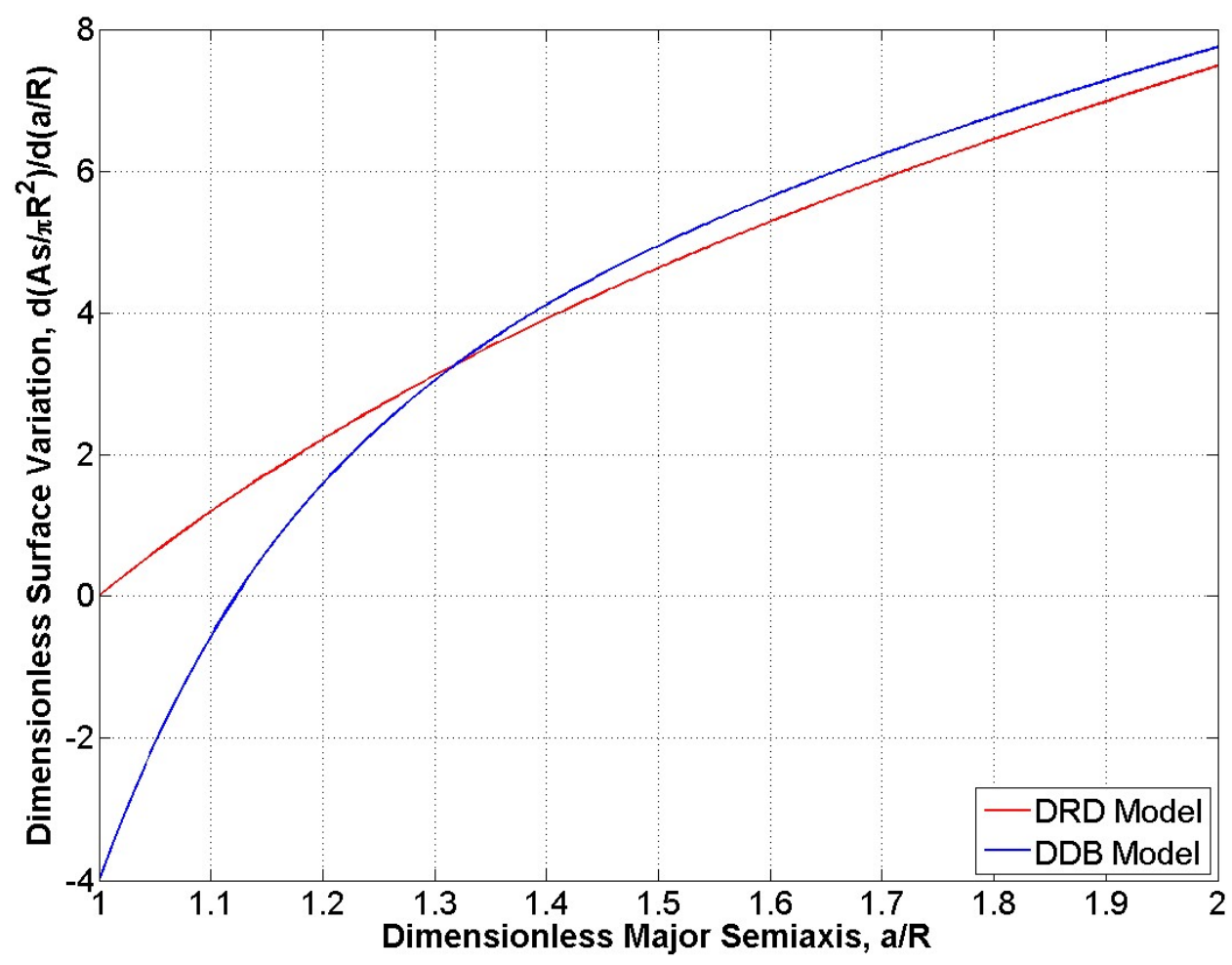

Figure A.2.2.1. Oblate spheroid surface area variation versus droplet deformation.

If the droplet was spherical, there should be a minimum in its surface area and accordingly the tension surface force should be zero, precluding any initial oscillation when there are no external forces. Note that in the DDB solution of figure A.1.4.1, oscillations appear at the beginning due to the surface area simplification.

\section{A.2.3. Pressure Force.}

We assume that the resultant of the pressure forces acting through the center of mass of half droplet is proportional to the dynamic pressure at the stagnation line and its projected area, being $c_{p}$ the proportionality coefficient. A first approach of the projected area is that of the equivalent spherical droplet

$$
F_{P}=c_{p} \frac{1}{2} \rho_{a} V_{s}^{2} \pi R_{d}^{2}
$$


The proportionality coefficient $c_{p}$ is to be estimated from experimental data.

\section{A.2.4. Equation for Droplet Ratio Deformation (DRD).}

By substitution of the mass of half droplet $m_{d}=\frac{2}{3} \pi R_{d}^{3} \rho_{d}$, its acceleration $a_{c}=\frac{d^{2} y}{d t^{2}}$ and all the forces proposed in equation (3.43), the equation governing droplet deformation is

$$
\frac{2}{3} \pi R_{d}{ }^{3} \rho_{d} \frac{d^{2} y}{d t^{2}}=-\frac{8}{3} \mu_{d} \pi R_{d}{ }^{3} \frac{1}{y^{2}}\left(\frac{d y}{d t}\right)-\frac{1}{2} \sigma c \frac{d A_{s}}{d a}+\frac{1}{2} c_{p} \rho_{a} V_{s}^{2} \pi R_{d}^{2}
$$

Defining the dimensionless quantities

$$
\mathrm{y}^{\prime}=\frac{\mathrm{y}}{R_{d}} ; \mathrm{a}^{\prime}=\frac{\mathrm{a}}{R_{d}} ; \mathrm{b}^{\prime}=\frac{\mathrm{b}}{R_{d}} ; \mathrm{A}_{\mathrm{s}}^{\prime}=\frac{\mathrm{A}_{\mathrm{s}}}{\pi R_{d}^{2}}
$$

and the dimensionless numbers, namely, densities ratios, viscosities ratios, Reynolds number and Weber number

$$
K=\frac{\rho_{d}}{\rho_{a}} ; N=\frac{\mu_{\mathrm{d}}}{\mu_{\mathrm{a}}} ; R e=\frac{\rho_{a} V_{s} R_{d}}{\mu_{a}} ; W e=\frac{\rho_{a} V_{s}^{2} R_{d}}{\sigma}
$$

Equation (3.56) can be rewritten as follows by dividing into $\frac{2}{3} \pi R_{d}^{2} \rho_{a} V_{s}^{2}$.

$$
K \frac{R_{d}^{2}}{V_{s}^{2}} \frac{d^{2} y^{\prime}}{d t^{2}}+\frac{4 N}{R e} \frac{1}{y^{2}} \frac{R_{d}}{V_{s}} \frac{d y^{\prime}}{d t}+\frac{3 c}{4 W e} \frac{d A_{s}^{\prime}}{d a^{\prime}}=\frac{3}{4} c_{p}
$$

Note that the slip velocity between the droplet and the air $V_{s}$ is not constant during the droplet deformation but depends on time. Weber and Reynolds number are instantaneous values. Assuming the slip velocity known as a function of time and changing the independent variable as in equations (3.60)-(3.62).

$$
\begin{gathered}
t^{\prime}=\frac{\left(t-t_{0}\right) V_{s}(t)}{R_{d}} \\
\frac{d t^{\prime}}{d t}=\left(\frac{V_{s}}{R_{d}}\right)\left(1+B_{v} t^{\prime}\right) \\
\frac{d^{2} t^{\prime}}{d t^{2}}=\left(\frac{V_{s}^{2}}{R_{d}^{2}}\right)\left(2 B_{v}+t^{\prime} B_{a}\right)
\end{gathered}
$$

where the dimensionless numbers $B_{v}$ and $B_{a}$ defined in equations (3.35) and (3.36) appear, equation. (3.59) can be rewritten as 


$$
\begin{aligned}
K\left(1+B_{v} t^{\prime}\right)^{2} & \frac{d^{2} y^{\prime}}{d t^{\prime 2}}+\left[K\left(2 B_{v}+B_{a} t^{\prime}\right)+\frac{4 N}{R e} \frac{1}{y^{2}}\left(1+B_{v} t^{\prime}\right)\right] \frac{d y^{\prime}}{d t^{\prime}}+\frac{3 c}{4 W e} \frac{d A_{s}^{\prime}}{d a^{\prime}} \\
& =\frac{3}{4} c_{p}
\end{aligned}
$$

where

$$
\frac{d A_{s}^{\prime}}{d a^{\prime}}=4\left(a^{\prime}\right)-\frac{4}{\left(a^{\prime}\right)^{5} \varepsilon} \ln \left(\frac{1+\varepsilon}{1-\varepsilon}\right)+\frac{3}{\left(a^{\prime}\right)^{11} \varepsilon}\left[\frac{2}{\varepsilon\left(1-\varepsilon^{2}\right)}-\frac{1}{\varepsilon} \ln \left(\frac{1+\varepsilon}{1-\varepsilon}\right)\right]
$$

Finally, note that $B_{v}$ could be expressed as a function of the instantaneous Bond number $B o$ and the former dimensionless parameters.

$$
B_{v}=\frac{B o}{K W e} \text { where } B o=\frac{\rho_{d} R_{d}^{2}}{\sigma} \frac{d V_{s}}{d t}
$$

\section{A.2.5. Pressure Proportionality Coefficient Estimate.}

The pressure proportionality coefficient $c_{p}$ used in equation (3.55) to evaluate pressure forces is estimated from experimental data. For this purpose, a total number of 159 experimental cases were analyzed, including different model sizes and velocities. For each case, the difference between the numerical solution obtained by the equation and experimental data, taken as an error, was depicted versus the value of $c_{p}$ used to obtain the numerical solution. In order to obtain a global $c_{p}$, an averaged curve is made out from all the cases analyzed and plotted in figure. A.2.5.1. There is a value of $c_{p}=0.93$ that minimizes the error, without any noticeable difference with respect to the particular flow field generated by each model and velocity. Then, we have chosen $c_{p}$ as a constant value equal to 0.93 . This value has been obtained by minimizing the error for different model and velocities, which leads to different accelerating flow fields. Therefore, the value encountered for the $c_{p}$ is of general applicability. Errors are calculated by the following expression for each experimental case and for each $c_{p}$.

$$
\text { Error }=\sqrt{\frac{1}{n} \sum\left(\frac{y_{i}{ }^{\prime}-y_{i}{ }^{\prime} \text { exp }}{y_{i}{ }^{\prime}{ }_{\text {exp }}}\right)^{2}}
$$




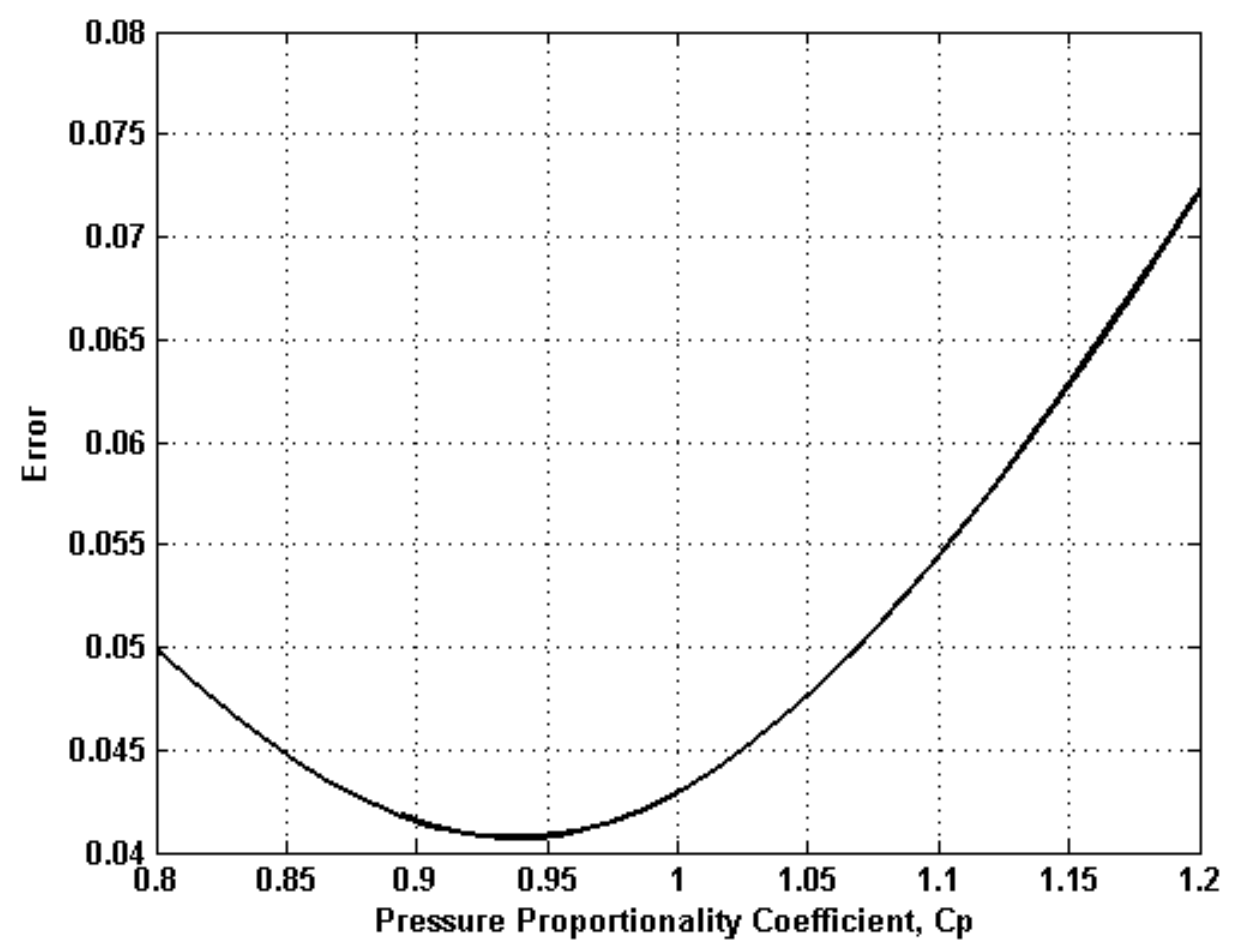

Figure. A.2.5.1. Mean Error of 159 cases vs. $\boldsymbol{c}_{\boldsymbol{p}}$

\section{A.3. EXPERIMENTAL VALIDATION.}

A total of 159 cases including three chord sizes $(199 \mathrm{~mm}, 470 \mathrm{~mm}$ and $690 \mathrm{~mm})$, five model velocities $(50 \mathrm{~m} / \mathrm{s}, 60 \mathrm{~m} / \mathrm{s}, 70 \mathrm{~m} / \mathrm{s}, 80 \mathrm{~m} / \mathrm{s}$ and $90 \mathrm{~m} / \mathrm{s})$ and droplet diameters between $0.3 \mathrm{~mm}$ and $1.4 \mathrm{~mm}$ have been used to validate the model. The equations of DRD, Clark and DDB models were solved using a fourth order Runge-Kutta and initial conditions obtained from experimental data. The initial conditions were obtained by the fourth order polynomial approximation of the maximum droplet half-diameter evolution of experimental data. Good results for the droplet deformation ratio equation solution were observed. Considering $c_{p}$ as 0.93 , the errors for the 159 cases analyzed were calculated as defined in equation (3.66) and showed in figure A.3.1. The mean error for a $c_{p}$ of 0.93 is 0.038 with a standard deviation of 0.0152 . 
Chord Size: $690 \mathrm{~mm}(0), 470 \mathrm{~mm}\left({ }^{*}\right)$ and $200 \mathrm{~mm}(+)$

Model Velocity (m/s): 90(black), 80(magenta), 70(green), 60(blue) and 50(red)

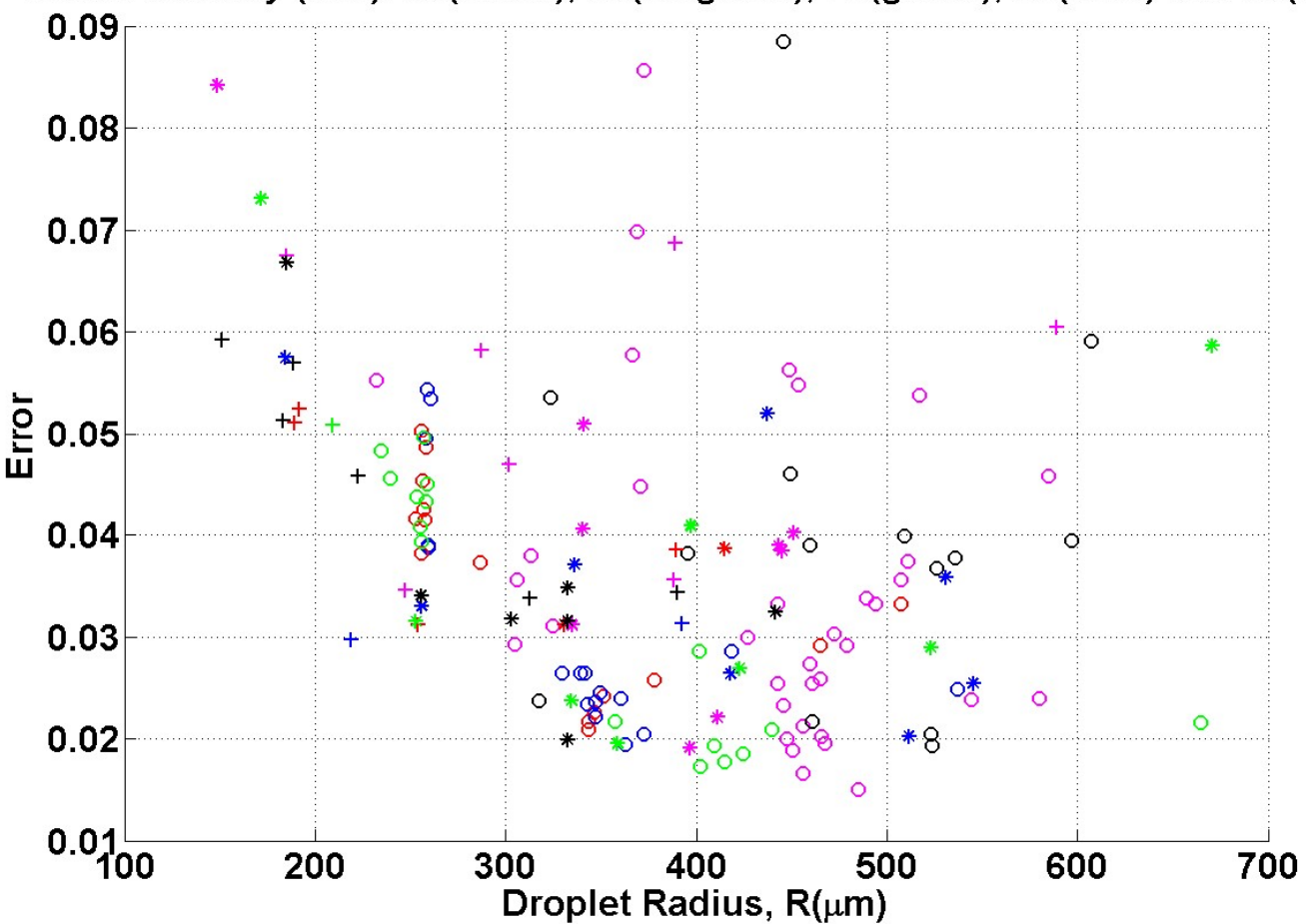

Figure A.3.1. Error vs. droplet radius for all the cases analyzed considering $c_{p}=0.93$. Each case is depicted by one point, and the color and the shape of the point indicates the model velocity, and the airfoil model chord size.

The influence of the droplet size in the results is studied. Results of five meaningful cases for different droplet sizes are shown and discussed in here (See figures A.3.2-A.3.6). These significant cases are summarized in table A.3.1. The biggest model and the maximum velocity model have been chosen due to the bigger deformation obtained in these cases, which allows to visualize better how the model works as compared to experimental data and previous models.

\begin{tabular}{ccccc}
\hline Case Number & Run Name & $\begin{array}{c}\text { Airfoil Velocity } \\
\text { Um }(\mathrm{m} / \mathrm{s})\end{array}$ & $\begin{array}{c}\text { Radius } \\
(\mu \mathrm{m})\end{array}$ & $\begin{array}{c}\text { Chord Size } \\
(\mathrm{mm})\end{array}$ \\
\hline 1249 & 072611.22 .4 .1 & 90 & 181 & 690 \\
1166 & $072611.20 \mathrm{~B} .1 .2$ & 90 & 317 & 690 \\
1172 & $072611.20 \mathrm{D} .1 .1$ & 90 & 421 & 690 \\
110 & $072011.14 \mathrm{C} .1 .1$ & 90 & 524 & 690 \\
1041 & 072611.16 .6 .2 & 90 & 565 & 690 \\
\hline
\end{tabular}

Table A.3.1. Data for droplets used to test the DRD, DDB and Clark Models.

For smaller droplets the experimental data show a higher level of dispersion because there are fewer pixels per droplet. For the droplet of case 1249 of radius of $181 \mu \mathrm{m}$, its diameter 
corresponds only to 5.2 pixels. Additionally, the total deformation for this droplet is also smaller.

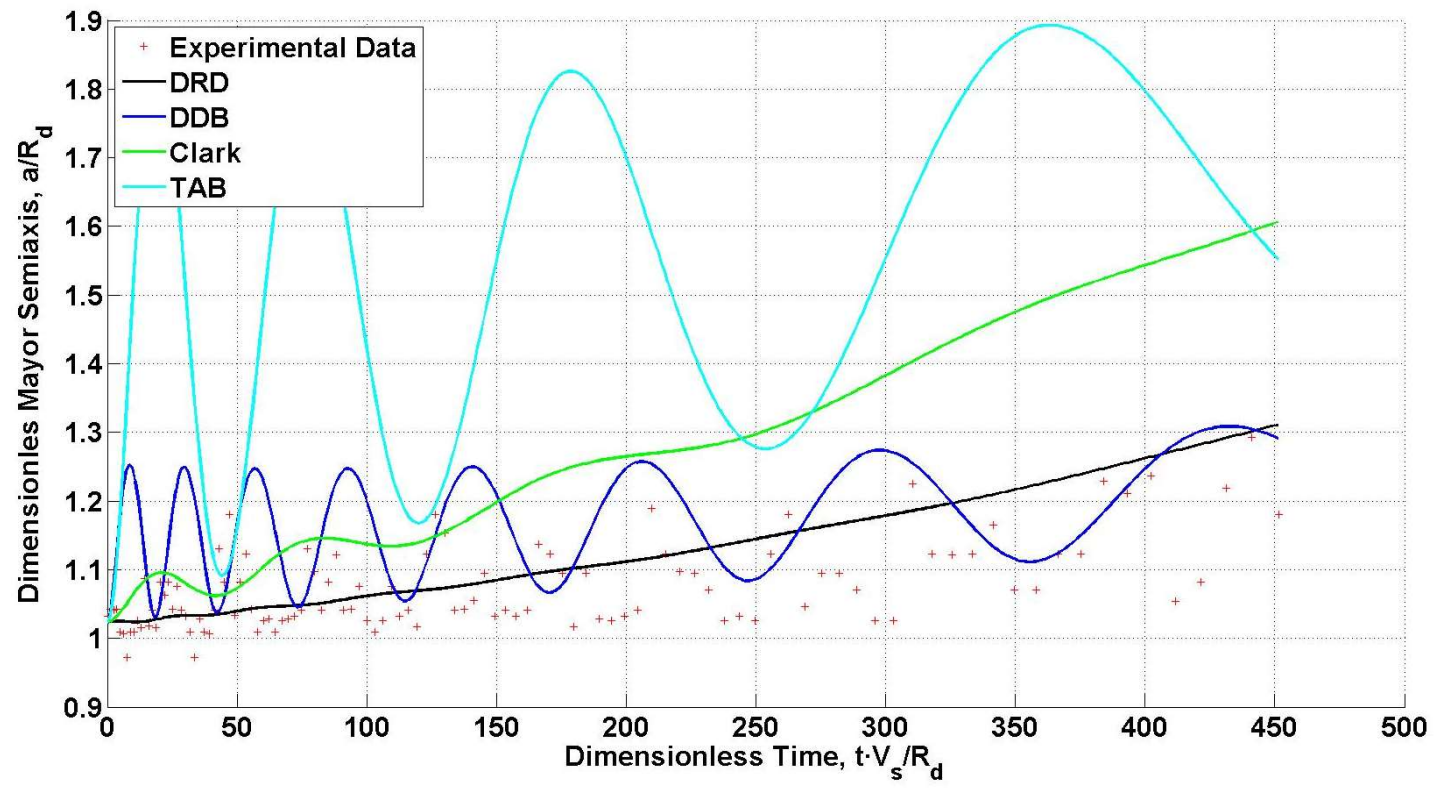

Figure A.3.2. Case 1249. Airfoil velocity: $90 \mathrm{~m} / \mathrm{s}$. Chord Size: $690 \mathrm{~mm}$. Droplet radius: $181 \mu \mathrm{m}$.

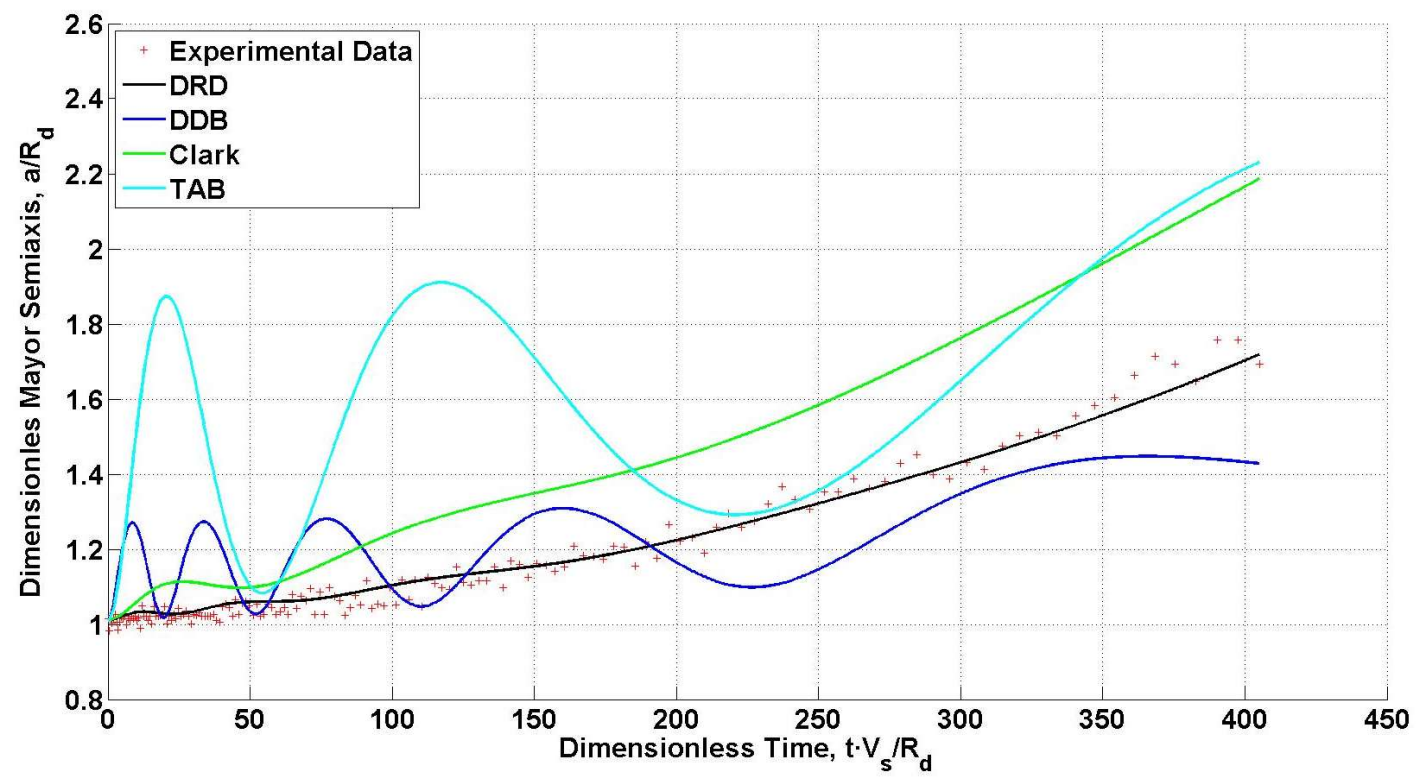

Figure A.3.3. Case 1166. Airfoil velocity: 90m/s. Chord Size: $690 \mathrm{~mm}$. Droplet radius: $317 \mu \mathrm{m}$. 


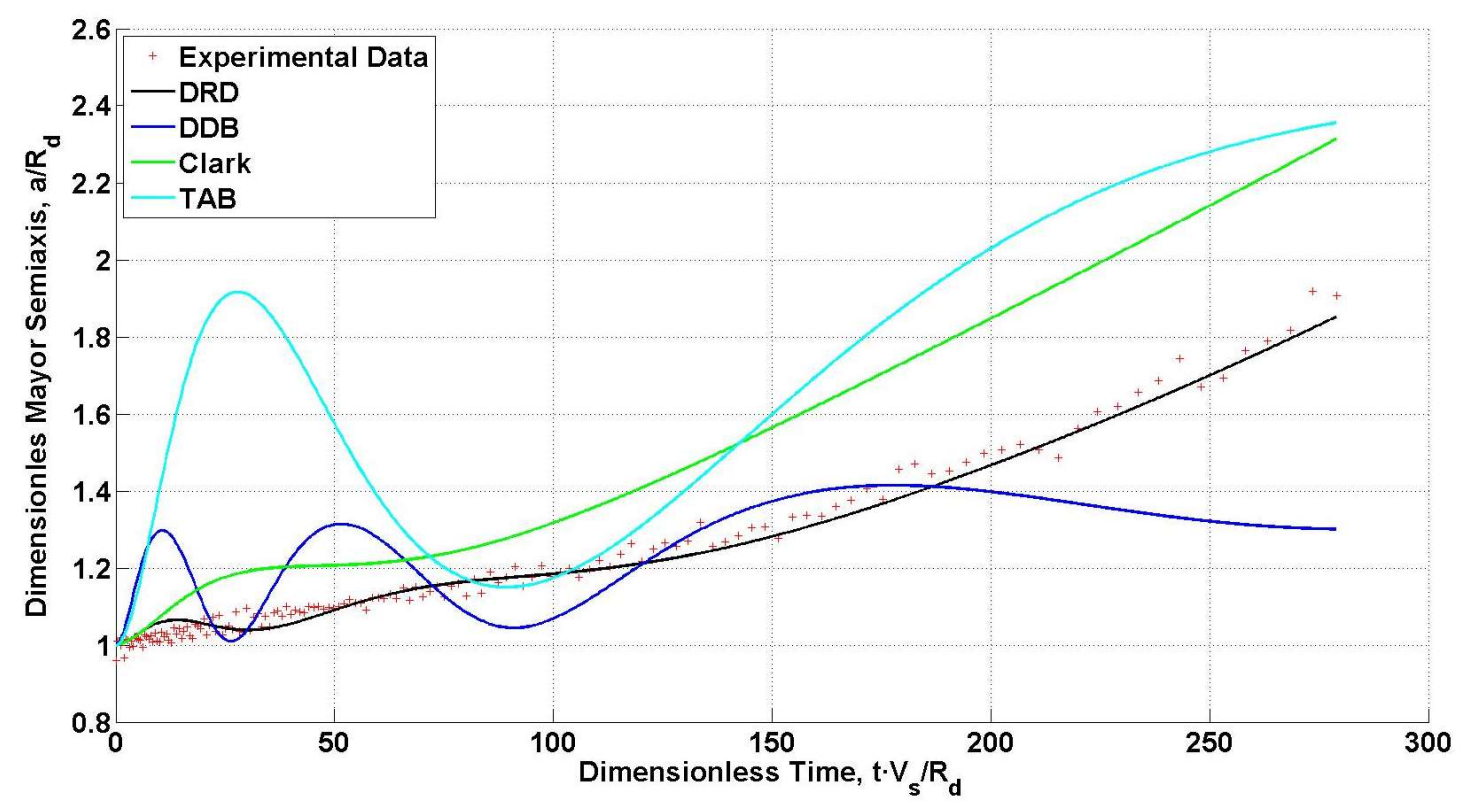

Figure A.3.4. Case 1172. Airfoil velocity: 90m/s. Chord Size: $690 \mathrm{~mm}$. Droplet radius: $421 \mu \mathrm{m}$.

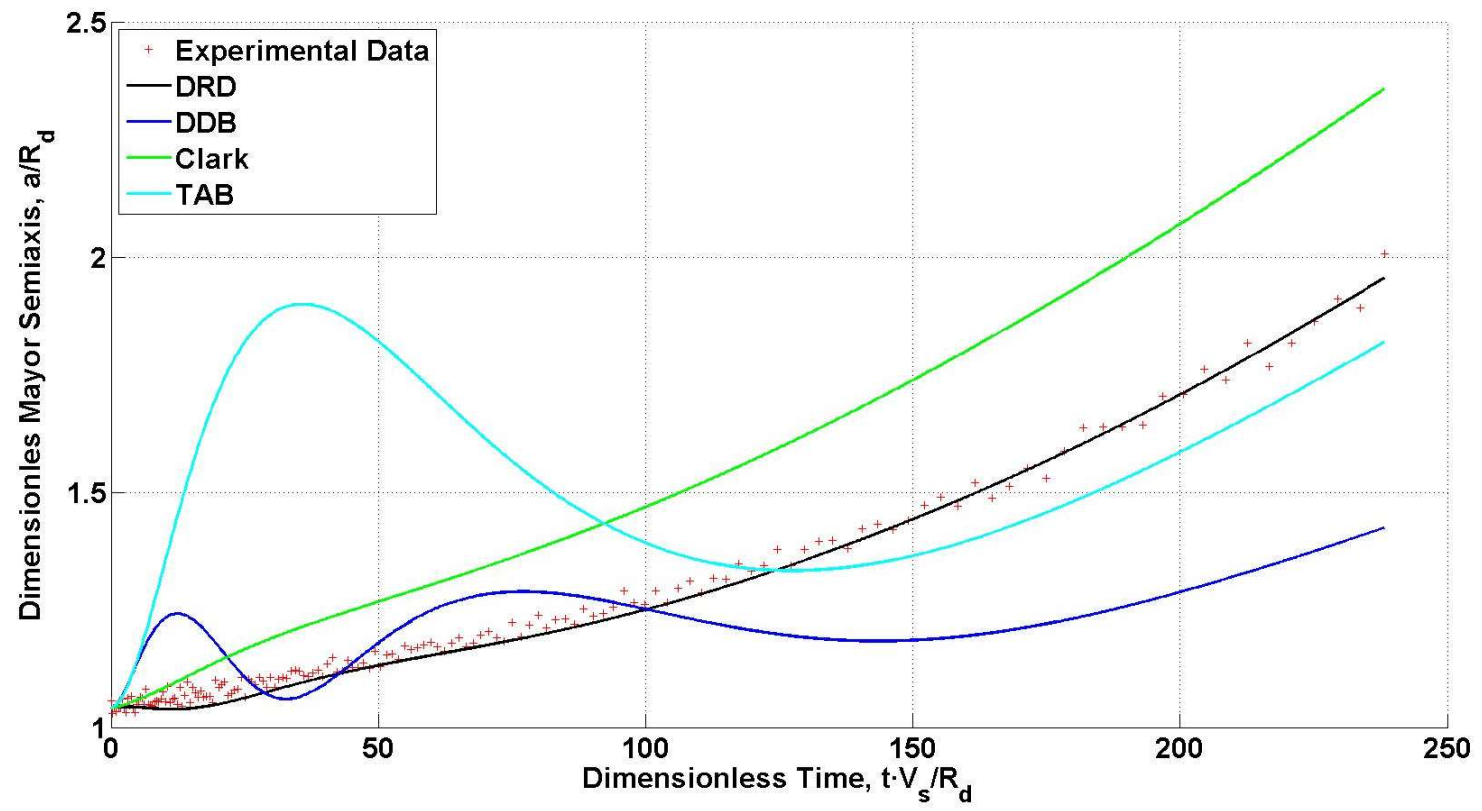

Figure A.3.5. Case 110. Airfoil velocity: $90 \mathrm{~m} / \mathrm{s}$. Chord Size: $690 \mathrm{~mm}$. Droplet radius: $524 \mu \mathrm{m}$. 


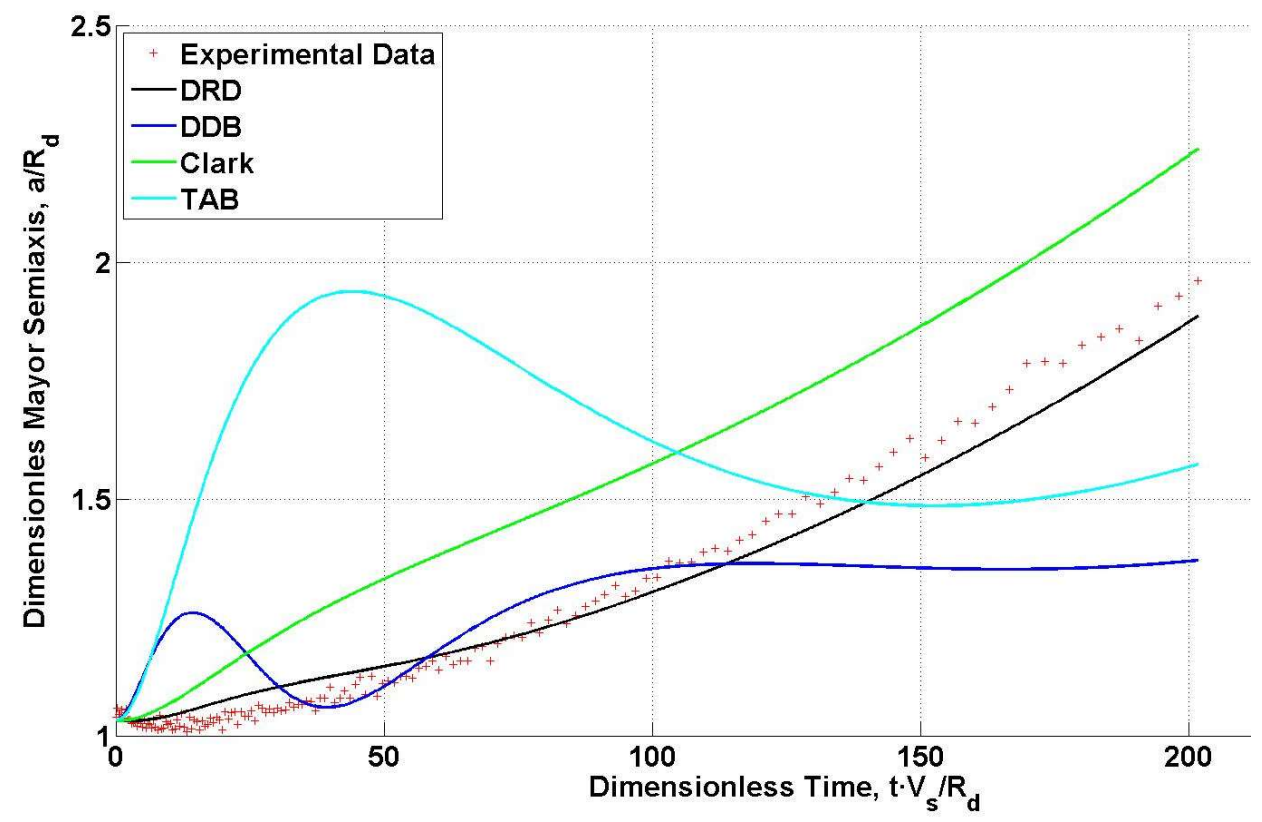

Figure A.3.6. Case 1041. Airfoil velocity: 90m/s. Chord Size: $690 \mathrm{~mm}$. Droplet radius: $565 \mu \mathrm{m}$.

The sequence of deformation for each case are shown in figures A.3.7 to A.3.11, and the evolution of the ratio deformation and the slip velocity, and the main dimensionless instantaneous number are shown in the tables A.3.1 to A.3.5

\begin{tabular}{ccccccccc}
\hline $\begin{array}{c}\text { Frame } \\
\#\end{array}$ & $\begin{array}{c}\text { Time } \\
(\mathrm{s})\end{array}$ & $\mathrm{a} / \mathrm{R}$ & $\begin{array}{c}\text { Slip } \\
\text { Velocity } \\
(\mathrm{m} / \mathrm{s})\end{array}$ & $\begin{array}{c}\text { Dimensionless } \\
\text { Time }\end{array}$ & Re & We & Bv & Ba \\
\hline 3143 & $5.33 \mathrm{E}-05$ & 1.009 & 16.76 & 4.93 & 203 & 0.84 & $7.14 \mathrm{E}-03$ & $7.83 \mathrm{E}-05$ \\
3163 & $3.20 \mathrm{E}-04$ & 1.028 & 20.16 & 35.56 & 244 & 1.22 & $6.54 \mathrm{E}-03$ & $6.44 \mathrm{E}-05$ \\
3173 & $4.53 \mathrm{E}-04$ & 1.043 & 22.27 & 55.63 & 269 & 1.48 & $6.22 \mathrm{E}-03$ & $5.72 \mathrm{E}-05$ \\
3183 & $5.87 \mathrm{E}-04$ & 1.097 & 24.71 & 79.91 & 299 & 1.83 & $5.88 \mathrm{E}-03$ & $5.01 \mathrm{E}-05$ \\
3193 & $7.20 \mathrm{E}-04$ & 1.076 & 27.57 & 109.40 & 333 & 2.27 & $5.53 \mathrm{E}-03$ & $4.32 \mathrm{E}-05$ \\
3203 & $8.53 \mathrm{E}-04$ & 1.095 & 30.91 & 145.40 & 374 & 2.86 & $5.16 \mathrm{E}-03$ & $3.66 \mathrm{E}-05$ \\
3208 & $9.20 \mathrm{E}-04$ & 1.137 & 32.80 & 166.30 & 397 & 3.22 & $4.97 \mathrm{E}-03$ & $3.36 \mathrm{E}-05$ \\
3213 & $9.87 \mathrm{E}-04$ & 1.028 & 34.84 & 189.46 & 421 & 3.63 & $4.78 \mathrm{E}-03$ & $3.06 \mathrm{E}-05$ \\
3218 & $1.05 \mathrm{E}-03$ & 1.123 & 37.06 & 215.18 & 448 & 4.11 & $4.58 \mathrm{E}-03$ & $2.78 \mathrm{E}-05$ \\
3223 & $1.12 \mathrm{E}-03$ & 1.032 & 39.47 & 243.68 & 477 & 4.66 & $4.39 \mathrm{E}-03$ & $2.52 \mathrm{E}-05$ \\
3228 & $1.19 \mathrm{E}-03$ & 1.095 & 42.10 & 275.38 & 509 & 5.30 & $4.19 \mathrm{E}-03$ & $2.27 \mathrm{E}-05$ \\
3233 & $1.25 \mathrm{E}-03$ & 1.225 & 44.96 & 310.56 & 544 & 6.04 & $4.00 \mathrm{E}-03$ & $2.04 \mathrm{E}-05$ \\
3238 & $1.32 \mathrm{E}-03$ & 1.070 & 48.05 & 349.59 & 581 & 6.90 & $3.81 \mathrm{E}-03$ & $1.83 \mathrm{E}-05$ \\
3243 & $1.39 \mathrm{E}-03$ & 1.211 & 51.43 & 393.09 & 622 & 7.91 & $3.62 \mathrm{E}-03$ & $1.63 \mathrm{E}-05$ \\
3248 & $1.45 \mathrm{E}-03$ & 1.292 & 55.08 & 441.24 & 666 & 9.07 & $3.44 \mathrm{E}-03$ & $1.45 \mathrm{E}-05$ \\
3249 & $1.47 \mathrm{E}-03$ & 1.181 & 55.86 & 451.57 & 676 & 9.33 & $3.40 \mathrm{E}-03$ & $1.42 \mathrm{E}-05$ \\
\hline
\end{tabular}

Table A.3.1. Case1249. Droplet Deformation Evolution. Airfoil velocity: 90m/s. 


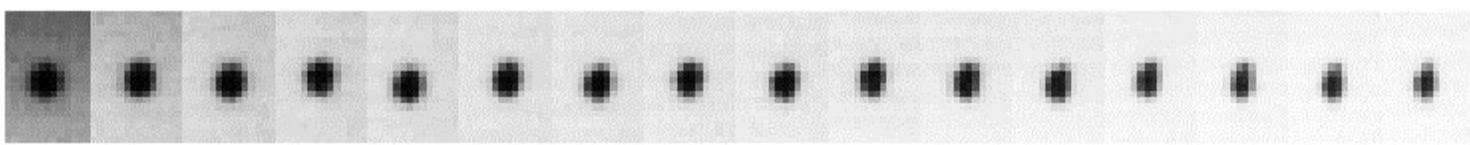

Figure A.3.7. Case1249. Droplet Deformation Evolution. Airfoil velocity: 90m/s. Chord Size: $690 \mathrm{~mm}$. Droplet radius: $181 \mu \mathrm{m}$.

\begin{tabular}{ccccccccc}
\hline $\begin{array}{c}\text { Frame } \\
\#\end{array}$ & $\begin{array}{c}\text { Time } \\
(\mathrm{s})\end{array}$ & $\mathrm{a} / \mathrm{R}$ & $\begin{array}{c}\text { Slip } \\
\text { Velocity } \\
(\mathrm{m} / \mathrm{s})\end{array}$ & $\begin{array}{c}\text { Dimensionless } \\
\text { Time }\end{array}$ & Re & We & Bv & Ba \\
\hline 79 & $5.33 \mathrm{E}-05$ & 1.014 & 12.03 & 2.02 & 254 & 0.76 & $1.52 \mathrm{E}-02$ & $1.19 \mathrm{E}-04$ \\
99 & $3.20 \mathrm{E}-04$ & 1.009 & 13.94 & 14.06 & 295 & 1.02 & $1.30 \mathrm{E}-02$ & $2.12 \mathrm{E}-04$ \\
119 & $5.87 \mathrm{E}-04$ & 1.027 & 16.34 & 30.23 & 346 & 1.40 & $1.19 \mathrm{E}-02$ & $2.28 \mathrm{E}-04$ \\
139 & $8.53 \mathrm{E}-04$ & 1.053 & 19.46 & 52.35 & 412 & 1.98 & $1.12 \mathrm{E}-02$ & $2.05 \mathrm{E}-04$ \\
149 & $9.87 \mathrm{E}-04$ & 1.043 & 21.38 & 66.49 & 452 & 2.39 & $1.08 \mathrm{E}-02$ & $1.86 \mathrm{E}-04$ \\
159 & $1.12 \mathrm{E}-03$ & 1.025 & 23.60 & 83.33 & 499 & 2.91 & $1.03 \mathrm{E}-02$ & $1.64 \mathrm{E}-04$ \\
169 & $1.25 \mathrm{E}-03$ & 1.119 & 26.19 & 103.50 & 554 & 3.59 & $9.83 \mathrm{E}-03$ & $1.42 \mathrm{E}-04$ \\
179 & $1.39 \mathrm{E}-03$ & 1.105 & 29.24 & 127.82 & 618 & 4.47 & $9.25 \mathrm{E}-03$ & $1.20 \mathrm{E}-04$ \\
189 & $1.52 \mathrm{E}-03$ & 1.142 & 32.83 & 157.31 & 694 & 5.64 & $8.61 \mathrm{E}-03$ & $9.94 \mathrm{E}-05$ \\
199 & $1.65 \mathrm{E}-03$ & 1.176 & 37.07 & 193.21 & 784 & 7.18 & $7.92 \mathrm{E}-03$ & $8.08 \mathrm{E}-05$ \\
209 & $1.79 \mathrm{E}-03$ & 1.367 & 42.08 & 237.04 & 890 & 9.26 & $7.20 \mathrm{E}-03$ & $6.45 \mathrm{E}-05$ \\
214 & $1.85 \mathrm{E}-03$ & 1.388 & 44.90 & 262.36 & 950 & 10.54 & $6.84 \mathrm{E}-03$ & $5.74 \mathrm{E}-05$ \\
219 & $1.92 \mathrm{E}-03$ & 1.399 & 47.96 & 290.27 & 1014 & 12.02 & $6.49 \mathrm{E}-03$ & $5.09 \mathrm{E}-05$ \\
224 & $1.99 \mathrm{E}-03$ & 1.502 & 51.22 & 320.79 & 1083 & 13.72 & $6.16 \mathrm{E}-03$ & $4.51 \mathrm{E}-05$ \\
229 & $2.05 \mathrm{E}-03$ & 1.603 & 54.72 & 354.23 & 1157 & 15.66 & $5.84 \mathrm{E}-03$ & $3.99 \mathrm{E}-05$ \\
232 & $2.09 \mathrm{E}-03$ & 1.692 & 56.89 & 375.47 & 1203 & 16.93 & $5.66 \mathrm{E}-03$ & $3.72 \mathrm{E}-05$ \\
\hline
\end{tabular}

Table A.3.2. Case1166. Droplet Deformation Evolution. Airfoil velocity: 90m/s. Chord Size: $690 \mathrm{~mm}$. Droplet radius: $317 \mu \mathrm{m}$.

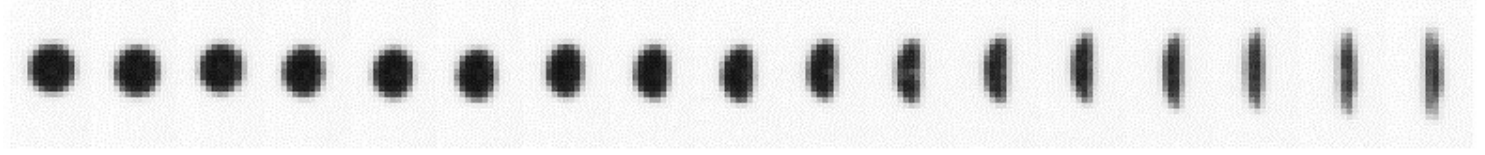

Figure A.3.8. Case1166. Droplet Deformation Evolution. Airfoil velocity: 90m/s. Chord Size: $690 \mathrm{~mm}$. Droplet radius: $317 \mu \mathrm{m}$.

\begin{tabular}{ccccccccc}
\hline $\begin{array}{c}\text { Frame } \\
\#\end{array}$ & $\begin{array}{c}\text { Time } \\
(\mathrm{s})\end{array}$ & $\mathrm{a} / \mathrm{R}$ & $\begin{array}{c}\text { Slip } \\
\text { Velocity } \\
(\mathrm{m} / \mathrm{s})\end{array}$ & $\begin{array}{c}\text { Dimensionless } \\
\text { Time }\end{array}$ & Re & We & $\mathrm{Bv}$ & $\mathrm{Ba}$ \\
\hline 93 & $5.33 \mathrm{E}-05$ & 1.010 & 11.82 & 1.50 & 332 & 0.97 & $2.20 \mathrm{E}-02$ & $2.49 \mathrm{E}-04$ \\
113 & $3.20 \mathrm{E}-04$ & 1.034 & 13.85 & 10.53 & 389 & 1.33 & $1.85 \mathrm{E}-02$ & $4.16 \mathrm{E}-04$ \\
133 & $5.87 \mathrm{E}-04$ & 1.035 & 16.40 & 22.86 & 460 & 1.87 & $1.67 \mathrm{E}-02$ & $4.29 \mathrm{E}-04$ \\
153 & $8.53 \mathrm{E}-04$ & 1.080 & 19.72 & 39.96 & 553 & 2.70 & $1.54 \mathrm{E}-02$ & $3.72 \mathrm{E}-04$ \\
163 & $9.87 \mathrm{E}-04$ & 1.100 & 21.76 & 50.99 & 611 & 3.28 & $1.47 \mathrm{E}-02$ & $3.32 \mathrm{E}-04$ \\
173 & $1.12 \mathrm{E}-03$ & 1.121 & 24.13 & 64.20 & 677 & 4.04 & $1.40 \mathrm{E}-02$ & $2.90 \mathrm{E}-04$ \\
183 & $1.25 \mathrm{E}-03$ & 1.129 & 26.90 & 80.09 & 755 & 5.02 & $1.32 \mathrm{E}-02$ & $2.47 \mathrm{E}-04$ \\
\hline
\end{tabular}




\begin{tabular}{lllllclll}
\hline 193 & $1.39 \mathrm{E}-03$ & 1.179 & 30.16 & 99.36 & 847 & 6.31 & $1.23 \mathrm{E}-02$ & $2.07 \mathrm{E}-04$ \\
203 & $1.52 \mathrm{E}-03$ & 1.250 & 34.01 & 122.81 & 955 & 8.03 & $1.14 \mathrm{E}-02$ & $1.69 \mathrm{E}-04$ \\
208 & $1.59 \mathrm{E}-03$ & 1.257 & 36.20 & 136.43 & 1016 & 9.09 & $1.09 \mathrm{E}-02$ & $1.52 \mathrm{E}-04$ \\
213 & $1.65 \mathrm{E}-03$ & 1.277 & 38.57 & 151.49 & 1083 & 10.32 & $1.04 \mathrm{E}-02$ & $1.36 \mathrm{E}-04$ \\
218 & $1.72 \mathrm{E}-03$ & 1.377 & 41.15 & 168.12 & 1155 & 11.75 & $9.89 \mathrm{E}-03$ & $1.21 \mathrm{E}-04$ \\
223 & $1.79 \mathrm{E}-03$ & 1.445 & 43.94 & 186.49 & 1233 & 13.40 & $9.39 \mathrm{E}-03$ & $1.08 \mathrm{E}-04$ \\
228 & $1.85 \mathrm{E}-03$ & 1.520 & 46.96 & 206.76 & 1318 & 15.30 & $8.90 \mathrm{E}-03$ & $9.53 \mathrm{E}-05$ \\
233 & $1.92 \mathrm{E}-03$ & 1.619 & 50.20 & 228.94 & 1409 & 17.48 & $8.44 \mathrm{E}-03$ & $8.44 \mathrm{E}-05$ \\
238 & $1.99 \mathrm{E}-03$ & 1.693 & 53.63 & 253.07 & 1505 & 19.96 & $8.00 \mathrm{E}-03$ & $7.48 \mathrm{E}-05$ \\
\hline
\end{tabular}

Table A.3.3. Case1172. Droplet Deformation Evolution. Airfoil velocity: 90m/s. Chord Size: $690 \mathrm{~mm}$. Droplet radius: $421 \mu \mathrm{m}$.

\section{$\bullet \bullet \bullet \bullet \bullet+11111111$}

Figure A.3.9. Case1 172. Droplet Deformation Evolution. Airfoil velocity: 90m/s. Chord Size: $690 \mathrm{~mm}$. Droplet radius: $421 \mu \mathrm{m}$.

\begin{tabular}{ccccccccc}
\hline $\begin{array}{c}\text { Frame } \\
\#\end{array}$ & $\begin{array}{c}\text { Time } \\
(\mathrm{s})\end{array}$ & $\mathrm{a} / \mathrm{R}$ & $\begin{array}{c}\text { Slip } \\
\text { Velocity } \\
(\mathrm{m} / \mathrm{s})\end{array}$ & $\begin{array}{c}\text { Dimensionless } \\
\text { Time }\end{array}$ & $\mathrm{Re}$ & $\mathrm{We}$ & $\mathrm{Bv}$ & $\mathrm{Ba}$ \\
\hline 61 & $5.33 \mathrm{E}-05$ & 1.048 & 11.59 & 1.18 & 405 & 1.16 & $2.38 \mathrm{E}-02$ & $4.53 \mathrm{E}-04$ \\
81 & $3.20 \mathrm{E}-04$ & 1.055 & 13.29 & 8.12 & 464 & 1.53 & $2.13 \mathrm{E}-02$ & $6.57 \mathrm{E}-04$ \\
101 & $5.87 \mathrm{E}-04$ & 1.064 & 15.47 & 17.33 & 540 & 2.07 & $2.00 \mathrm{E}-02$ & $6.83 \mathrm{E}-04$ \\
111 & $7.20 \mathrm{E}-04$ & 1.079 & 16.80 & 23.09 & 587 & 2.44 & $1.95 \mathrm{E}-02$ & $6.55 \mathrm{E}-04$ \\
121 & $8.53 \mathrm{E}-04$ & 1.106 & 18.32 & 29.84 & 640 & 2.90 & $1.90 \mathrm{E}-02$ & $6.09 \mathrm{E}-04$ \\
131 & $9.87 \mathrm{E}-04$ & 1.117 & 20.07 & 37.80 & 701 & 3.48 & $1.84 \mathrm{E}-02$ & $5.53 \mathrm{E}-04$ \\
141 & $1.12 \mathrm{E}-03$ & 1.136 & 22.10 & 47.25 & 772 & 4.22 & $1.77 \mathrm{E}-02$ & $4.90 \mathrm{E}-04$ \\
151 & $1.25 \mathrm{E}-03$ & 1.174 & 24.46 & 58.54 & 854 & 5.17 & $1.69 \mathrm{E}-02$ & $4.25 \mathrm{E}-04$ \\
161 & $1.39 \mathrm{E}-03$ & 1.191 & 27.23 & 72.09 & 951 & 6.40 & $1.60 \mathrm{E}-02$ & $3.62 \mathrm{E}-04$ \\
171 & $1.52 \mathrm{E}-03$ & 1.253 & 30.47 & 88.44 & 1064 & 8.02 & $1.49 \mathrm{E}-02$ & $3.02 \mathrm{E}-04$ \\
181 & $1.65 \mathrm{E}-03$ & 1.312 & 34.30 & 108.26 & 1198 & 10.16 & $1.38 \mathrm{E}-02$ & $2.47 \mathrm{E}-04$ \\
191 & $1.79 \mathrm{E}-03$ & 1.395 & 38.80 & 132.36 & 1355 & 13.00 & $1.26 \mathrm{E}-02$ & $1.99 \mathrm{E}-04$ \\
201 & $1.92 \mathrm{E}-03$ & 1.521 & 44.08 & 161.58 & 1539 & 16.78 & $1.14 \mathrm{E}-02$ & $1.58 \mathrm{E}-04$ \\
206 & $1.99 \mathrm{E}-03$ & 1.588 & 47.03 & 178.39 & 1642 & 19.10 & $1.08 \mathrm{E}-02$ & $1.40 \mathrm{E}-04$ \\
211 & $2.05 \mathrm{E}-03$ & 1.704 & 50.19 & 196.75 & 1753 & 21.75 & $1.03 \mathrm{E}-02$ & $1.25 \mathrm{E}-04$ \\
216 & $2.12 \mathrm{E}-03$ & 1.768 & 53.54 & 216.71 & 1870 & 24.75 & $9.75 \mathrm{E}-03$ & $1.11 \mathrm{E}-04$ \\
\hline
\end{tabular}

Table A.3.4. Case110. Droplet Deformation Evolution. Airfoil velocity: 90m/s. Chord Size: $690 \mathrm{~mm}$. Droplet radius: $524 \mu \mathrm{m}$. 


\section{$\bullet \bullet \bullet \bullet \bullet \bullet \bullet \bullet \bullet 1111111$}

Figure A.3.10. Case110. Droplet Deformation Evolution. Airfoil velocity: 90m/s. Chord Size: $690 \mathrm{~mm}$. Droplet radius: $524 \mu \mathrm{m}$.

\begin{tabular}{ccccccccc}
\hline $\begin{array}{c}\text { Frame } \\
\#\end{array}$ & $\begin{array}{c}\text { Time } \\
(\mathrm{s})\end{array}$ & $\mathrm{a} / \mathrm{R}$ & $\begin{array}{c}\text { Slip } \\
\text { Velocity } \\
(\mathrm{m} / \mathrm{s})\end{array}$ & $\begin{array}{c}\text { Dimensionless } \\
\text { Time }\end{array}$ & $\mathrm{Re}$ & $\mathrm{We}$ & $\mathrm{Bv}$ & $\mathrm{Ba}$ \\
\hline 2170 & $5.33 \mathrm{E}-05$ & 1.055 & 11.96 & 1.13 & 450 & 1.33 & $2.50 \mathrm{E}-02$ & $4.86 \mathrm{E}-04$ \\
2190 & $3.20 \mathrm{E}-04$ & 1.012 & 13.73 & 7.78 & 517 & 1.76 & $2.24 \mathrm{E}-02$ & $7.46 \mathrm{E}-04$ \\
2210 & $5.87 \mathrm{E}-04$ & 1.020 & 16.01 & 16.62 & 603 & 2.39 & $2.11 \mathrm{E}-02$ & $7.73 \mathrm{E}-04$ \\
2230 & $8.53 \mathrm{E}-04$ & 1.056 & 19.00 & 28.69 & 715 & 3.36 & $2.01 \mathrm{E}-02$ & $6.80 \mathrm{E}-04$ \\
2240 & $9.87 \mathrm{E}-04$ & 1.072 & 20.84 & 36.40 & 785 & 4.05 & $1.95 \mathrm{E}-02$ & $6.12 \mathrm{E}-04$ \\
2250 & $1.12 \mathrm{E}-03$ & 1.124 & 22.99 & 45.57 & 866 & 4.92 & $1.87 \mathrm{E}-02$ & $5.38 \mathrm{E}-04$ \\
2260 & $1.25 \mathrm{E}-03$ & 1.144 & 25.49 & 56.54 & 960 & 6.05 & $1.78 \mathrm{E}-02$ & $4.62 \mathrm{E}-04$ \\
2270 & $1.39 \mathrm{E}-03$ & 1.158 & 28.41 & 69.74 & 1070 & 7.52 & $1.67 \mathrm{E}-02$ & $3.89 \mathrm{E}-04$ \\
2280 & $1.52 \mathrm{E}-03$ & 1.255 & 31.86 & 85.71 & 1200 & 9.45 & $1.55 \mathrm{E}-02$ & $3.22 \mathrm{E}-04$ \\
2290 & $1.65 \mathrm{E}-03$ & 1.365 & 35.91 & 105.09 & 1352 & 12.01 & $1.43 \mathrm{E}-02$ & $2.61 \mathrm{E}-04$ \\
2295 & $1.72 \mathrm{E}-03$ & 1.412 & 38.19 & 116.29 & 1438 & 13.58 & $1.36 \mathrm{E}-02$ & $2.34 \mathrm{E}-04$ \\
2300 & $1.79 \mathrm{E}-03$ & 1.506 & 40.67 & 128.61 & 1532 & 15.40 & $1.30 \mathrm{E}-02$ & $2.08 \mathrm{E}-04$ \\
2305 & $1.85 \mathrm{E}-03$ & 1.568 & 43.33 & 142.15 & 1632 & 17.48 & $1.24 \mathrm{E}-02$ & $1.86 \mathrm{E}-04$ \\
2310 & $1.92 \mathrm{E}-03$ & 1.663 & 46.19 & 156.98 & 1740 & 19.87 & $1.18 \mathrm{E}-02$ & $1.65 \mathrm{E}-04$ \\
2315 & $1.99 \mathrm{E}-03$ & 1.791 & 49.24 & 173.16 & 1855 & 22.58 & $1.12 \mathrm{E}-02$ & $1.47 \mathrm{E}-04$ \\
2320 & $2.05 \mathrm{E}-03$ & 1.835 & 52.46 & 190.66 & 1976 & 25.62 & $1.06 \mathrm{E}-02$ & $1.30 \mathrm{E}-04$ \\
\hline
\end{tabular}

Table A.3.5. Case1041. Droplet Deformation Evolution. Airfoil velocity: 90m/s. Chord Size: $690 \mathrm{~mm}$. Droplet radius: $565 \mu \mathrm{m}$.

\section{$\bullet \bullet \bullet \bullet \bullet \bullet \bullet \bullet 11111111$}

Figure A.3.11. Case1041. Droplet Deformation Evolution. Airfoil velocity: 90m/s. Chord Size: $690 \mathrm{~mm}$. Droplet radius: $565 \mu \mathrm{m}$.

There are several observations that can be inferred from the cases presented in here. First of all, DRD's model seems to fit better the experimental data as compared to the other models. However, there appears an initial oscillation that could be due to the initial conditions error given by experimental data. If the initial conditions are slightly variated, these oscillations disappear. The Clark's model exhibits a similar behavior. The DDB solution presents a considerably greater initial oscillation. In this case, another source of error is introduced. As pointed in previous section describing the model, the surface droplet simplification, intrinsic to DDB model, does not work properly when the droplet is almost spherical (See figure A.3.12). 
As the slip velocity between the air and the droplet increases, the pressure force also increases leading to a more significant effect as droplet gets more distorted. In the DRD model the pressure force is estimated to be approximately the double of that of the DDB model, which makes the DRD solution to fit better than the DDB solution at the end of the curve. In Clark's model, although the pressure force is smaller than that of DRD, the deformation solution is higher at the end of the curve. This is due to the fact that the estimation for the surface tension force is also smaller than in DRD and DDB models but in a greater proportion. The surface tension force is proportional to the surface variation with the deformation, which can be observed in figure A.3.12 to be smaller for Clark's model.

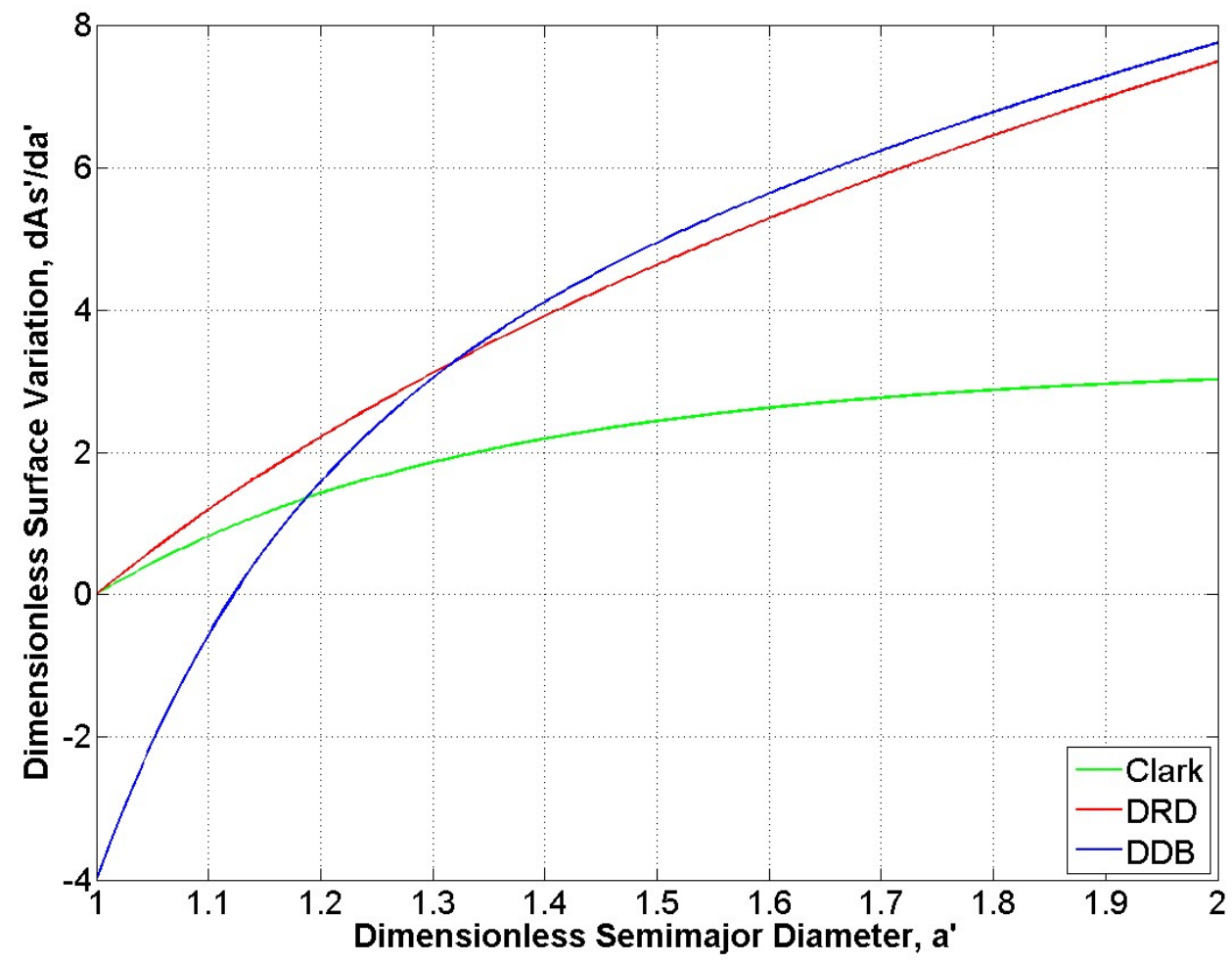

Figure A.3.12. Droplet surface variation with droplet deformation.

\section{A.4. CONCLUSIONS.}

A new equation has been proposed for the deformation of droplets based on a springmass analogy similar to Clark's and the DDB models. All three models include only forces due to surface tension, viscosity and pressure. A comparison has been made between the three models and experimental data of water droplets approaching the leading 
edge of an airfoil along the stagnation line. The following conclusions can be stated about the results obtained in this appendix:

1. The new equation predicts accurately the deformation of droplets of radius between $150 \mu \mathrm{m}$ and $700 \mu \mathrm{m}$ being distorted by an increasing exponential velocity flow field as experienced by a droplet approaching the leading edge of an airfoil.

2. The surface tension force model proposed, based on an oblate spheroid deformation, works well without the simplification used in the DDB model.

3. The assumptions about the pressure forces, estimated to be proportional to the dynamic pressure and the projected area of the non-distorted droplet, seems to work well, being the proportionality coefficient provided by experimental data 0.93 .

4. Viscosity force seems to have little influence in the final results, so the assumption of a pure extensional flow appears to be accurate enough. 


\section{EXPERIMENTAL VALIDATION OF THE MODEL IN THE STAGNATION LINE IN THE VICINITY OF AN AIRFOIL.}

\subsection{EXPERIMENTAL MATRIX.}

Comparison between the experimental results and those obtained by applying and solving numerically the theoretical model has been carried out for all 45 cases. The summary of the 45 cases selected are provided below:

- Three droplet diameters: $575 \mu \mathrm{m}, 775 \mu \mathrm{m}$, and $1025 \mu \mathrm{m}$

- Three airfoils leading edge radius: $0.103 \mathrm{~m}, 0.070 \mathrm{~m}, 0.030 \mathrm{~m}$.

- Five airfoil velocities: $50 \mathrm{~m} / \mathrm{s}, 60 \mathrm{~m} / \mathrm{s}, 70 \mathrm{~m} / \mathrm{s}, 80 \mathrm{~m} / \mathrm{s}$, and $90 \mathrm{~m} / \mathrm{s}$.

The values of the different dimensionless parameters of the theoretical model $\left(\Pi_{1}, \Pi_{2}\right.$, $\Pi_{3}$, and $\Pi_{4}$, see relations (3.21-3.24)) covered are the following ones:

- $\quad$ Range of $\Pi_{1}: 0.38$ to 14.5

- Range of $\Pi_{2}: 0.05$ to 0.31

- Range of $\Pi_{3}: 0.001$ to 0.072

- Range of $\Pi_{4}: 0.012$ to 2.770

\subsection{RESULTS AND DISCUSSIONS.}

The results obtained for the 8 limiting cases that combine the largest and smallest values of droplet diameter, airfoil leading edge radius, and airfoil velocity are presented $\left(2^{3}=8\right.$ cases) in figures 4.2.1, 4.2.2, 4.2.3 and 4.2.4. It could be observed that, in general, there is a reasonably good agreement between the measured and computed droplet trajectories. Typically, the larger discrepancies tend to occur at the late time instants of the trajectories and, also, for the smaller droplets. The reason could be that in those instants, both the slip velocity and the flow acceleration achieve their largest values and this decreases the accuracy of the experimental droplet tracking system. At the same time, smaller droplets are more difficult to track because they fill in a smaller number of pixels in the camera.

It is important, nevertheless to quantify the discrepancies between the measured and computed droplet trajectories. This has been done for all 45 experimental tests cases. The criterion was, for each case, to compute a figure of merit $\Omega$ defined as the difference (for 
each time) between the experimental and theoretical " $x$ " droplet displacement and to divide it by the maximum experimental value.

$$
\Omega=\left|x_{\text {model }}-x_{\text {exp }}\right| / x_{\text {exp }}
$$

The results of the most critical cases are presented in figure 4.2.5. There, it could be observed that the maximum discrepancy is of the order of $15 \%$ while the bulk of the results show a discrepancy smaller than $6 \%$. The results of the most critical cases are also shown in table 4.2.1. It could be observed that the average discrepancy was of the order of $3.6 \%$.

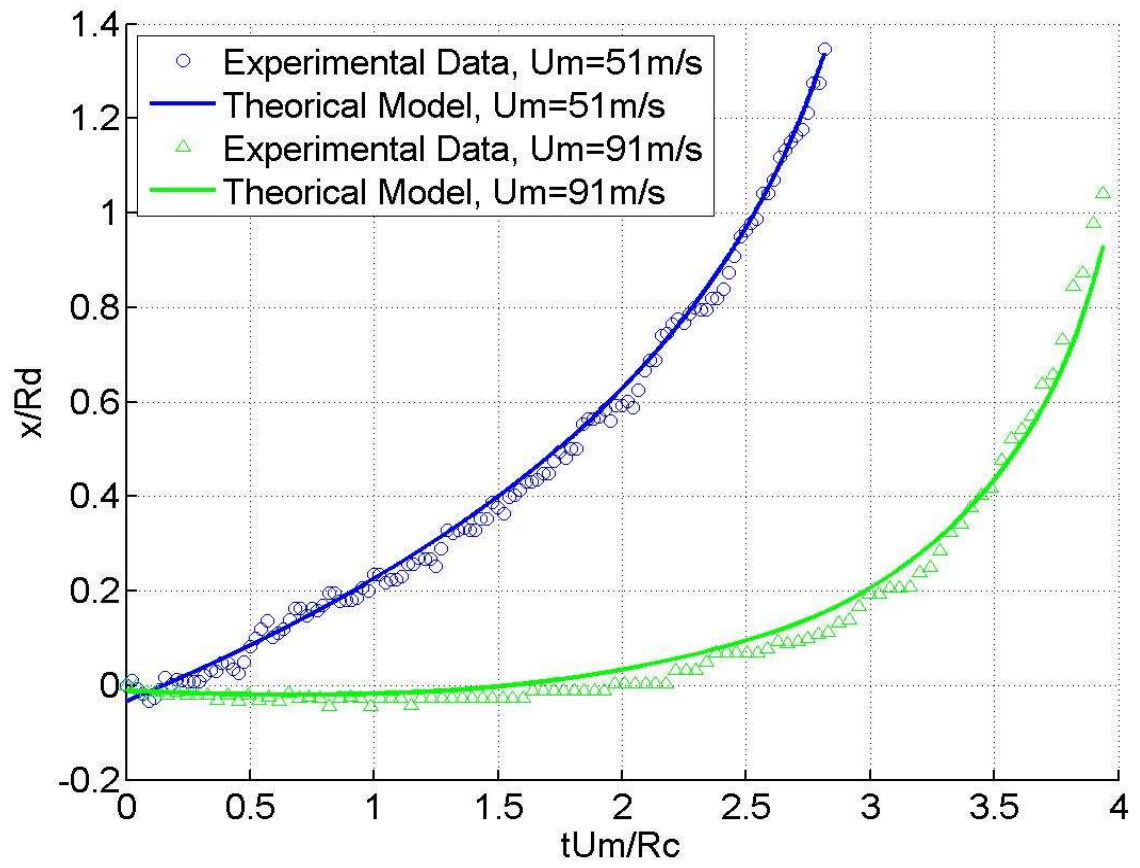

Figure 4.2.1. Comparison between experimental and theoretical results for the case of $R_{c}=0.030 \mathrm{~m}, R_{d}=288 \mu \mathrm{m}$, and two airfoil velocities: $U_{m}=51 \mathrm{~m} / \mathrm{s}$, and $U_{m}=$ $91 \mathrm{~m} / \mathrm{s}$. 


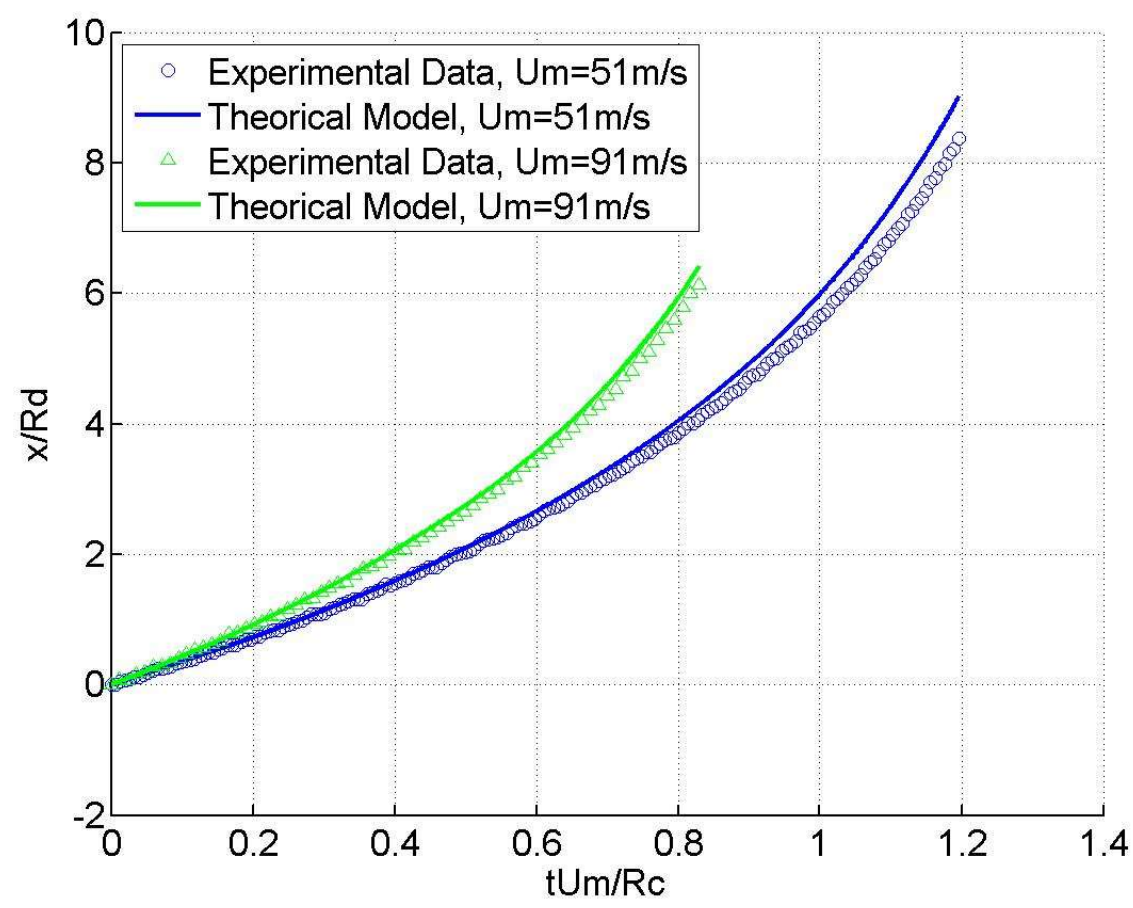

Figure 4.2.2. Counterpart of figure 4.2.1 for $R_{c}=0.103 \mathrm{~m}, R_{d}=288 \mu \mathrm{m}$, and $U_{m}=$ $51 \mathrm{~m} / \mathrm{s}$, and $U_{m}=91 \mathrm{~m} / \mathrm{s}$.

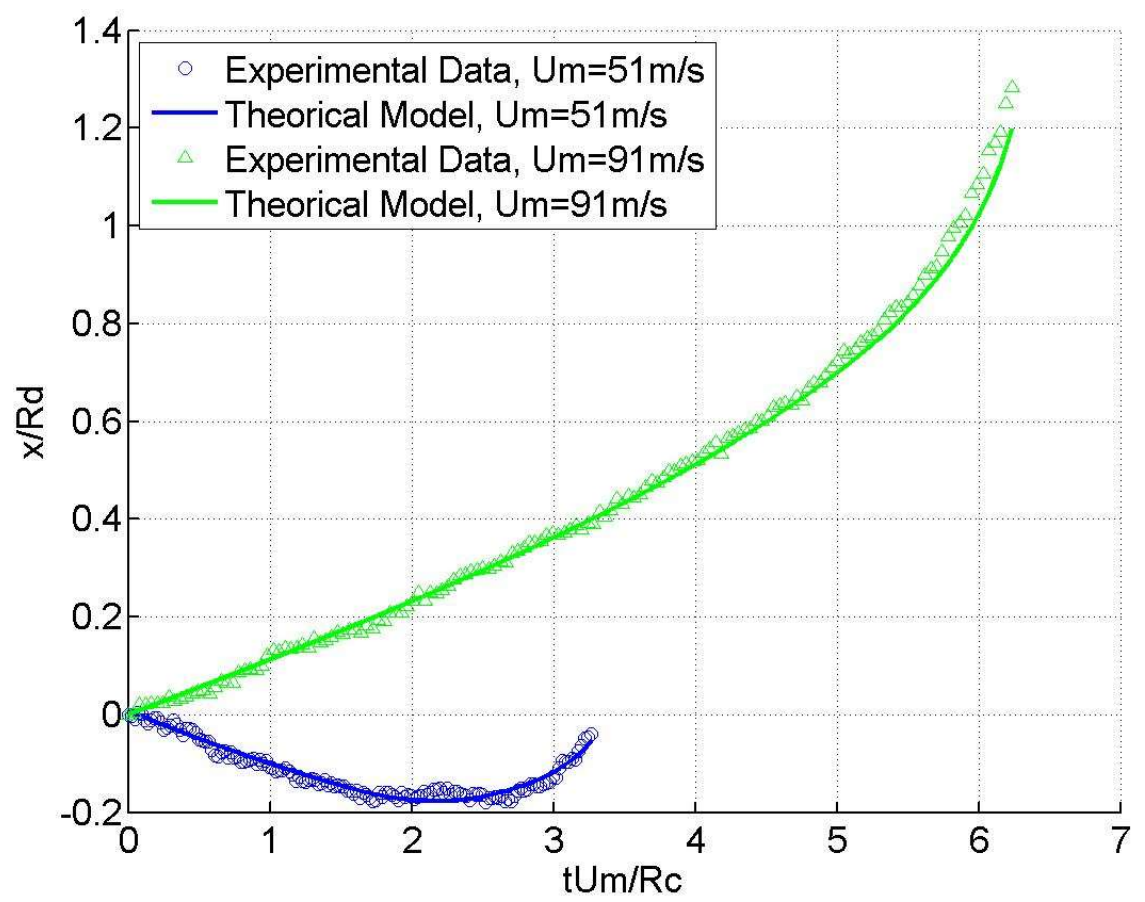

Figure 4.2.3. Counterpart of figure 4.2.1 for $R_{c}=0.030 \mathrm{~m}, R_{d}=513 \mu \mathrm{m}$, and $U_{m}=$ $51 \mathrm{~m} / \mathrm{s}$, and $U_{m}=91 \mathrm{~m} / \mathrm{s}$. 


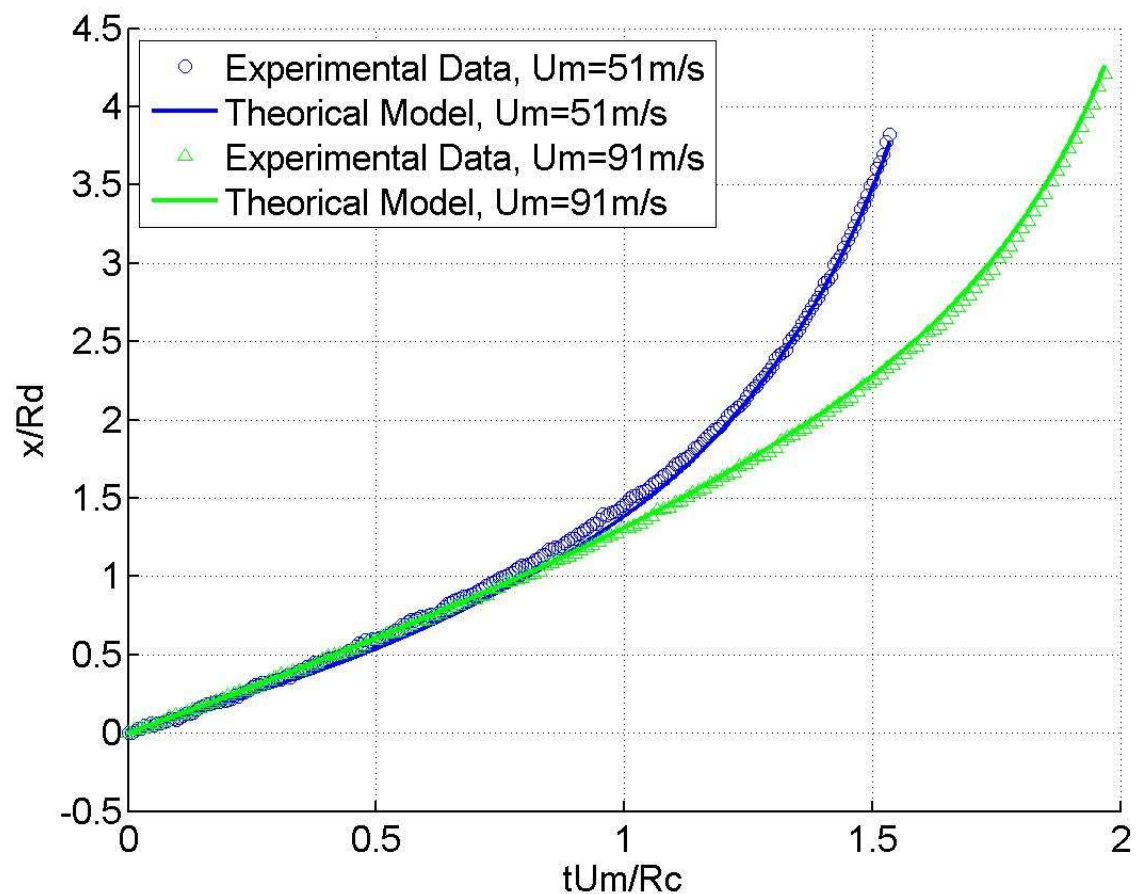

Figure 4.2.4. Counterpart of figure 4.2.1 for $R_{c}=0.103 \mathrm{~m}, R_{d}=513 \mu \mathrm{m}$, and $U_{m}=$ $51 \mathrm{~m} / \mathrm{s}$, and $U_{m}=91 \mathrm{~m} / \mathrm{s}$.

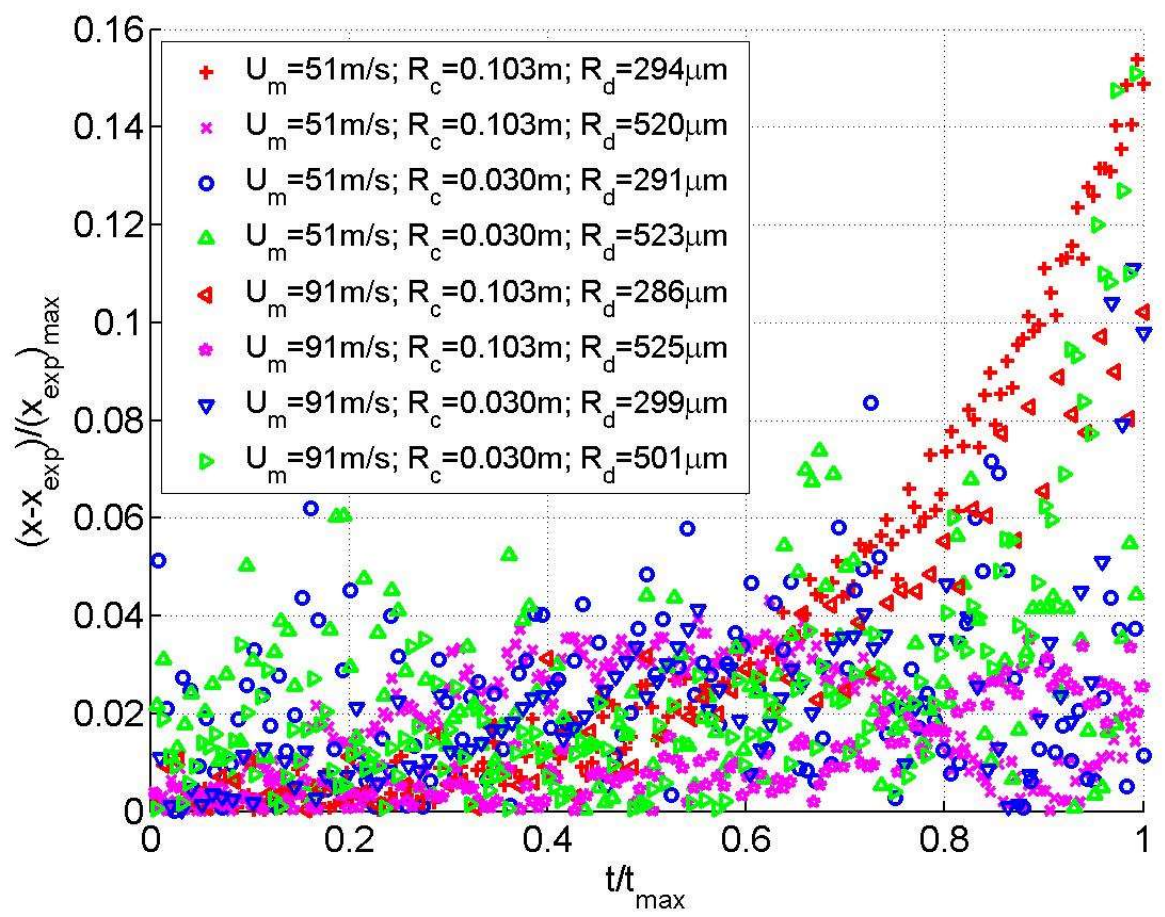

Figure 4.2.5. Discrepancy between measured and theoretical droplet trajectories for the 8 limiting cases presented in figures 4.2.1 to 4.2.4. The figure of merit to quantify the discrepancy is $\left|x_{\text {model }}-x_{\text {exp }}\right| / x_{\text {exp }}$. 


\begin{tabular}{ccccc}
\hline Model & $\mathrm{Um}(\mathrm{m} / \mathrm{s})$ & $\mathrm{Rd}(\mu \mathrm{m})$ & $\operatorname{Mean}(\Omega)$ & $\operatorname{Std}(\Omega)$ \\
\hline M1 & 51 & 294 & 3.64 & 3.99 \\
M1 & 51 & 520 & 1.98 & 1.14 \\
M3 & 51 & 291 & 2.54 & 1.76 \\
M3 & 51 & 523 & 2.54 & 1.69 \\
M1 & 91 & 286 & 2.78 & 2.68 \\
M1 & 91 & 525 & 1.00 & 0.88 \\
M3 & 91 & 299 & 2.17 & 1.85 \\
M3 & 91 & 501 & 2.55 & 2.83 \\
\hline
\end{tabular}

Table4.2.1.Discrepancy between measured and theoretical droplet trajectories for the 8 limiting cases presented in figures 4.2.1 to 4.2.4

Now, it is shown how the droplet deformations obtained from the theoretical model compares to the measured ones. This comparison has been done superimposing on the droplet pictures obtained from the experimental tracking system (dark shade) the computed spheroid cross-section (drawn using a white line). Regarding this photograph composition, a pixel-wise drawing has been selected instead of using a line with continuous derivatives. The results for cases $R_{c}=0.103 \mathrm{~m}, R_{d}=513 \mu \mathrm{m}$, and $U_{m}=$ $91 \mathrm{~m} / \mathrm{s}$ and $R_{c}=0.070 \mathrm{~m}, R_{d}=388 \mu \mathrm{m}$, and $U_{m}=70 \mathrm{~m} / \mathrm{s}$, are presented in figure 4.2.6. 

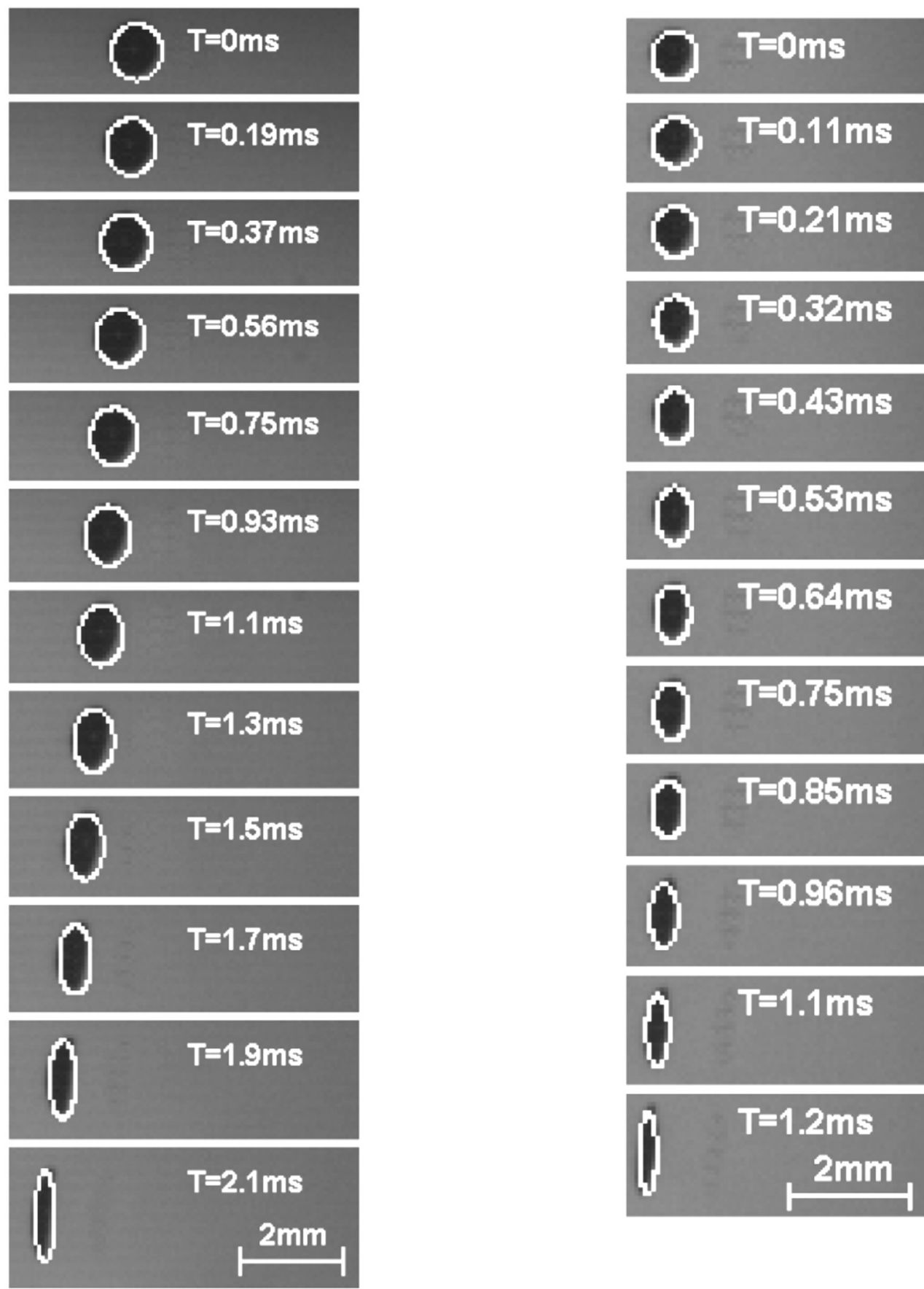

Figure 4.2.6. Comparison between photographed droplet shapes (dark shade) and computed cross-sections (white lines) as a function of time for cases: $R_{c}=0.103 \mathrm{~m}$, $R_{d}=513 \mu \mathrm{m}$, and $U_{m}=91 \mathrm{~m} / \mathrm{s}(\mathrm{left})$, and $R_{c}=0.070 \mathrm{~m}, R_{d}=388 \mu \mathrm{m}$, and $U_{m}=$ $70 \mathrm{~m} / \mathrm{s}$ (right).

It is also possible to superimpose in the pictures of figure 4.2.6 the theoretical droplet trajectories. This is done in figure 4.2.7 where a reasonable agreement can be observed (it wasn't done in figure 4.2.6 because the snapshots shown would overlap if plotted in the same spatial frame of reference). 

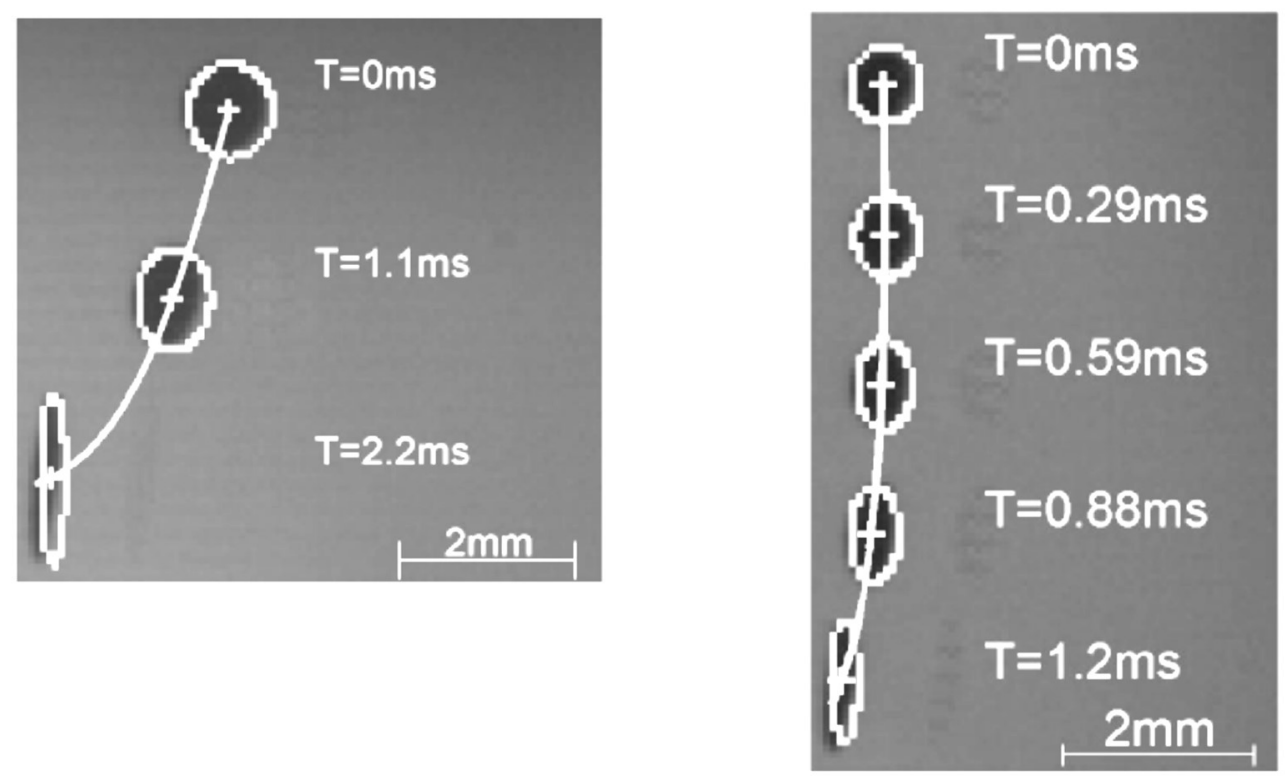

Figure 4.2.7. Counterpart of figure 4.2.6 with the theoretical droplet trajectories superimposed (white lines).

Now, it remains to be evaluated the issue of the relative importance of the quasi-steady, $C_{D 1}$, and unsteady, $C_{D 2}$, components of the drag coefficient in relations (3.5-3.7). To this end, both components have been plotted in figure 4.2.8 for three representative cases. It could be observed that, always, the quasi-steady component is larger than the unsteady one but the latter cannot be neglected (if accurate results are sought) because it is, typically, of the order of $25 \%$ of the former. This means that, at least in the parametric space of parameters that have been considered in this experimental campaign, the unsteady component of the drag coefficient should not be neglected. It is also of interest to plot the total drag coefficient $\left(C_{D}=C_{D 1}+C_{D 2}\right)$ as a function of the instantaneous Reynolds number that the droplet senses. This is done in figure 4.2.9 where the drag coefficient of an equivalent stationary sphere is also plotted for comparison purposes. As it could be observed, in the three cases that are presented in figure 4.2.9 (that are representative of the whole set of experimental cases that have been addressed in this study), the total drag coefficient is always larger than the steady one. Also, the tendency is also different because the total $C_{D}$ grows along with the Reynolds number while the steady sphere $\mathrm{C}_{\mathrm{D}}$ decreases. 


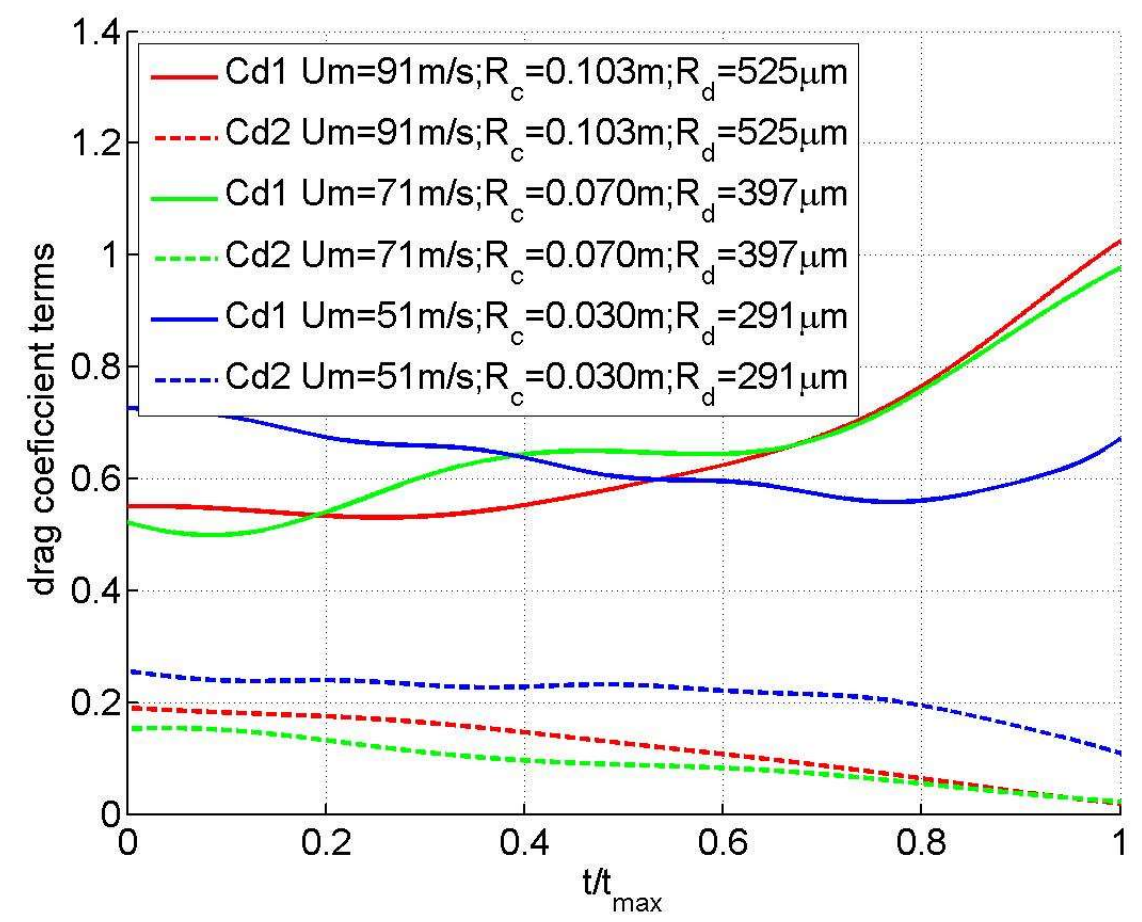

Figure 4.2.8. Comparison between the quasi-steady, $C_{D 1}$, and unsteady, $C_{D 2}$, components of the drag coefficient in relations (3.5-3.7) for three representative cases

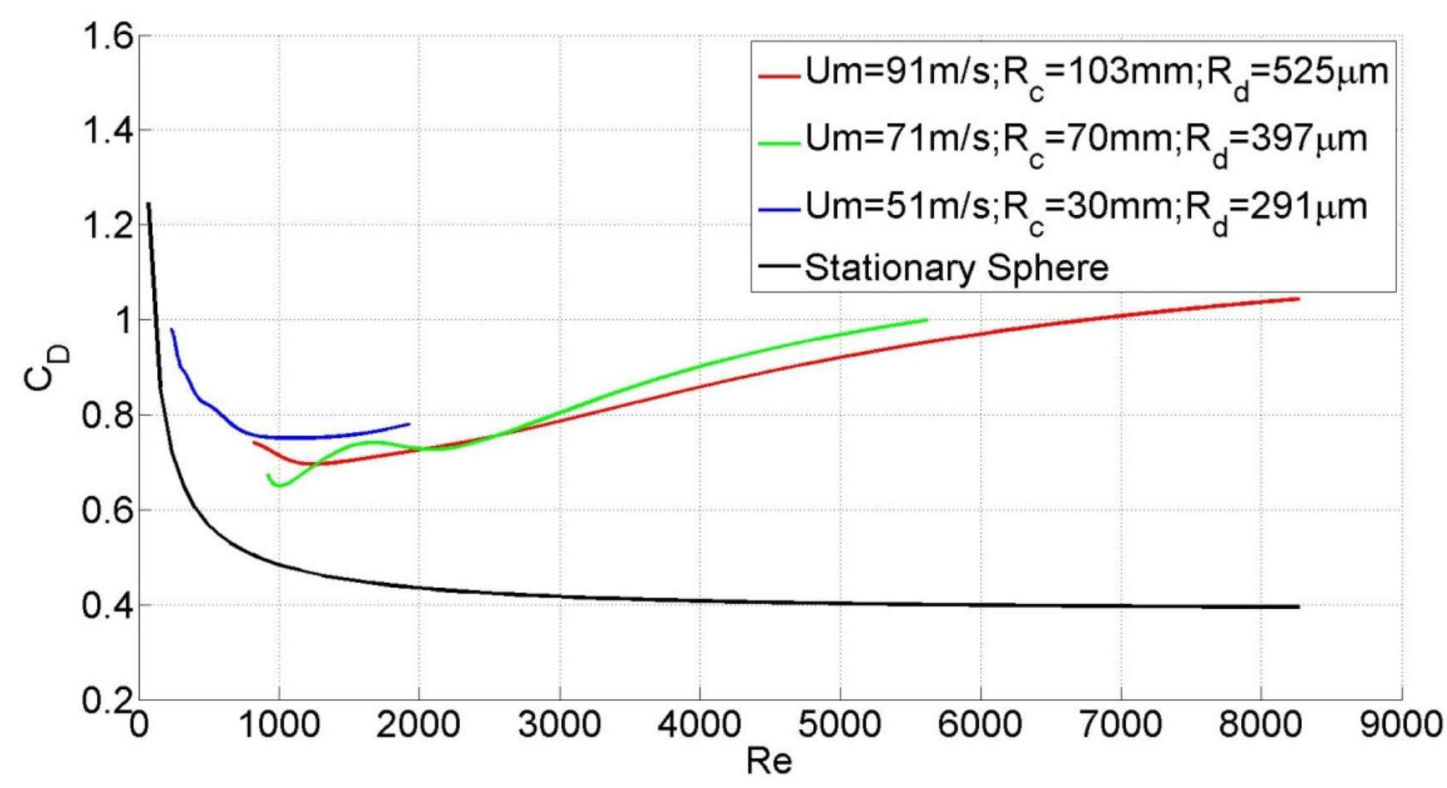

Figure 4.2.9. Drag coefficient versus instantaneous Reynolds number for three different experimental cases. The drag coefficient of the stationary sphere is included for comparison purposes. 
Finally, and for the sake of completion, the three terms in the right hand side of equation (3.3) are plotted separately to allow for comparison and, also, to check the hypothesis that has been made that the viscous force is much smaller than the surface tension force and the pressure force. To this end, once a representative case has been computed (the case parameters were $R_{c}=0.103 \mathrm{~m}, R_{d}=513 \mu \mathrm{m}$, and $U_{m}=91 \mathrm{~m} / \mathrm{s}$ ) it solution was used to compute a posteriori the viscous force. The comparison between the three terms is shown in figure 4.2.10 where it could be observed that the results obtained are consistent with the hypothesis that has been made in equation (3.3).

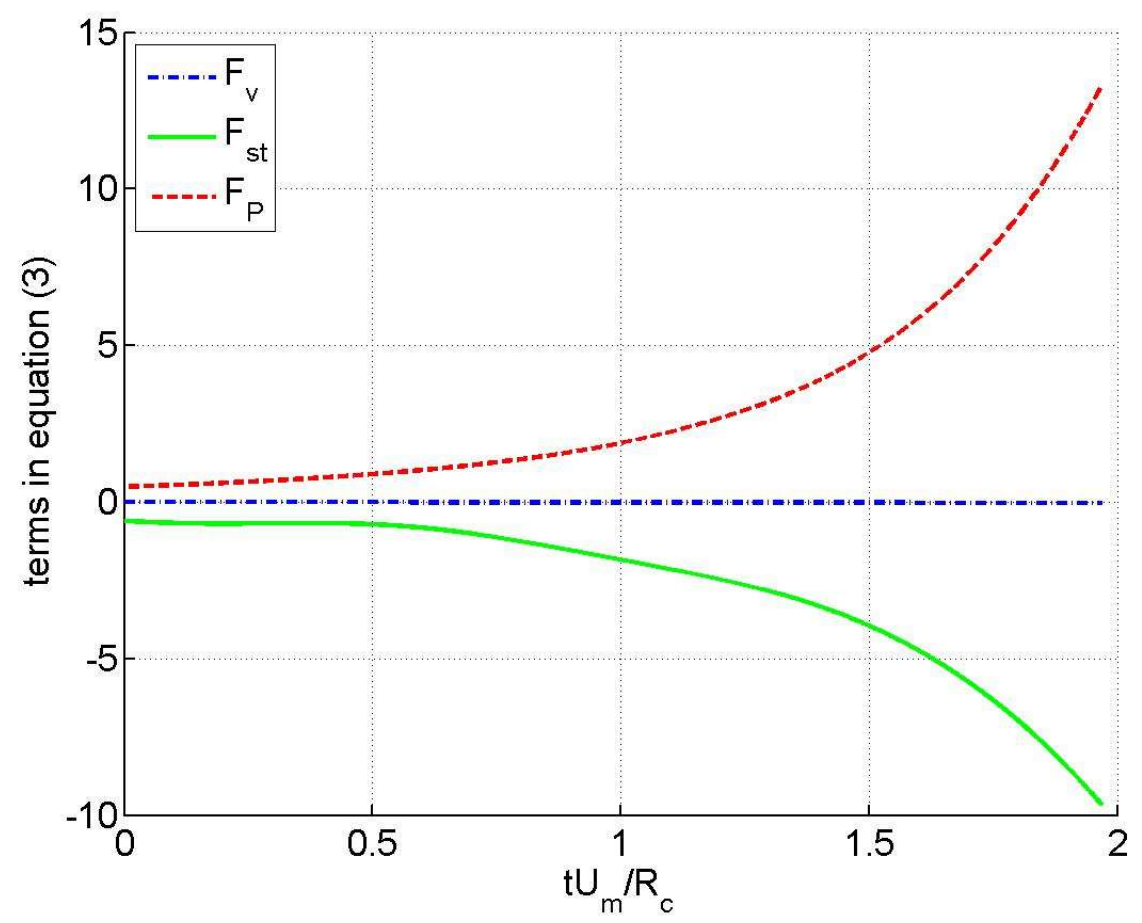

Figure 4.2.10. Evaluation of terms in equation (3.3) for the case $R_{c}=0.103 \mathrm{~m}, R_{d}=$ $513 \mu \mathrm{m}$, and $U_{m}=91 \mathrm{~m} / \mathrm{s}$.

\subsection{CONCLUSIONS.}

A theoretical model has been presented that predicts droplet trajectory and deformation in the vicinity of an incoming airfoil. The model is of interest both to gain a better understanding of droplet trajectories in some flows of aeronautics interest, and to provide an alternate formulation to researchers that develop numerical flow solvers in the field of icing conditions simulations. The model has been tested and calibrated using a series of dedicated experimental tests in a rotating arm facility. The novelty of the study consists on the fact that it accounts for the actual flow acceleration profile that the droplet senses as the airfoil closes on it. This is contrast to other more basic-physics oriented studies in which shock tube type no acceleration profiles are considered. The conclusions that have been obtained are, basically, two. 
First, it is worth mentioning that a relatively simple model based on droplet dynamics and deformation (three ordinary differential equations) is sufficient to predict with reasonable accuracy droplet trajectory in the conditions that have been considered. The model can be said to have a "global" character because it implements force formulations that are "global" themselves. For example, the pressure force does not depend on the actual local coordinate on the droplet surface but it is modeled after a "global" coefficient. This suggests that the two problems of: a) the onset and further development of flow instabilities leading to droplet deformation and eventual break-up, and b) computation of droplet trajectory, are loosely coupled only. That is, once the conditions that define the basic type of deformation and break-up are set, the droplet trajectory can be modeled somewhat independently. For example, in the parametric range of cases that have been considered, assuming that the droplet deforms following the shape of a spheroid appears to be enough for trajectory prediction purposes.

The second question to be noted is that in the cases that have been addressed in the experimental campaign, implementation of an unsteady term in the aerodynamics drag model (the so-called acceleration parameter) is essential to allow for model accuracy. The issue of separating the drag into quasi-steady and unsteady parts (each one with its own modelization) is somewhat controversial and no claim is made in this thesis regarding its generalization. The only thing that can be said is that for the range of parameters under consideration in the present experimental campaign, the unsteady part of the drag coefficient was critically needed to compute trajectories that compared well to the observed trajectories. Regarding the unsteady drag model itself, the main differences between the model proposed in this article and previous results put forward by other researchers are two:

a) In the present model the acceleration parameter includes, also, the time dependent droplet dimension in the slip velocity-wise direction. This parameter was not considered in formulations described previously in the literature. In this sense, it could be said that its inclusion here makes sense because of the fact that droplets deformed significantly in these rotating arm experiments. It is to be noted that in other experimental studies researchers sometimes used droplets manufactured on materials (plastic type) that did not allow for large deformations. On the Fluid Mechanics side, the reason for the implementation of the unsteady drag term is based on the fact that in an accelerating (or decelerating flow) the size of the recirculation region downstream of the droplet changes continuously; and the dynamics of this change affect the evolution of the drag. Then, in this regard, it is sensible to modify the acceleration parameter so as to account for information on droplet continuous deformation in the slip velocity-wise direction that also affects the dynamics of the recirculation region. 
b) The coefficient that multiplies the modified acceleration parameter in the present model is 9 . In other studies dealing with different experimental setups, researchers found that this coefficient was dependent, also, on some other parameter(s) of the problem. However, in the present study it was found that for the range of parameters under consideration, the coefficient was always 9.

Finally, it should be said that the previous conclusions only apply for the cases that consider the flow acceleration profile generated by an incoming airfoil. This means that these conclusions cannot be extrapolated to other situations. A systematic study on the influence that functionally different acceleration profiles may have on droplet deformation and droplet trajectory is out of the scope of the present study. 


\section{APLICABILITY OF THE MODEL TO PREDICT THE TRAJECTORY OF DROPLETS OUTSIDE THE STAGNATION LINE OF AN AIRFOIL.}

In the previous chapter the experimental study on droplet deformation in the leading edge region (close to the stagnation streamline) of an airfoil conducted was presented. However, that study was limited in the sense that to generate reliable prediction tools, knowledge on the phenomenon all around the airfoil is required. This is why the present study in this chapter focuses, complementarily, on droplet deformation in the shoulder region of the airfoil. From the Fluid mechanics point of view, the main difference with the case presented previously is that in the airfoil leading edge region the flow, as seen from the droplet frame of reference, accelerates in the direction of the airfoil motion with no significant gradient in the direction normal to it. However, in the shoulder region, there are velocity gradients in the direction of the airfoil motion and, also in the direction normal to it. As it will be shown in a later section, this fact gives rise to a different sequence of the droplet deformation behavior. Finally, it is to be noted that the author of this thesis was not able to find previously published experimental results on droplet deformation in the shoulder region of an airfoil; so, to the best of their knowledge, the present results might be the first to be available in the open literature.

The work presented in this chapter has been submitted to Journal of Aerospace Engineering in 2016.

\subsection{TESTS MATRIX.}

Nine different cases were addressed in this experimental study. They corresponded to the variation of two parameters:

- Parameter \#1: the velocity of the approaching airfoil. Three cases were considered: $50 \mathrm{~m} / \mathrm{s}, 70 \mathrm{~m} / \mathrm{s}$ and $90 \mathrm{~m} / \mathrm{s}$.

- Parameter \# 2: the position relative to the airfoil of the field of vision (FOV) where the droplet deformation was recorded. Three different heights of the mid-line of the FOV with respect to the airfoil stagnation line were considered: FOV \#1: 38 $\mathrm{mm}, \mathrm{FOV} \# 2$ : $58 \mathrm{~mm}$, and FOV \#3: $66 \mathrm{~mm}$. 
The initial droplet diameter (on the order of $1 \mathrm{~mm}$ ) and the airfoil model were unchanged through the experiments. To ensure repeatability, each of the nine experimental cases was repeated four times. To illustrate the area that was analyzed, the position of the three different FOV's is shown in figure 5.1.1. A sketch of the typical results to be expected for each FOV is shown in figure 5.1.2.

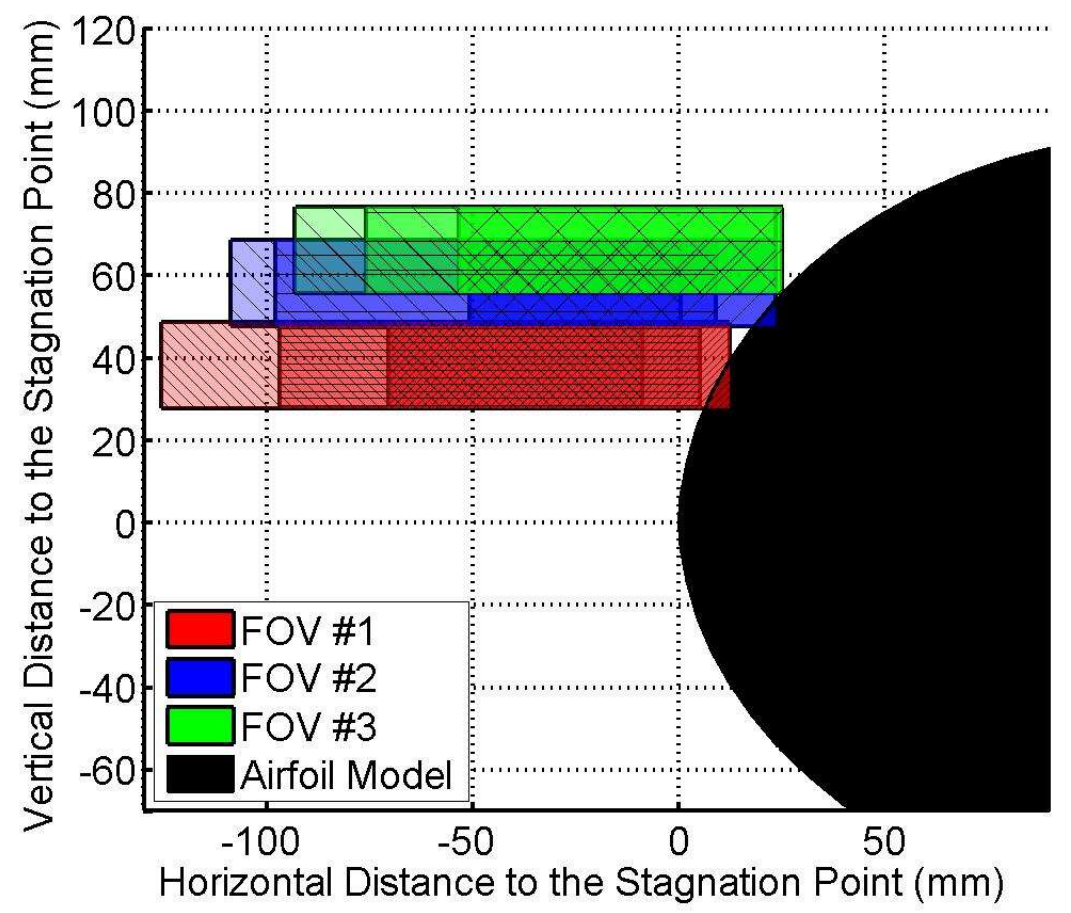

Figure 5.1.1. Location of the different Fields of View (FOV) relative to the airfoil 


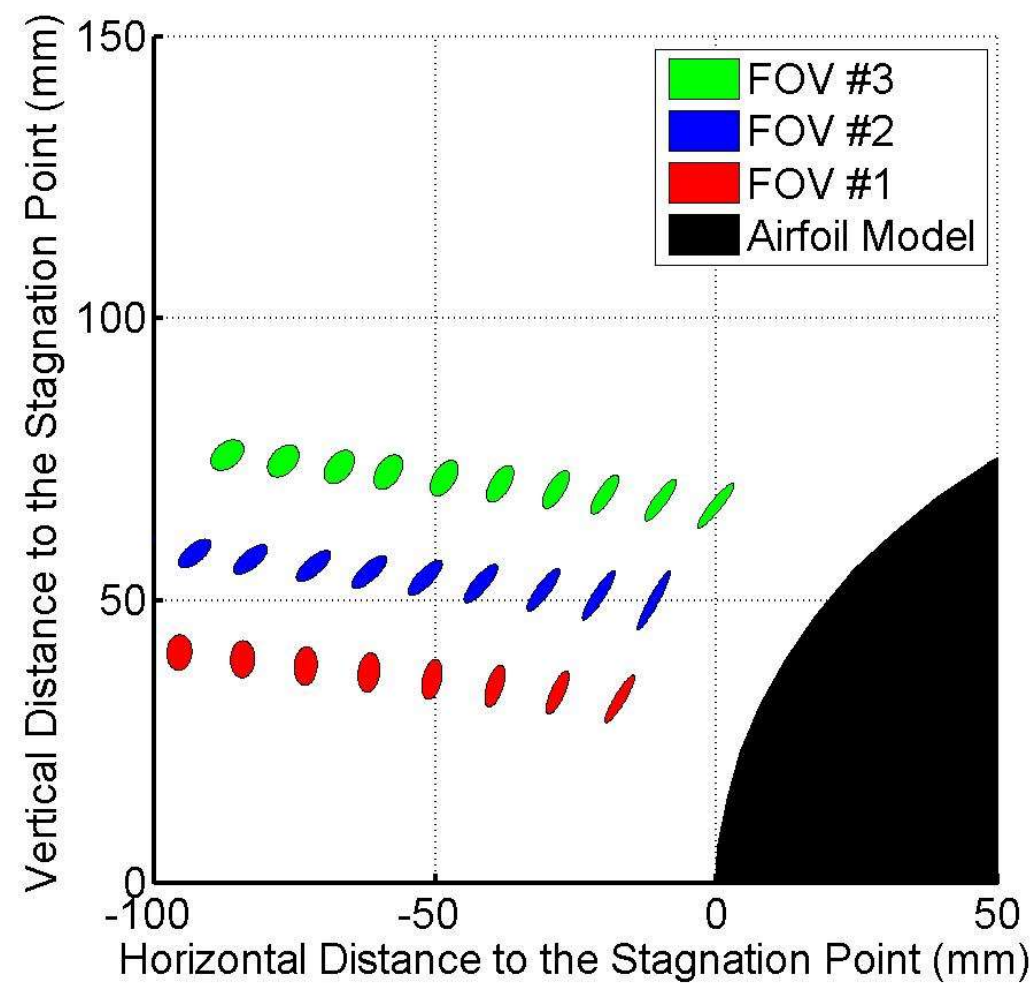

Figure 5.1.2. Sketch of the type of results to be expected relative to the airfoil

The key for the nine different cases is presented in table 5.1.1.

\begin{tabular}{cccc}
\hline Case Key & FOV \#1 & FOV \#2 & FOV \#3 \\
\hline $\mathrm{U}_{\mathrm{m}}=50 \mathrm{~m} / \mathrm{s}$ & 1 & 2 & 3 \\
$\mathrm{U}_{\mathrm{m}}=70 \mathrm{~m} / \mathrm{s}$ & 4 & 5 & 6 \\
$\mathrm{U}_{\mathrm{m}}=90 \mathrm{~m} / \mathrm{s}$ & 7 & 8 & 9 \\
\hline
\end{tabular}

Table 5.1.1. Key of the nine different experimental cases. $U_{\mathrm{m}}$ stands for the airfoil velocity.

\subsection{PARTICULARITIES OF THE STUDY REGION.}

From a quantitative point of view, each of the nine cases described in Table 5.1.1 is characterized by the slip velocity profile that the airfoil generates past the droplet. In particular, it is the loading associated to the velocity profile what causes droplet deformation and eventual breakup. Then, the nine slip velocity profiles have been measured and the results are presented in figures 5.2.1, 5.2.2, and 5.2.3 as a function of the horizontal distance to the airfoil leading edge. 


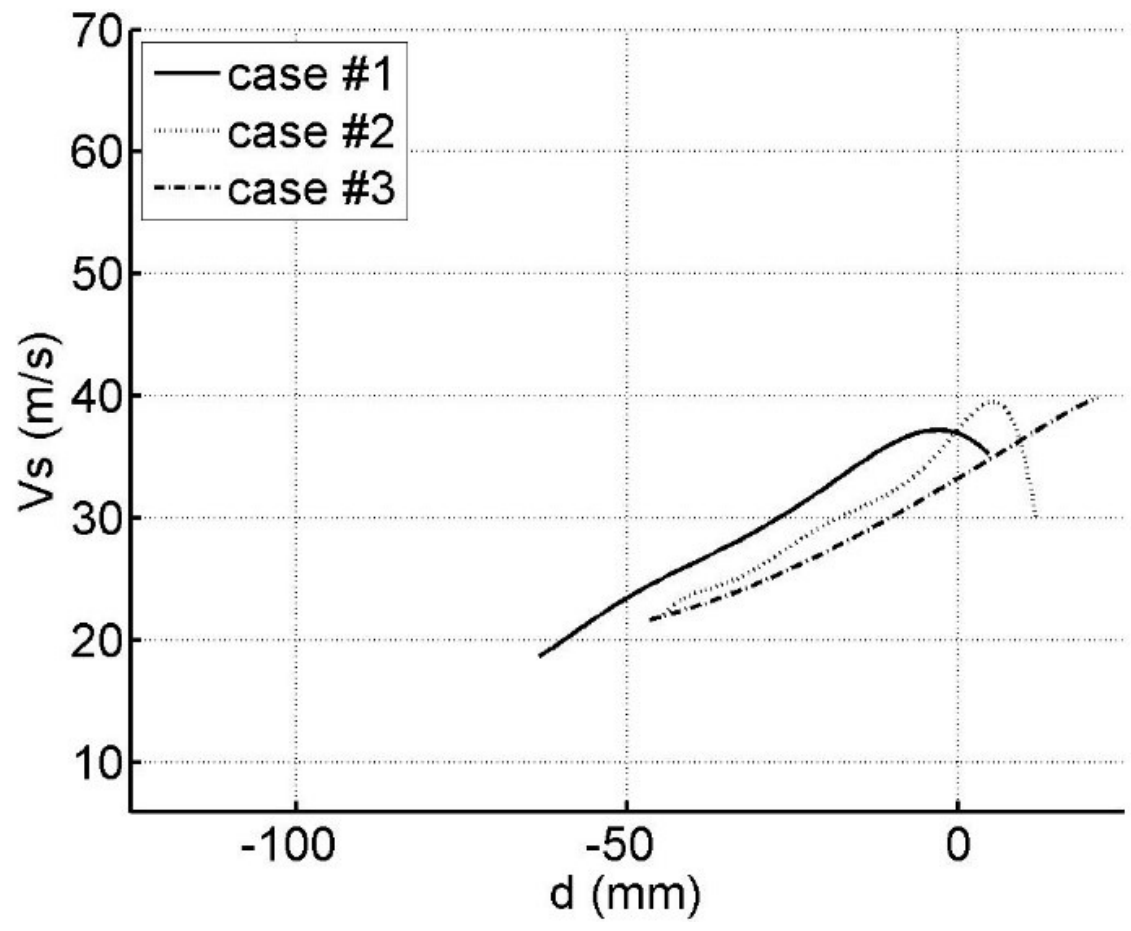

Figure 5.2.1. Slip velocity profiles for cases $\# 1, \# 2$, and $\# 3$ as a function of the droplet horizontal distance to the airfoil leading edge.

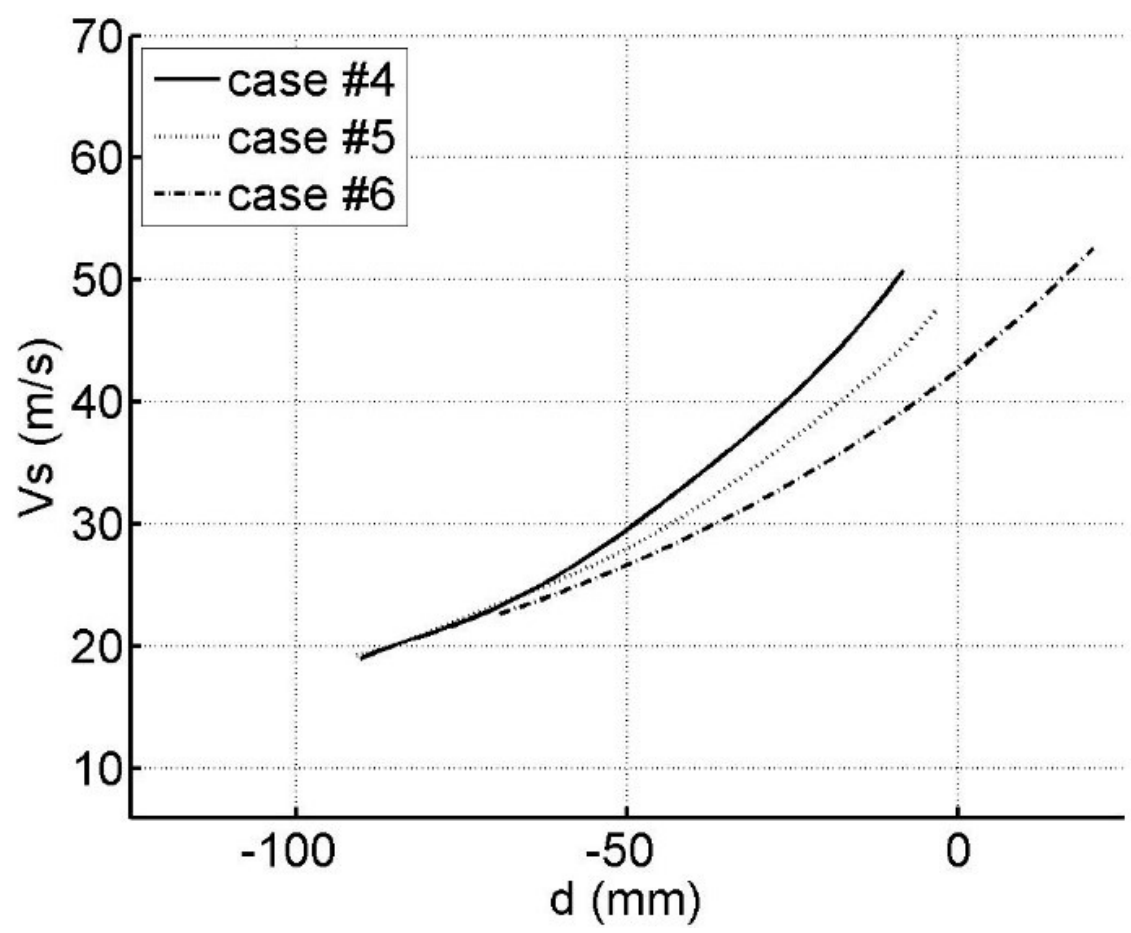

Figure 5.2.2. Slip velocity profiles for cases \#4, \#5, and \#6 as a function of the droplet horizontal distance to the airfoil leading edge. 


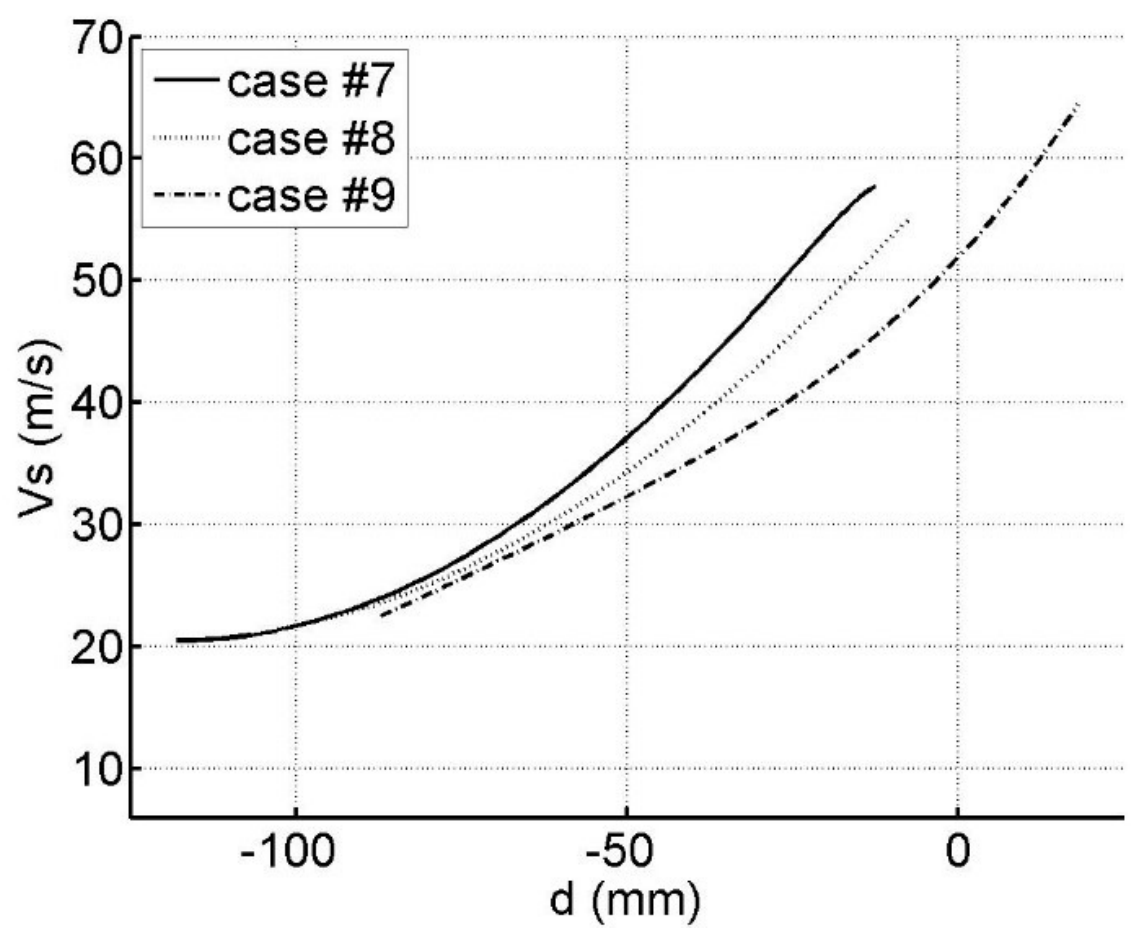

Figure 5.2.3. Slip velocity profiles for cases $\# 7, \# 8$, and $\# 9$ as a function of the droplet horizontal distance to the airfoil leading edge.

Regarding the repeatability of the results, figure 5.2.4 shows the uncertainty band associated to different repetitions of case \#3.

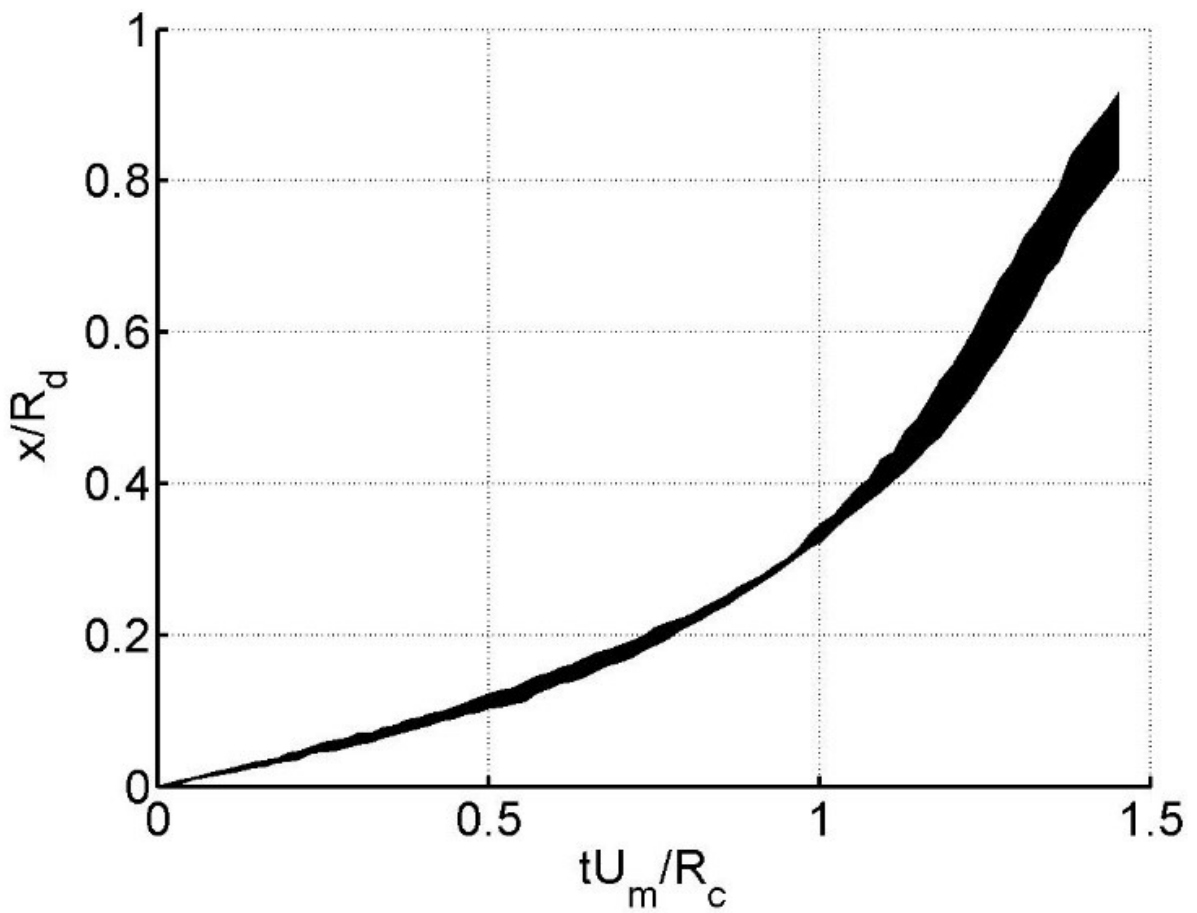

Figure 5.2.4. Uncertainty band associated to the different repetitions of case \#3. 
When looking, for example, at figure 5.2.1 it could be observed that the slip velocity profile associated to FOV \#1 yields velocities that are larger than those associated to FOV \#2. At the same time, slip velocities associated to FOV \#2 are larger than those corresponding to FOV \#3. The reason, see figure 5.1.1, is that FOV \#1 is the closest one to the airfoil leading edge region, while FOV \#3 is the one located furthest away from it. The same trend can also be observed in figures 5.2.2 and 5.2.3. Also, when comparing, for example, figures 5.2.1 and 5.2.2, it could be seen that slip velocities are larger in figure 5.2.2 that correspond to an airfoil velocity of $70 \mathrm{~m} / \mathrm{s}$, than those presented in figure 5.2.1 that correspond to an airfoil velocity of $50 \mathrm{~m} / \mathrm{s}$. The same behavior could be observed when comparing figures 5.2.2 and 5.2.3.

\subsection{EXPERIMENTAL RESULTS AND DISCUSSION.}

Now, the evolution of droplet deformation associated to the nine cases defined in table

5.1.1 is presented in figures 5.3.1, 5.3.2, and 5.3.3. 

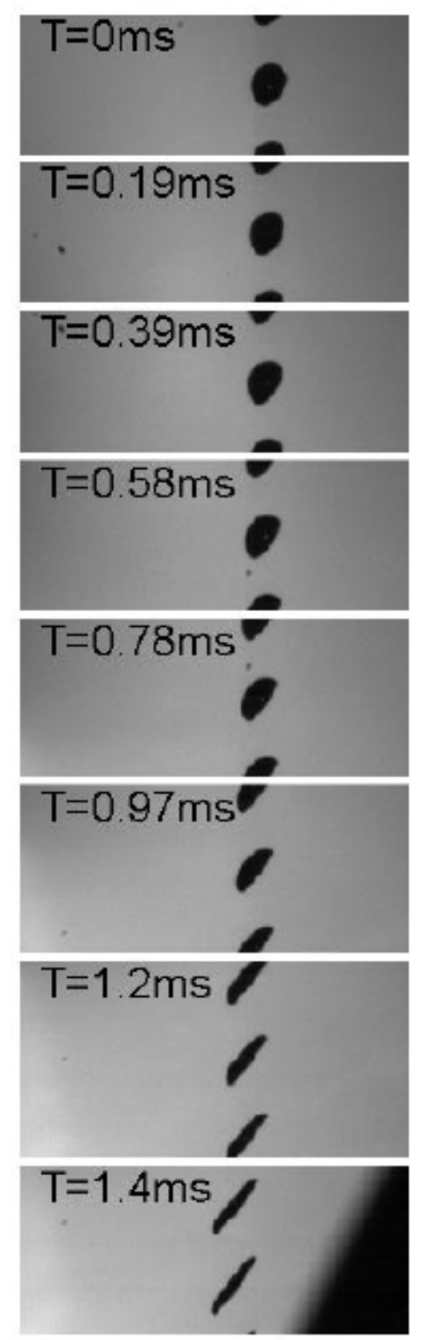
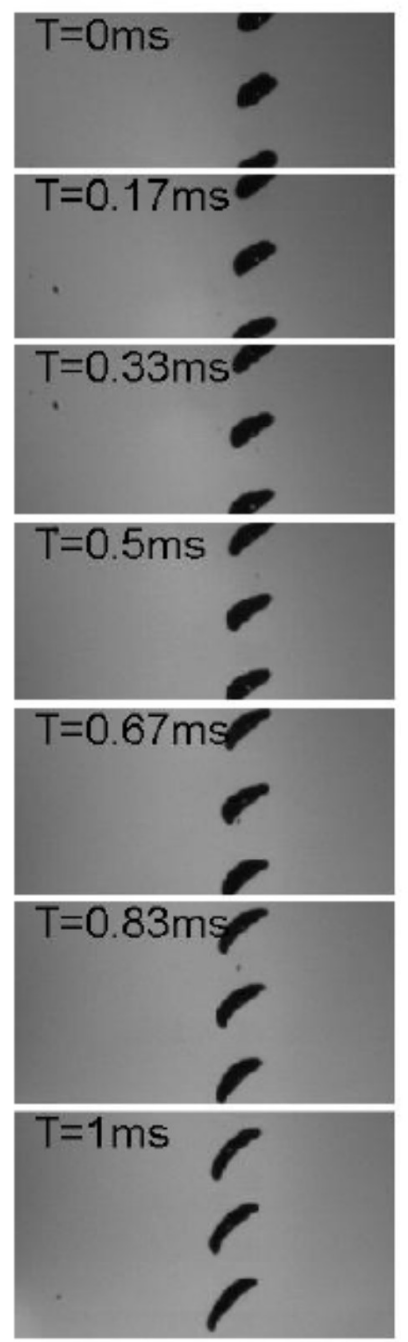
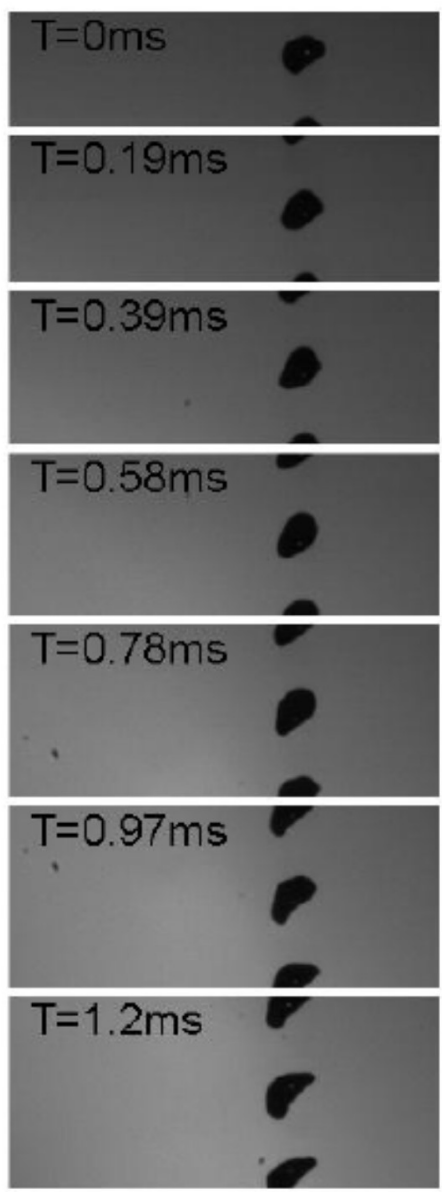

$\mathrm{T}=1.4 \mathrm{~ms}$

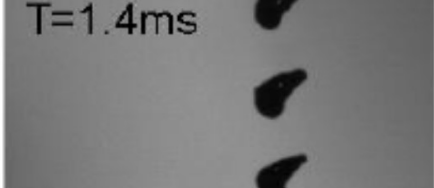

Figure 5.3.1. Droplet Evolution for cases \#1(left), \#2 (centre), and \#3 (right)

Figure 5.3.1 is illustrative in the sense that in this case, that is characterized by the lowest airfoil velocity $(50 \mathrm{~m} / \mathrm{s})$, the droplet deformation (that can be quantified as the ratio of the largest to the smallest dimension) is markedly different depending of the particular FOV. For example, final deformation associated to FOV \#3 is much smaller than deformation associated to FOV \#1. It is also worth observing that the deformed droplets tilt in the direction of the incoming airfoil. Incidentally, the dark shape of the airfoil can be glimpsed in the lowest frame of the left column of figure 5.3.1. When the airfoil velocity increases up to $70 \mathrm{~m} / \mathrm{s}$, see figure 5.3.2, all the deformations tend to be similar. This observation is further reinforced when observing the results presented in figure 5.3.3. The reason could be that above some threshold loading, all droplets tend to deform to the same asymptotic state. It is, nevertheless, worth noting that the local details of the deformation are different even if the global aspects are similar. This can be seen when comparing the right and left columns of figure 5.3.2, and the right and left columns of figure 5.3.3. In those cases, droplets existing in FOV \#3 tend to deform with concavity pointing towards the incoming airfoil, while the opposite happens for droplets recorded inside FOV \#1. 
This suggests that droplet deformation is apt to be analyzed in terms of both local and global patterns. Or to put in other words, a plausible theoretical model of model deformation might have two different scales: the local and the global scale.
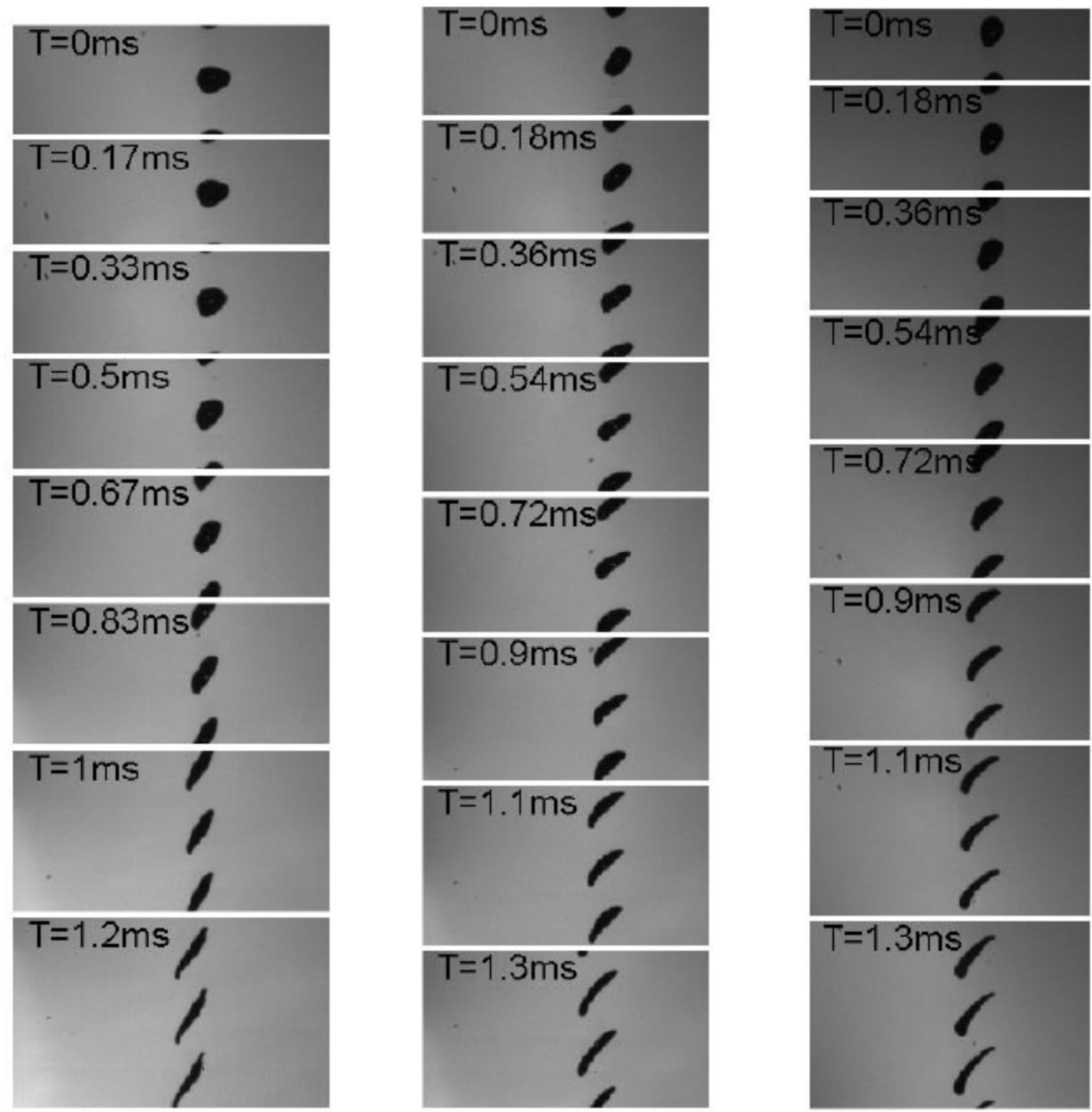

Figure 5.3.2. Droplet evolution for cases \#4 (left), \#5 (centre), and \#6 (right). 

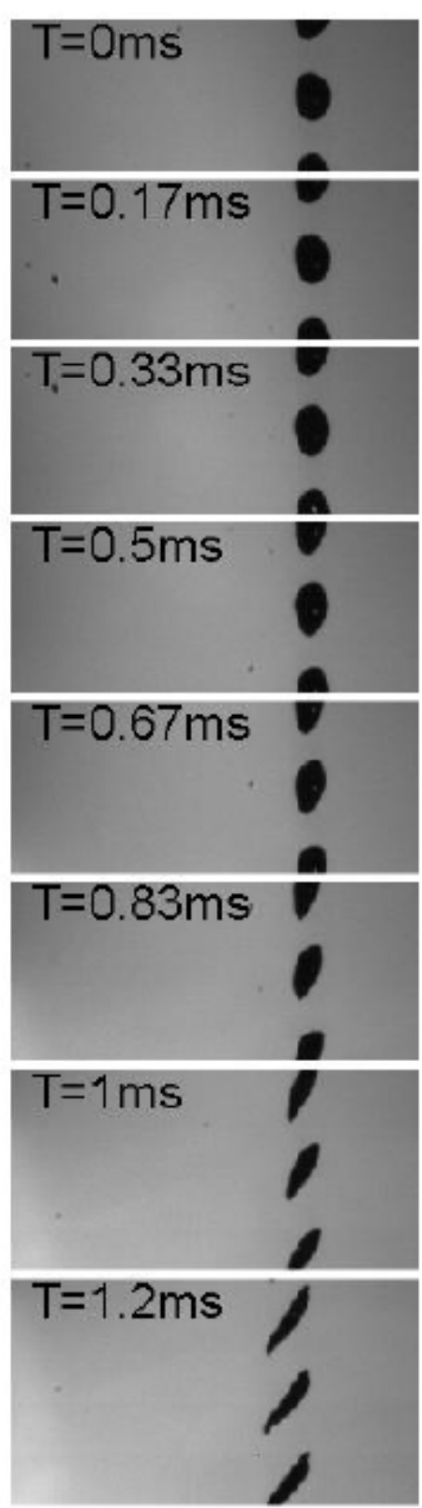
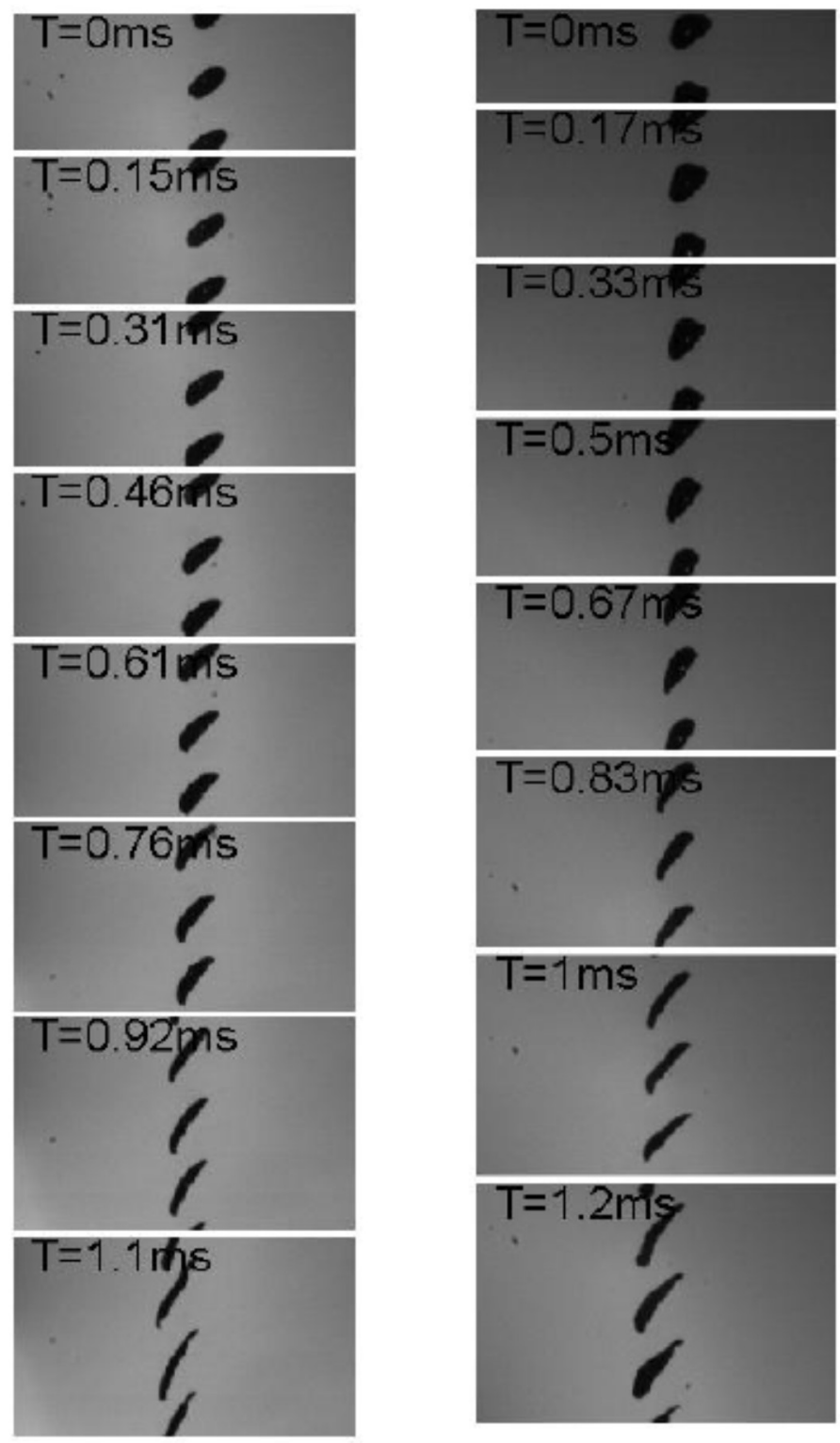

Figure 5.3.3. Droplet evolution for cases \#7 (left), \#8 (centre) and \#9 (right).

Another aspect to be noted in the results presented in figures 5.3.1, 5.3.2, and 5.3.3 is that the deformation of the train of droplets causes aerodynamic interference between the droplets themselves. This is because of the above mentioned tilting towards the incoming airfoil that is in contrast with experimental results obtained for trains of droplets in the leading edge region (see chapter 4). In the present case, the tilting suggests that the aerodynamics behavior of each single droplet is somewhat coupled to that of its nearest neighbors. To further quantify this effect, the slip velocity direction has been computed out of the experimental results and it is shown, superimposed in green color, in the train of droplets corresponding to case \#7, see figure 5.3.4. 


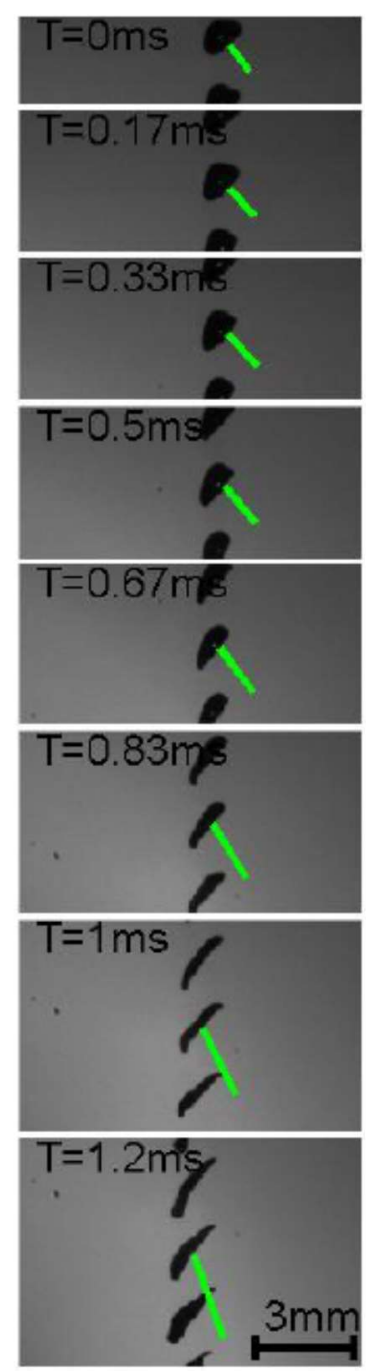

Figure 5.3.4. Direction of the slip velocity, computed from the experimental results, superimposed on the droplet's train corresponding to case \#9.

To observe in more detail this effect, the last snapshot of figure 5.3.4 has been enlarged and it is shown in figure 5.3.5. There, it could observed that each single droplet is partly shielded by their downstream neighbor and possibly affected by its downstream recirculation region; so this fact affects the aerodynamics loading impinging on it. Nevertheless, it is to be noted that the 2D picture of figure 5.3.5 might give an exaggerated impression of the actual shielding. The reason is that in the actual $3 \mathrm{D}$, situation the fact that deformed droplets resemble disks causes the shielding to be smaller (this could have been observed if pictures in the direction perpendicular to that of figure 5.3.4 could have been taken). In any case, all this suggests that, in the shoulder region of the airfoil, analytical models for droplet deformation should not target isolated droplets but, instead, they should account for aerodynamic interferences. In practice, this means that the modeling complexity required for this region may increase. 


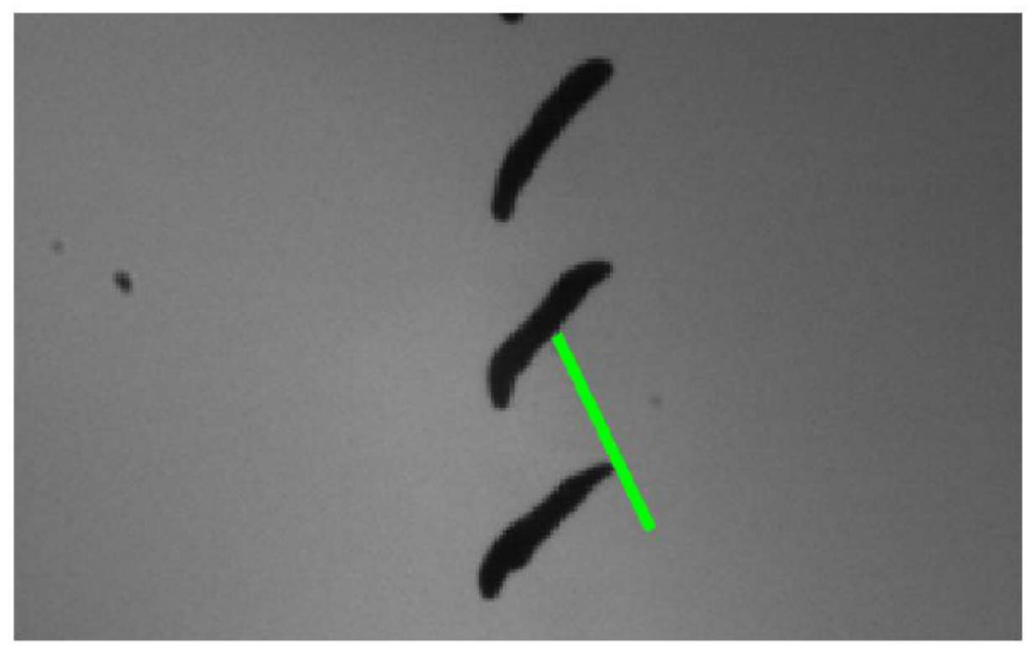

Figure 5.3.5. Close up view of the last snapshot of figure 5.3.4 where the aerodynamic shielding of droplets can be observed in detail (the direction of the slip velocity is drawn in green).

The results presented in figures 5.3.4 and 5.3.5 should be compared to those presented in figure 5.3.6 where the typical evolution of a droplet in the airfoil stagnation region is presented. Again, and to facilitate the comparison, the slip velocity direction has been obtained from the experimental data and it is shown superimposed in green color. When comparing figures 5.3.4 and 5.3.6 it is important to notice the marked difference in droplet tilting towards the airfoil. This tilting, quantified by the inclination of the green line (the direction of the slip velocity) is much smaller in figure 5.3.6 (airfoil leading edge region) than in figure 5.3.4 (airfoil shoulder region). 

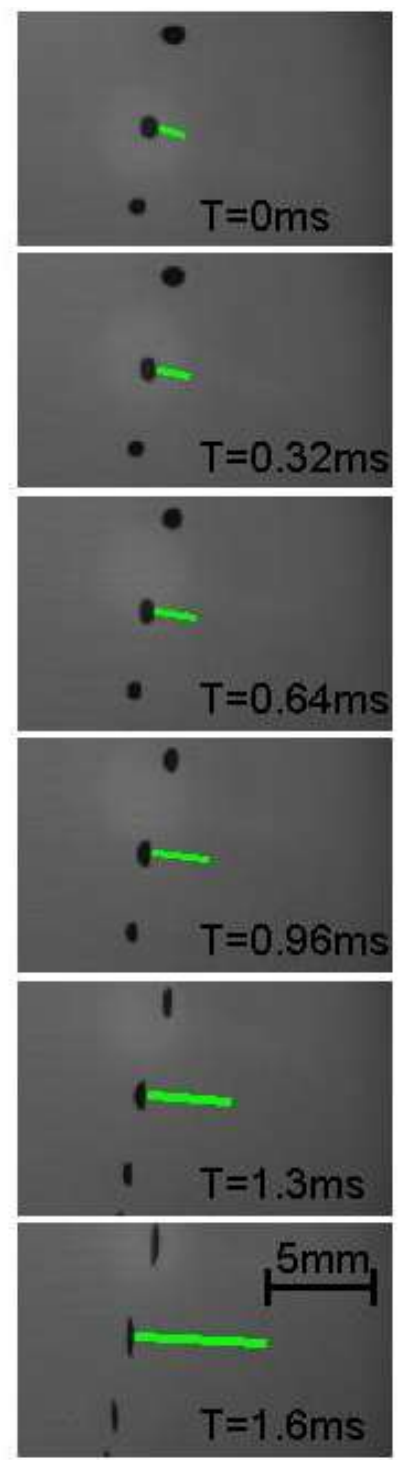

Figure 5.3.6. Counterpart of figure 5.3.4 for droplets in the airfoil leading edge region. The slip velocity is superimposed in green color.

To further ascertain the limitations of existing models, the recorded experimental trajectories of the droplets in all the cases are compared, see figures 5.3.7, 5.3.8 and 5.3.9, to those obtained by integrating the theoretical model presented in the previous chapter. This model was developed to predict droplet deformations and trajectories in the leading edge flow region of the airfoil and, therefore, is not formally well suited to deal with the present situation. In the figures, Rc stands for the leading edge radius of the airfoil and $\mathrm{Rd}$ stand for the initial droplet radius. 


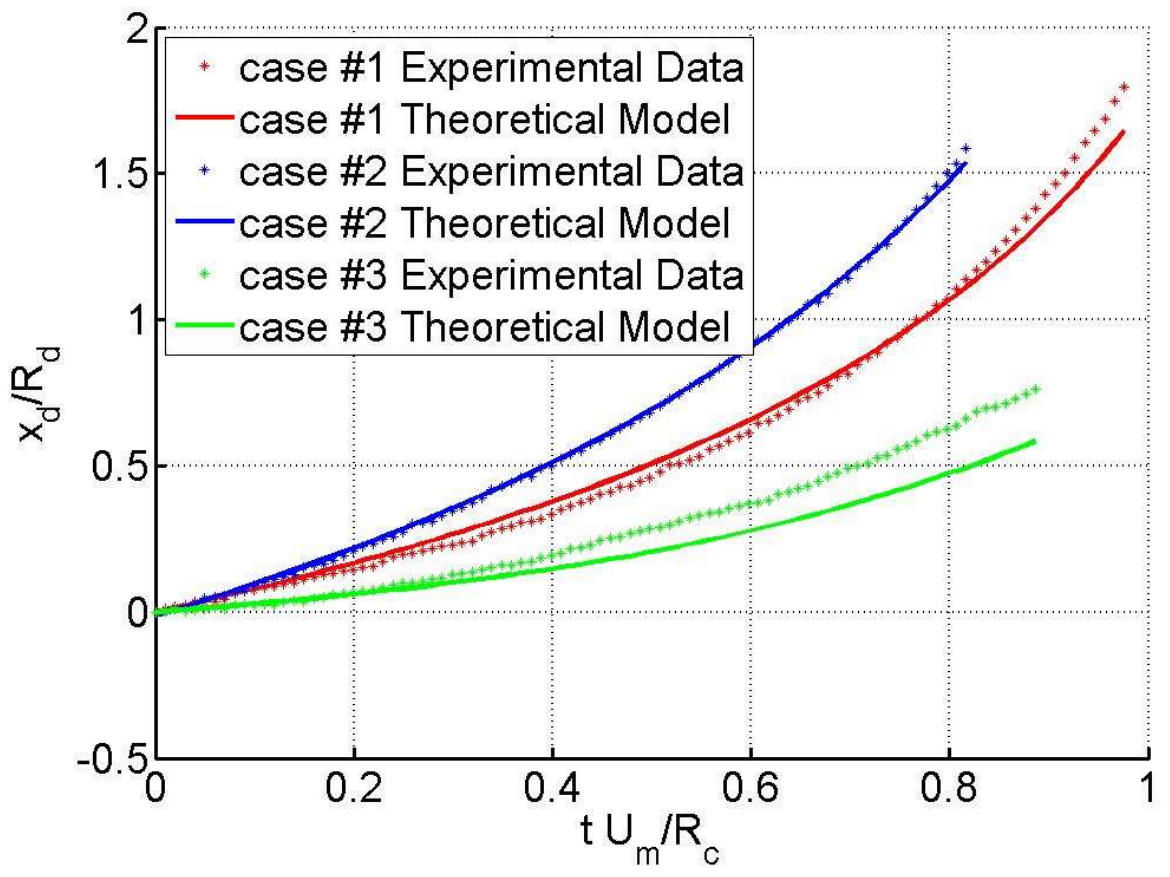

Figure 5.3.7. Comparison of the recorded droplet's horizontal position for cases \#1, \#2, and \#3 with those obtained by integrating the theoretical model.

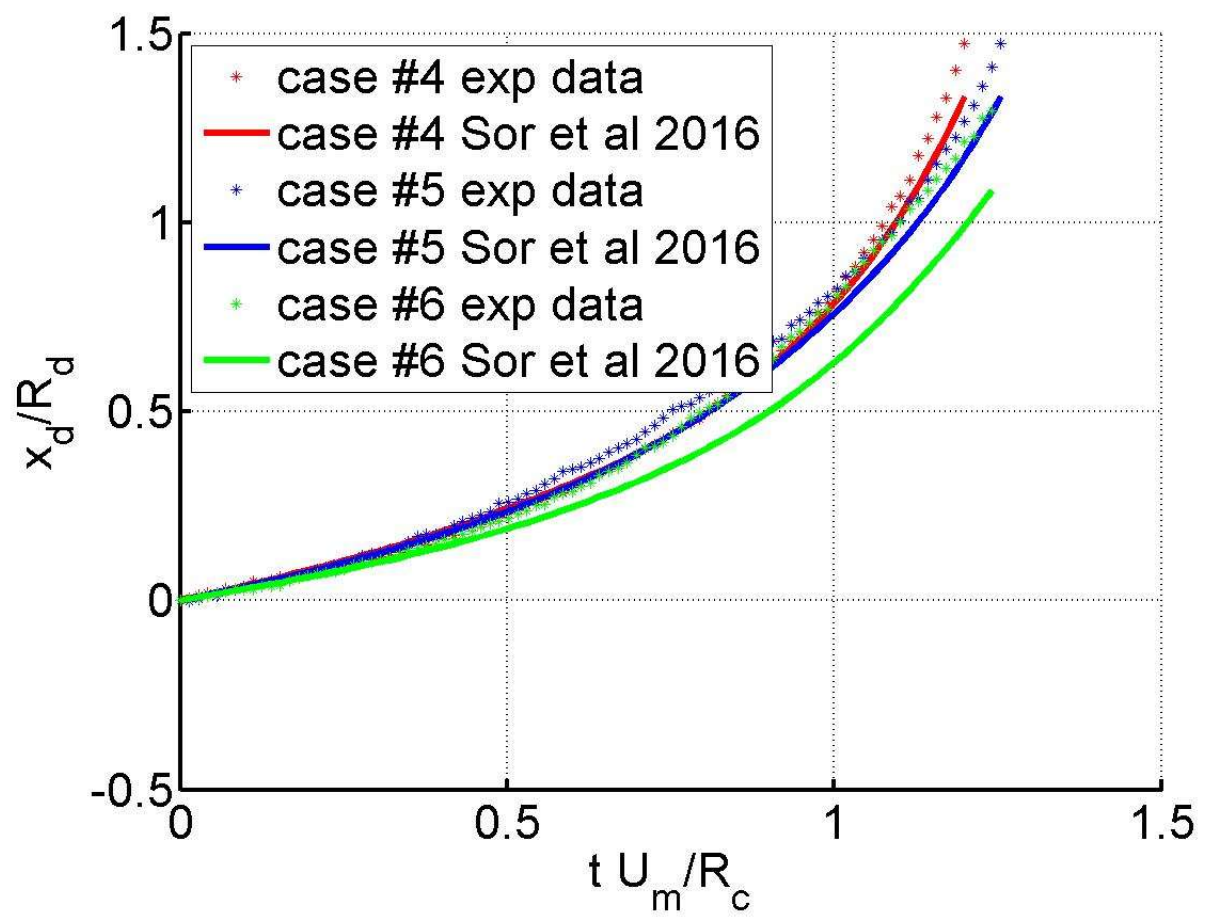

Figure 5.3.8. Comparison of the recorded droplet's horizontal position for cases \#4, \#5, and \#6 with those obtained by integrating the theoretical model. 


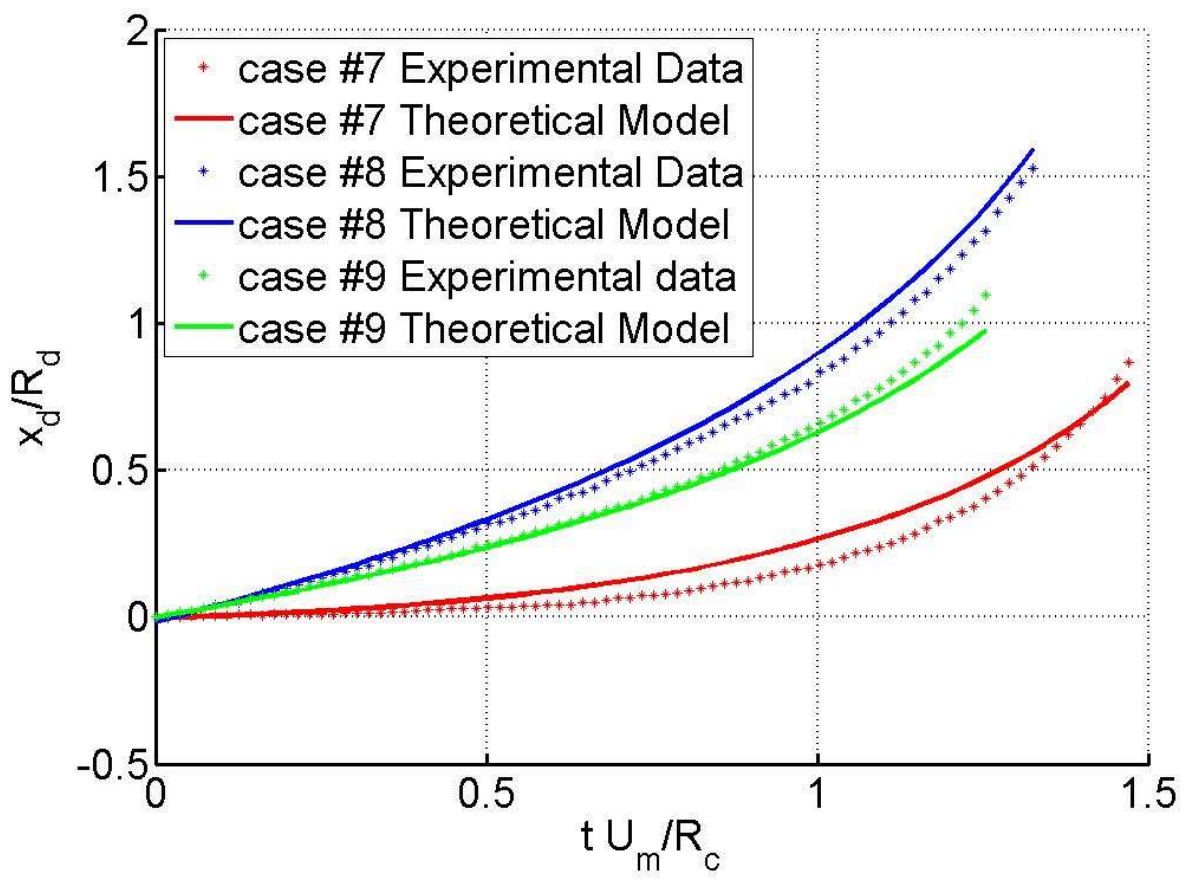

Figure 5.3.9. Comparison of the recorded droplet's horizontal position for cases \#7, \#8, and $\# 9$ with those obtained by integrating the theoretical model.

It could be observed in figures 5.3.7 and 5.3.8 that the discrepancies between the experimental data and the results provided by the theoretical are larger in cases \#3 and \#6 that correspond to FOV \#3, see figure 5.1.1. That is: the model results tend to deteriorate when moving away from the airfoil leading edge region. However, this is not what happens in the case of the higher airfoil velocity, see figure 5.3.9. In this case, the theoretical model provides results with the same level of discrepancy no matter what FOV is considered.

In a sense, these aerodynamics interference effects depend on the liquid water content (LWC) of the cloud that the actual airfoil is immersed into. In heavy rainfall, LWC is of the order of $32 \mathrm{gr} / \mathrm{m}^{3}$ with droplet diameters around $6 \mathrm{~mm}$. These figures can go down to LWC of about $1 \mathrm{gr} / \mathrm{m}^{3}$ with droplet diameters of $1 \mathrm{~mm}$. However, the experimental setup used in this work dealt with trains of droplets not with clouds of droplets, which means that interference effects in both situations are markedly different. For example, the separation between consecutive droplets in the experiment is smaller than the typical separation in the case of heavy rainfall (LWC of about $32 \mathrm{gr} / \mathrm{m}^{3}$ ). This means that the discrepancies observed between experimental and theoretical data in figures 5.3.7, 5.3.8, and 5.3.9 (that are caused by aerodynamics interference) might be somewhat smaller in an actual flying situation. 


\subsection{CONCLUSIONS.}

An experimental study has been presented on the subject of water droplet deformation in the vicinity of the shoulder region of an incoming airfoil. Nine different cases have been considered that correspond to three airfoil velocities $(50 \mathrm{~m} / \mathrm{s}, 70 \mathrm{~m} / \mathrm{s}$, and $90 \mathrm{~m} / \mathrm{s})$ and three different fields of vision located close to the shoulder region of the airfoil. Possibly, the most relevant aspect of the results obtained is that, contrary to what happens in the airfoil leading edge region, the droplets moving in the shoulder region tend to tilt significantly towards the direction of the incoming airfoil.

This tilting tends to cause aerodynamics interference between consecutive droplets. The consequence of this type of behavior is that existing droplet trajectory and deformation models that make reasonably accurate predictions in the airfoil leading edge region tend to generate less accurate when applied to droplets moving in the airfoil shoulder region. However, the LWC of actual heavy rainfall leads to droplet separation distances that are larger than those obtained in the experiment; so the question that subsides is whether the theoretical model developed might still be reasonably valid for engineering applications even in the shoulder region of an airfoil.

In any case, this issue raises some questions regarding the development of engineering methods and tools for prediction of rainfall or icing conditions effects on aircraft. In particular, two of these questions are:

a) Would it be possible, owing to the markedly different types of behavior observed, to develop a single generic model that accounts for droplet deformation and trajectory all around the airfoil?

b) Does it still make sense to develop methods and tools that are based on the behavior of the individual droplets?

Then, in this context, the present experimental study could be regarded as an approach that has contributed to pose some questions that might be of interest when addressing the development of future engineering methods and tools in this field. 


\section{CONCLUSIONS.}

A theoretical model has been presented that predicts droplet trajectory and deformation in the vicinity of an incoming airfoil. The model is of interest both to gain a better understanding of droplet trajectories in some flows of aeronautics interest, and to provide an alternate formulation to researchers that develop numerical flow solvers in the field of icing conditions simulations. The model has been tested and calibrated using a series of dedicated experimental tests in a rotating arm facility. The novelty of the study consists on the fact that it accounts for the actual flow acceleration profile that the droplet senses as the airfoil closes on it. This is contrast to other more basic-physics oriented studies in which shock tube type acceleration profiles are considered. The conclusions that have been obtained are, basically, three.

First, it is worth mentioning that a relatively simple model based on droplet dynamics and deformation (three ordinary differential equations) is sufficient to predict with reasonable accuracy droplet trajectory in the conditions that have been considered. The model can be said to have a "global" character because it implements force formulations that are "global" themselves. For example, the pressure force does not depend on the actual local coordinate on the droplet surface but it is modeled after a "global" coefficient. This suggests that the two problems of: a) the onset and further development of flow instabilities leading to droplet deformation and eventual break-up, and b) computation of droplet trajectory, are loosely coupled only. That is, once the conditions that define the basic type of deformation and break-up are set, the droplet trajectory can be modeled somewhat independently. For example, in the parametric range of cases that have been considered, assuming that the droplet deforms following the shape of a spheroid appears to be enough for trajectory prediction purposes.

The second question to be noted is that in the cases that have been addressed in the experimental campaign, implementation of an unsteady term in the aerodynamics drag model (the so-called acceleration parameter) is essential to allow for model accuracy. The issue of separating the drag into quasi-steady and unsteady parts (each one with its own modelization) is somewhat controversial and no claim is made in this thesis regarding its generalization. The only thing that can be said is that for the range of parameters under consideration in the present experimental campaign, the unsteady part of the drag coefficient was critically needed to compute trajectories that compared well to the observed trajectories. Regarding the unsteady drag model itself, the main differences between the model proposed in this article and previous results put forward by other researchers are two:

a) In the present model the acceleration parameter includes, also, the time dependent droplet dimension in the slip velocity-wise direction. This parameter was not considered in formulations described previously in the literature. In this sense, it could be said that its inclusion here makes sense because of the fact that droplets 
deformed significantly in these rotating arm experiments. It is to be noted that in other experimental studies researchers sometimes used droplets manufactured on materials (plastic type) that did not allow for large deformations. On the Fluid Mechanics side, the reason for the implementation of the unsteady drag term is based on the fact that in an accelerating (or decelerating flow) the size of the recirculation region downstream of the droplet changes continuously; and the dynamics of this change affect the evolution of the drag. Then, in this regard, it is sensible to modify the acceleration parameter so as to account for information on droplet continuous deformation in the slip velocity-wise direction that also affects the dynamics of the recirculation region.

b) The coefficient that multiplies the modified acceleration parameter in the present model is 9. In other studies dealing with different experimental setups, researchers found that this coefficient was dependent, also, on some other parameter(s) of the problem. However, in the present study it was found that for the range of parameters under consideration, the coefficient was always 9.

The third question is related to the deformation modelization. A new equation is proposed for the deformation of droplets based on a spring-mass analogy similar to Clark's and the DDB models. All three models include only forces due to surface tension, viscosity and pressure. A comparison has been made between the three models and experimental data of water droplets approaching the leading edge of an airfoil along the stagnation line. It can be stated about the results:

1. The new deformation equation predicts accurately the deformation of droplets of radius between $200 \mu \mathrm{m}$ and $700 \mu \mathrm{m}$ being distorted by an increasing exponential velocity flow field as experienced by a droplet approaching the leading edge of an airfoil.

2. The surface tension force model proposed, based on an oblate spheroid deformation, works well without the simplification used in the DDB model.

3. The assumptions about the pressure forces, estimated to be proportional to the dynamic pressure and the projected area of the non-distorted droplet, seems to work well, being the proportionality coefficient provided by experimental data 0.93 .

4. Viscosity force seems to have little influence in the final results, so the assumption of a pure extensional flow appears to be accurate enough.

Finally, it should be said that the previous conclusions only apply for the cases that consider the flow acceleration profile generated by an incoming airfoil. This means that 
these conclusions cannot be extrapolated to other situations. A systematic study on the influence that functionally different acceleration profiles may have on droplet deformation and droplet trajectory is out of the scope of the present study.

Then, an experimental study has been presented on the subject of water droplet deformation in the vicinity of the shoulder region of an incoming airfoil. Nine different cases have been considered that correspond to three airfoil velocities $(50 \mathrm{~m} / \mathrm{s}, 70 \mathrm{~m} / \mathrm{s}$, and $90 \mathrm{~m} / \mathrm{s}$ ) and three different fields of vision located close to the shoulder region of the airfoil. Possibly, the most relevant aspect of the results obtained is that, contrary to what happens in the airfoil leading edge region, the droplets moving in the shoulder region tend to tilt significantly towards the direction of the incoming airfoil.

This tilting tends to cause aerodynamics interference between consecutive droplets. The consequence of this type of behavior is that existing droplet trajectory and deformation models that make reasonably accurate predictions in the airfoil leading edge region tend to generate less accurate when applied to droplets moving in the airfoil shoulder region. However, the LWC of actual heavy rainfall leads to droplet separation distances that are larger than those obtained in the experiment; so the question that subsides is whether the theoretical model developed might still be reasonably valid for engineering applications even in the shoulder region of an airfoil.

In any case, this issue raises some questions regarding the development of engineering methods and tools for prediction of rainfall or icing conditions effects on aircraft. In particular, two of these questions are:

a) Would it be possible, owing to the markedly different types of behavior observed, to develop a single generic model that accounts for droplet deformation and trajectory all around the airfoil?

b) Does it still make sense to develop methods and tools that are based on the behavior of the individual droplets?

Then, in this context, the present experimental study could be regarded as an approach that has contributed to pose some questions that might be of interest when addressing the development of future engineering methods and tools in this field. 


\section{BIBLIOGRAPHY.}

Aggarwal, S. K., Peng, F., 1995, “A Review of Droplet Dynamics and Vaporization Modeling for Engineering Calculations", J. Eng. Gas Turbines and Power, 117, 453-461.

Auton, T. R., Hunt, J. C. R., Prud'homme, M., 1988, "The force exerted on a body in inviscid unsteady non-uniform rotational flow”, J. Fluid Mech. 197, 241-257.

Bragg, M. B., 1982, “A Similarity Analysis of the Droplet Trajectory Equation”, AIAA J., 20 (12), 1681-1686.

Cao, Y., Wu, Z., Xu, Z., 2014, "Effects of rainfall on aircraft aerodynamic", Progress in Aerospace Sciences 71, 85-127.

Clark, M. M., 1988, "Drop Breakup in a Turbulent Flow- I, Conceptual and Modeling Considerations", Chemical Engineering Science, Vol. 43, No. 3, pp. 671-679.

Cober, S. G., Isaac, G., Shah, A. D., Jeck, R., 2003, "Defining characteristic cloud droplet spectra from in-situ measurements", 41st Aerospace Sciences Meeting and Exhibit, Aerospace Sciences Meetings, volume 2003-561.

Crowe, C. T., Nicholls, J. A., Morrison, R. B., 1963, "Drag coefficients of inert and burning particles accelerating in gas streams", In Symposium (International) on Combustion, volume 9, 395-406. Elsevier.

Feo, A. Vargas, M., Sor, S., 2011, "Rotating Rig Development for Droplet Deformation/Breakup and Impact Induced by Aerodynamic Surfaces", SAE Paper Number 2011-38-0087.

Flock, A. K., Guildenbecher, D. R., Chen, J., Sojka, P. E., Bauer, H. J., 2012, "Experimental statistics of droplet trajectory and air flow during aerodynamic fragmentation of liquid drops", Int. J. of Multiph. Flow 47, 37-49.

Garcia-Magariño, A., Sor, S., Velazquez, A., 2015, "Experimental characterization of water droplet deformation and breakup in the vicinity of a moving airfoil", Aerosp. Sci. Technol., 45, 490-500.

Hansman, R.J., Craig, A. P., 1987, "Low Reynolds number tests of NACA 64-210 NACA 0012 and Worthmann FX67-K170 airfoils in rain", Journal of Aircraft 24, 559-566. 
Hsiang, L. P., Faeth, G. M., 1995, "Drop deformation and breakup due to shock wave and steady disturbances", Int. J. Multiph. Flow, 21 (4), 545-560.

Hwang, S. S., Liu, Z., Reitz, R. D., 1996, "Breakup mechanisms and drag coefficients of high-speed vaporizing liquid drops", Atomization and Sprays, 6 (3).

Ibrahim, E. A., Yang, H. Q., Przekwas, A. J., 1993, "Modeling of Spray Droplets Deformations and Breakup", AIAA Journal of Propulsion and Power, Vol. 9, No. 4, pp. 652-654.

Igra, O., Takayama, K., 1993, "Shock tube study of the drag coefficient of a sphere in a non-stationary flow", Proc. R. Soc. A 442, 231-247.

Iuliano, E., Mingione, G., Petrosino, F., and Hervy, F., 2011, "Eulerian modeling of large droplet physics toward realistic aircraft icing simulation", J. Aircraft, 48, 1621-1632.

Jenkins, D. C., Booker, J. D., 1965, "The time required for hig speed airstreams to disintegrate water drops", Current Papers 827, Ministry of Aviation- Aeronautical research council.

Jourdan, G., Houas, L., Igra, O., Estivalezes, J. L., Devals, C., Meshkov, E. E., 2007, "Drag coefficient of a sphere in a non-stationary flow: new results", Proc. R. Soc. A 463, $3323-3345$.

Kabaliuk, N, Jermy, M. C., Williams, E., Laber, T. L., Taylor, M. C., 2014, "Experimental validation of a numerical model for predicting the trajectory of blood drops in typical crime scene conditions, including droplet deformation and breakup, with a study of the effect of indoor air currents and wind on typical spatter drop trajectories", Forensic Sci. Int. 245, 107-120.

Karanfilian, S. K., Kotas, T. J., 1978, "Drag on a sphere in unsteady motion in a liquid at rest", Journal of Fluid Mechanics, 87(01), 85-96.

Krezel, J., Mc Nichols, W., Prete, J., 2008, "Causality analysis for aviation weather hazards", AIAA 2008- 8914. 26th Congress of the International Council of Aeronautical Sciences (ICAS) Anchorage.

Krzeczkowski, S. A., 1980, "Measurements of Liquid Droplet Disintegration Mechanisms", International Journal of Multiphase Flow, Vol. 6, No. 1, pp. 227-239.

Lampton, A., Valasek, J., 2012, "Prediction of icing effects on the lateral/directional stability and control of light airplanes”, Aerosp. Sci. Technol. 23, 305-311. 
Lee, C. S., Kim, H. J., Park, S. W., 2004, “Atomization characteristics and prediction accuracies of hybrid break-up models for a gasoline direct injection spray", Proceedings of the Institution of Mechanical Engineers, Part D, Journal of Automobile Engineering, 218(9), 1041-1053.

Liu, A. B., Mather, D., Reitz, R. D., 1993, "Modeling the effects of drop drag and breakup on fuel sprays", Technical report, DTIC Document.

Liu, Z., Reitz, R. D, 1997, “An analysis of the distortion and breakup mechanisms of high speed liquid drops. International journal of multiphase flow", 23(4), 631-650. IF:0.726.

Luxford, G., Hammond, D. W., Ivey, P., 2005, "Modelling, imaging and measurement of distortion, drag and break-up of aircraft-icing", 43rd AIAA Aerospace Sciences Meeting and Exhibit, volume 75, Reno, Nevada, January

Maxeya, M. R., Riley, J. J., 1983, 'Equation of motion for a small rigid sphere in a nonuniform flow", Phys. Fluids 26 (4), 883-889.

Mingione, G, Guffond, D., Tropea, C., 2011 "Extice: Extreme icing environment", SAE International 2011-38-0063.

Mohebil, M. M, Evans, J. R. G., 2005, “The trajectory of ink-jet droplets: Modeling and experiment”, Chem. Eng. Sci. 60, 3469-3476.

Odar, F., Hamilton, W. S., 1964, "Forces on a sphere accelerating in a viscous fluid", Journal of Fluid Mechanics, 18(02), 302-314.

O’Rourke, P. J., Amsden, A. A., 1987, “The TAB Method for Numerical Calculations of Spray Droplet Breakup” Society of Automotive Engineers Paper 87-2089.

Ortiz, C., Joseph, D. D., Beavers, G. S., 2004, “Acceleration of a liquid dropsuddenly exposed to a high-speed airstream", International journal of multiphase flow, 30(2), 217 224. IF:1.383. Q1.

Otsu, N., 1979, "A threshold Selection Method from Gray-Level Histograms", IEEE Transactions on systems, man, and cybernetics, Vol smc-9, $\mathrm{N}^{\circ} 1$.

Papadakis, M., Wong, S. C., Rachman, A., Hung, K. E., Vu, G. T., Bidwell, C. S., 2007, "Large and Small Droplet Impingement Data on Airfoils and Two Simulated Ice Shapes", NASA/TM-2007-213959. 
Park, S W., Lee, C. S., 2004, "Investigation of atomization and evaporation characteristics of high-pressure injection diesel spray using kelvin-helmholtz instability/ droplet deformation and break-up competition model", Proceedings of the Institution of Mechanical Engineers, Part D, Journal of Automobile Engineering, 218(7), 767777.DOI: 10.1081 / SS-120039314.

Park, J. H., Yoon, Y., Hwang, S.S., 2002, "Improved TAB model for prediction of spray droplet deformation and breakup", Atomization and Sprays, 12(4). IF:0.882.

Petera, J., Rooney, D., Weatherley, L. R., 1998, "Particle and droplet trajectories in a nonlinear electrical field”, Chem. Eng. Sci. 53 (22), 3781-3792.

Petera, J., Weatherley, L. R., Hume, A. P., Gawrysiak, T., 2007, “A finite element algorithm for particle/droplet trajectory tracking, tested in a liquid-liquid system in the presence of an external electric field", Comput. Chem. Eng. 31, 1369-1388.

Pham, T. L., Heister, S. D., 2002, "Spray modeling using lagrangian droplet tracking in a homogeneous flow model", Atomization and Sprays, 12(5).

Rendall, T. C. S., Allen C. B., 2014, "Finite-volume droplet trajectories for icing simulation”, Int. J. Multiph. Flow 58, 185-194.

Reehorst, A. L., Ibrahim, M., 1995, "Investigation of Water Droplet Trajectories Within the NASA Icing Research Tunnel”, NASA/TM 107023.

Reehorst, A., Chung, J. , Potapezu, K M., 2000, "Study of icing effects on performance and control ability on aircraft", Journal of Aircraft 37, 253-259.

Saeed, F, Brette, C., Fregeau, M., Trifu, O., Paraschivoiu, I., 2005, “A Three-Dimensional Water Droplet Trajectory and Impingement Analysis Program", in: $23^{\text {rd }}$ AIAA Applied Aerodynamics Conference, Toronto, Ontario, Canada, AIAA 2005-4838.

Schmehl, R., 2002, “Advanced Modeling of Droplet Deformation and Breakup for CFD Analysis of Mixture Preparation", 18th Annual Conference on Liquid Atomization and Spray Systems (ILASS-Europe), Zürich, Schweiz, 9-11 September.

Simpkins, P. G., Bales, E. L., 1972, "Water-drop response to sudden accelerations", Journal of Fluid Mechanics, 55(04), 629-639.

Sinha, A., Prakash, R. S., Mohan, A. M., Ravikrishna, R. V., 2015, "Airblast spray in crossflow-Structure, trajectory and droplet sizing”, Int. J. Multiph. Flow 72, 97-111. 
Sor, S., García-Magariño, A., 2011, "PIV Measurements on Airfoil Model in Rotating Arm”, AE-TNO-4420-091- INTA-11.

Sor, S., García-Magariño, A., 2012, "Influence of illumination on shadow image analysis with SITEA", AE-TNO-4420-039- INTA-12.

Sor, S., Garcia-Magariño, A., 2015, "Modeling of droplet deformation near the leading edge of an airfoil”, J. Aircraft, 52, 1838-1846.

Sor, S., García-Magariño, A., Morote, J., 2012, "Experimental determination and data processing of trajectories, deformation and breakup of water droplets impinging on airfoils", Conference on Modeling Fluid Flow, Budapest.

Sor, S., García-Magariño, A., Velázquez, A., 2016, "Model to predict water droplet trajectories in the flow past an airfoil", Aerosp. Sci. Technol., 58, 26-35.

Taskiran, O. O., Ergeneman, M., 2014, "Trajectory based droplet collision model for spray modeling”, Fuel 115, 896-900.

Taylor, G. I., 1963, "The Shape and Acceleration of a Drop in a High Speed Air Stream”, volume III of The Scientific Papers of G. I. Taylor. University Press, Cambridge.

Temkin, S., Mehta, H. K., 1982, "Droplet drag in an accelerating and decelerating flow", Journal of Fluid Mechanics, 116, 297-313.

Theofanous, T. G., 2011, "Aerobreakup of Newtonian and viscoelastic fluids", Annu. Rev. Fluid Mech. 43, 661-690.

Theofanous, T. G., Li, G. J., 2008, "On the physics of aerobreakup", Phys. Fluids. 20, 052103 .

Thomas, P. R., Kurt, B., Willian, R., 2003, "Iced aircraft flight data for flight simulator validation", NASA Tm / $2003-212114$.

TSI, 2002, “TSI Aerometrics MDG-100 Monosize Droplet Generator Instruction Manual," P/N 1990870, June.

Vargas, M., Feo, A., 2010, "Experimental Observations on the deformation and Breakup of Water Droplets near the leading Edge of an Airfoil”, NASA/TM-2011-216946, AIAA Paper 2010-7670, August. 
Vargas, M., Feo, A., 2011, "Deformation and Breakup of Water Droplets near an Airfoil Leading Edge", Journal of Aircraft, 48,1749-1765. IF:0.538. Q3.

Vargas, M., 2012, "Droplet Deformation Prediction with the Droplet Deformation and Breakup Model (DDB)", AIAA Atmospheric and Space Environments Conference, AIAA 2012-3131, New Orleans.

Vargas, M., Sor, S., Garcia Magariño, A., 2012, "Mechanism of Water droplet Breakup Near the leading Edge of an Airfoil", AIAA Atmospheric and Space Environments Conference, AIAA 2012-3129, New Orleans.

Vargas, M., Sor, S., Garcia Magariño, A., 2013,"Drag Coefficient of Water Droplets Approaching the Leading Edge of an Airfoil", AIAA Atmospheric and Space Environments Conference, AIAA 2013-3054, San Diego.

Yang, Z., Krieger, K., Lunt, T., Brochard, F, Briancon, J. L., Neu, R., Dux, R., Janzer, A., Potzel, S., Pütterich, T., 2013, “3D trajectories re-construction of droplets ejected in controlled tungsten melting studies in ASDEX Upgrade”, J. Nucl. Mater. 438, 5846-5851.

Zarrabeitia, L. A., Qureshi, F. Z, Aruliah, D. A., 2015, "Stereo Reconstruction of Droplet Flight Trajectories”, in IEEE Trans. Pattern Anal. Mach. Intel., 37 (4), 847-861,doi: 10.1109/TPAMI.2014.2353638.

Zhang, R., Cao, Y., 2010, "Study of aerodynamics characteristics on an airfoil in rain," Journal of Aerospace Power, 25, 2064-2069.

Zhang, L., Yang, C., Mao, Z.S., 2008, “Unsteady motion of a single bubble in highly viscous liquid and empirical correlation of drag coefficient”, Chem. Eng. Sci. 63, 20992106. 